\title{
Online consumer-to-consumer communication networks : an economic exploration of their formation and value
}

Citation for published version (APA):

Harmsen - van Hout, M. J. W. (2009). Online consumer-to-consumer communication networks : an economic exploration of their formation and value. [Doctoral Thesis, Maastricht University]. Datawyse / Universitaire Pers Maastricht. https://doi.org/10.26481/dis.20090319mh

Document status and date:

Published: 01/01/2009

DOI:

10.26481/dis.20090319mh

Document Version:

Publisher's PDF, also known as Version of record

Please check the document version of this publication:

- A submitted manuscript is the version of the article upon submission and before peer-review. There can be important differences between the submitted version and the official published version of record.

People interested in the research are advised to contact the author for the final version of the publication, or visit the DOI to the publisher's website.

- The final author version and the galley proof are versions of the publication after peer review.

- The final published version features the final layout of the paper including the volume, issue and page numbers.

Link to publication

\footnotetext{
General rights rights.

- You may freely distribute the URL identifying the publication in the public portal. please follow below link for the End User Agreement:

www.umlib.nl/taverne-license

Take down policy

If you believe that this document breaches copyright please contact us at:

repository@maastrichtuniversity.nl

providing details and we will investigate your claim.
}

Copyright and moral rights for the publications made accessible in the public portal are retained by the authors and/or other copyright owners and it is a condition of accessing publications that users recognise and abide by the legal requirements associated with these

- Users may download and print one copy of any publication from the public portal for the purpose of private study or research.

- You may not further distribute the material or use it for any profit-making activity or commercial gain

If the publication is distributed under the terms of Article $25 \mathrm{fa}$ of the Dutch Copyright Act, indicated by the "Taverne" license above, 
Online Consumer-to-Consumer Communication Networks

An Economic Exploration of their Formation and Value

Marjolein J.W. Harmsen - van Hout 
(c) 2009, Marjolein J.W. Harmsen - van Hout, Simpelveld

All rights reserved. No part of this publication may be reprinted or utilized in any form or by any electronic, mechanical, or other means, now known or hereafter invented, including photocopying and recording, or in any information storage or retrieval system, without written permission from the copyright owner.

Published by Universitaire Pers Maastricht

ISBN 978-90-5278-814-2

Cover design: Michiel van Hout / www.michielvanhout.nl

Printed by Datawyse Maastricht 


\section{Online Consumer-to-Consumer Communication Networks}

An Economic Exploration of their Formation and Value

\section{PROEFSCHRIFT}

ter verkrijging van de graad van doctor aan de Universiteit Maastricht,

op gezag van de Rector Magnificus, prof. mr. G.P.M.F. Mols,

volgens het besluit van het College van Decanen,

in het openbaar te verdedigen

op donderdag 19 maart 2009 om 16.00 uur

door

Maria Juliëtte Wilhelmina Harmsen - van Hout 


\section{Promotores}

Prof. dr. ir. Benedict G.C. Dellaert (Erasmus Universiteit Rotterdam)

Prof. dr. P. Jean-Jacques Herings

\section{Beoordelingscommissie}

Prof. dr. Arno Riedl (voorzitter)

Dr. Els Breugelmans

Prof. dr. Dolf J.J. Talman (Universiteit van Tilburg) 


\section{Contents}

List of Tables vii

List of Figures $\quad$ ix

Preface $\quad$ xi

1 Introduction 1

1.1 Online Consumer-to-Consumer Communication Networks . . 1

1.2 An Economic Exploration of their Formation and Value . . . 3

1.3 Methods and Outline . . . . . . . . . . . . . . 4

1.3.1 The Strategic Aspect . . . . . . . . . . . . . 4

1.3.2 The Behavioral Aspect . . . . . . . . . . . . . 6

1.3 .3 Outline ................... 7

2 The Strategic Aspect: A Game-Theoretical Model 9

2.1 Introduction . . . . . . . . . . . . . . . . . 10

2.2 Social Value . . . . . . . . . . . . . . . . . . . 12 
2.2 .1 Model . . . . . . . . . . . . . . . 13

2.2 .2 Results . . . . . . . . . . . . . 16

2.3 Informational as well as Social Value . . . . . . . . . . . 23

2.3 .1 Model . . . . . . . . . . . . . . . . . . . . . 24

2.3 .2 Results . . . . . . . . . . . . . . 27

2.4 Welfare ........................ 36

2.4.1 Best Pairwise Stable Structures . . . . . . . . . . . 36

2.4.2 Worst Pairwise Stable Structures . . . . . . . . . . 38

2.4.3 Informational versus Social Orientation $\ldots \ldots$. . . . 40

2.5 Discussion . . . . . . . . . . . . . . . 43

3 The Strategic Aspect: A Simulation Approach 45

3.1 Introduction . . . . . . . . . . . . . . . . 46

3.2 Model . . . . . . . . . . . . . . . . . . . . . 47

3.3 Method . . . . . . . . . . . . . . . . . . 48

3.4 Definitions . . . . . . . . . . . . . . . . 50

3.4.1 Valuation Patterns . . . . . . . . . . . . . 50

3.4 .2 Structure Measures . . . . . . . . . . . . . . 52

3.5 Results........................ 53

3.5 .1 Convergence . . . . . . . . . . . . 53

3.5 .2 Homogeneity . . . . . . . . . . . . . . . 53

3.5.3 Homogeneity with Expert . . . . . . . . . . 56 
3.5.4 Expertise Heterogeneity . . . . . . . . . . . . 65

3.5.5 Expertise Heterogeneity with Expert . . . . . . . . 70

3.6 Discussion . . . . . . . . . . . . . . 76

3.6.1 Measurement . . . . . . . . . . . . 76

3.6 .2 Management . . . . . . . . . . . . 78

3.6 .3 Further Research . . . . . . . . . . . . . . . 78

\section{The Behavioral Aspect: An Experimental Investigation 81}

4.1 Introduction . . . . . . . . . . . . . . . . 82

4.2 Theoretical Framework . . . . . . . . . . . . . . . 84

4.2.1 Prior Utility Models of Network Formation Decisions . 85

4.2 .2 Hypotheses . . . . . . . . . . . . . . . 86

4.3 Methods .......................... 94

4.3.1 Experimental Design . . . . . . . . . . . . . . . . 94

4.3.2 Mixed Logit Estimations . . . . . . . . . . . . . . . . 97

4.4 Results. . . . . . . . . . . . . . . . . . . 100

4.4.1 Some Illustrative Descriptive Results . . . . . . . . . 100

4.4 .2 Mixed Logit Results . . . . . . . . . . . . . . 102

4.5 Discussion . . . . . . . . . . . . . . . . . 108

4.5 .1 Results . . . . . . . . . . . . . 108

4.5 .2 Implications . . . . . . . . . . . . . . . . . . 109 
5.1 OCCNs: An Economic Exploration of their Formation and Value ........................ . . 111

5.2 Managerial Implications . . . . . . . . . . . . . . . . 113

5.3 Further Research . . . . . . . . . . . . . . . . . . . . . 114

5.3.1 The Strategic Aspect . . . . . . . . . . . . . . . 114

5.3.2 The Behavioral Aspect . . . . . . . . . . . . 115

$\begin{array}{ll}\text { A Instructions } & 117\end{array}$

A.1 Social Tradeoff? NO Value Transferability? NO YES . . 117

A.2 Social Tradeoff? YES Value Transferability? NO YES . 118

A.3 Example . . . . . . . . . . . . . . . . 120

B Choice Problems 123

$\begin{array}{ll}\text { C Descriptive Results } & 129\end{array}$

$\begin{array}{ll}\text { Bibliography } & 131\end{array}$

Nederlandse Samenvatting (Summary in Dutch) 139

$\begin{array}{ll}\text { Curriculum Vitae } & 145\end{array}$ 


\section{List of Tables}

1.1 Examples of OCCNs . . . . . . . . . . . . 3

2.1 Pairwise Stable Structures With Informational Value . . . . . 34

3.1 Densities for Homogeneity . . . . . . . . . . . . . . . . . 54

3.2 Example of Structures for Homogeneity . . . . . . . . . 55

3.3 Welfare for Homogeneity . . . . . . . . . . . . . 55

3.4 Densities for Homogeneity with Expert . . . . . . . . . . 62

3.5 Centralities for Homogeneity with Expert . . . . . . . . . 63

3.6 Example of Structures for Homogeneity with Expert . . . . . 64

3.7 Welfare for Homogeneity with Expert . . . . . . . . . . . 64

3.8 Densities for Expertise Heterogeneity . . . . . . . . . . . . 67

3.9 Centralities for Expertise Heterogeneity . . . . . . . . . . 68

3.10 Example of Structures for Expertise Heterogeneity . . . . . . 69

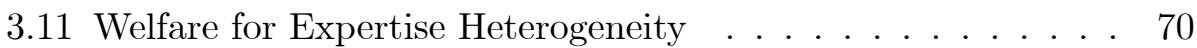

3.12 Payoffs for Expertise Heterogeneity . . . . . . . . . . . . 71 
3.13 Densities for Expertise Heterogeneity with Expert $\ldots . .72$

3.14 Centralities for Expertise Heterogeneity with Expert . . . . 73

3.15 Example of Structures for Expertise Heterogeneity with Expert 74

3.16 Welfare for Expertise Heterogeneity with Expert . . . . . . 74

3.17 Payoffs for Expertise Heterogeneity with Expert . . . . . . 75

4.1 Experimental Design . . . . . . . . . . . . . . . . . 95

4.2 Descriptive Results Choice Problem 5 . . . . . . . . . . . 101

4.3 Mixed Logit Estimations Without Payoff Information . . . . 103

4.4 Mixed Logit Estimations Including Payoff Information . . . . 106

4.5 Summary Experimental Results . . . . . . . . . . . . . . . 108

B.1 Practice Rounds . . . . . . . . . . . . . . . . . . . . . . 124

B.2 Choice Problems $1-3 \ldots \ldots \ldots \ldots$

B.3 Choice Problems $4-6 \ldots \ldots \ldots$

B.4 Payoffs Choice Problems . . . . . . . . . . . . . . 127 


\section{List of Figures}

1.1 Dissertation Summary . . . . . . . . . . . . . . 8

2.1 A Non-Regular Pairwise Stable Structure (Without Informational Value) ...................... 22

2.2 A Multiple-Component Pairwise Stable Structure (Without Informational Value $) \ldots \ldots . \ldots . \ldots . . \ldots 23$

2.3 A "Small World" Pairwise Stable Structure (Without Infor-

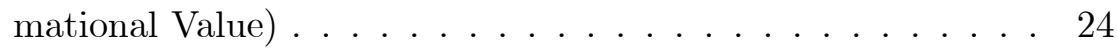

2.4 Impact of $\alpha$ on Welfare Illustrated for $n=10 \ldots \ldots 4$

3.1 A Non-Star Pairwise Stable Structure in the Expert Case . . 61

4.1 Example . . . . . . . . . . . . . . . . 85

4.2 Hypothesized Model . . . . . . . . . . . . . . . . . . 94

B.1 Illustration Payoff Information . . . . . . . . . . . . 123 


\section{Preface}

The most difficult thing about doing research is to stop doing it. In particular this holds for a specific piece of work like an article or this dissertation. There must be a moment on which it is declared finished. This is what I do now by writing this preface. To reduce the difficulty of this task, I intend to keep on doing research after my $\mathrm{PhD}$, which makes sure that it is not my ultimate expression. Still, this fictional finish is a good opportunity to show something of what I was doing during the last years and moreover to thank everybody who supports me.

This dissertation reveals some insights about our networked society. Everybody deals with communication links in everyday life, everybody is a consumer, and almost everybody has encountered the rich environment of the internet. This suggests that almost everyone likes to read this book about online consumer-to-consumer communication networks. That this is not the case is certainly due to time constraints (see Chapter 2 of this thesis). I sincerely hope that any other reasons do not exist, for I have attempted to write something sufficiently noteworthy and novel about this relevant topic.

My sincere thanks go to everybody who did invest time and effort in this dissertation. In particular, I would like to mention my supervisors Benedict Dellaert and Jean-Jacques Herings who merged two different research traditions in this project and provided a large informational value. Furthermore, I would like to thank the members of the dissertation committee, Arno Riedl, Els Breugelmans, and Dolf Talman, as well as the participants in the expe- 
riment or one of its pilots, for their informational value. Also thanks to all my teachers, colleagues and office mates at the FEBA, who supplied a mix of informational and social value. Moreover, I would like to thank my family, friends, and especially my husband for the large social (and sometimes informational!) value they gave me. Finally, it is right and just to thank God who created and maintains all value. 


\section{Chapter 1}

\section{Introduction}

This chapter introduces the dissertation's topic (Section 1.1), perspective (Section 1.2), and methods (Section 1.3).

\subsection{Online Consumer-to-Consumer Communication Networks}

The importance of consumers is increasingly recognized in today's society. During the last century, the predominant business philosophy evolved from the production concept, perceiving consumers as taken-for-granted buyers of a firm's product, via the selling concept, perceiving consumers as potential buyers of a firm's product who have to be convinced, to the marketing concept, perceiving consumers as individuals with needs that a firm's product should satisfy. More recently, consumers are even often perceived as active partners in a firm's production process, since they are specialized in their own preferences and after buying in using the product (Von Hippel 1998).

A prevailing way in which consumers can perform this active role is by communicating with other consumers. By exchanging experiences with a 
product, consumers do not only help themselves and each other, but they simultaneously help the firm if it is sufficiently knowledge-oriented and flexible (Prahalad and Ramaswamy 2000). Evidently, by helping each other consumers reduce the firm's product support costs. Additionally, they provide the firm with insider feedback that is useful for product improvement and they develop and promote a more favorable attitude towards the product (Wiertz 2005). Thus, consumer and firm welfare are highly related in this context.

It has been acknowledged in the social sciences (Wellman and Berkowitz 1988) and particularly in marketing (Iacobucci 1996) that human behavior should be studied from a structure or network perspective, because it is affected not only by individual entities such as firm, consumer or household, or even dyads such as buyer-seller or consumer-consumer, but also by the entire pattern of relationships among multiple actors. Therefore, in this dissertation we deal with the relevant topic of consumer-to-consumer communication in the form of networks.

A growingly popular version of such a network is the online consumerto-consumer communication network (OCCN). Online, consumers are more flexible to choose their communication partners based on shared interest than offline, for they are less constrained by existing acquaintance structures. Also, it is much easier for a firm to gain insight from and even influence on the communication among consumers, which is necessary to optimally reap the benefits of it. Several diverse examples of OCCNs are illustrated at the cover of this book and listed in Table 1.1.

Communication in OCCNs is prominently characterized by the fact that it provides two distinct categories of value and thus motivation for participants: informational value, which is based on the exchange of productrelated knowledge, and social value, which is based on the enjoyment of interaction (e.g., Dholakia et al. 2004). Furthermore, a prominent characteristic of OCCNs, which has been largely neglected by the literature, is the fact that there are also costs involved for participants, for they have to spend 


\begin{tabular}{|l|l|}
\hline OCCN & URL (d.d. 09-30-2008) \\
\hline c++ & http://www.gidforums.com/f-28.html \\
countries travel & http://www.virtual-countries-travel.com \\
ellegirl & http://forum.ellegirl.nl \\
ford & http://www.ford-world.nl \\
health & http://www.healthboards.com \\
hp & http://forums.itrc.hp.com \\
hyves & http://www.hyves.nl \\
parenting & http://www.iparenting.com \\
jong katholiek & http://www.jongkatholiek.nl \\
pussokram & http://www.csc.kth.se/ pholme/ \\
& presentations/slides/doksem.pdf \\
\hline
\end{tabular}

Table 1.1: Examples of OCCNs

time and effort to maintain their communication links with other members: in the first half of 2008, an average consumer spent $37 \%$ of her online time on communication, the other categories being commerce, content, and search (Internet Activity Index ${ }^{1}$ ).

\subsection{An Economic Exploration of their Formation and Value}

Since its short existence, the marketing literature on this topic has mainly focused on the question why individuals choose to participate in and contribute to online communities (e.g., Bagozzi and Dholakia 2006, Wiertz 2005) and until now disregarded the particulars of these so-called "webs of personal relationships in cyberspace" (Rheingold 2000, p.2)). Yet, structures of who communicates with whom can be empirically observed (e.g., Holme et al. 2004, Fisher et al. 2006) and affect outcomes such as the extent to which value is shared throughout the network and how it is distributed (e.g., Granovetter 2005, Ren et al. 2007). These are important variables since they

\footnotetext{
${ }^{1}$ http://www.online-publishers.org/page.php/prmID/421, retrieved on September, 11th 2008
} 
determine how beneficial OCCNs are for consumers as well as firms.

The economics discipline studies human choices on scarce commodities as means to achieve ends (Robbins 1932). As mentioned before, consumerto-consumer communication is costly (scarcity) and its structure is an important determinant of value (end), so consumers' choices in the formation of a communication network perfectly fit in the field of economics. Since marketing is a multidisciplinary science, grounding its consumer behavior theories on sociology, anthropology, philosophy, psychology, and economics, it is striking that the economic perspective on OCCN formation is clearly lacking in the existing marketing literature.

The current dissertation aims at filling this gap by initiating an economic exploration of the formation and value of OCCNs. To diversify our learning, we focus on distinct but highly interwoven aspects: (i) the strategic aspect, where participants choose to create links based on a cost-benefit tradeoff when the effects of these choices also depend on others' choices, and (ii) the behavioral aspect, where the complexity of the network setting can influence the mental decision rules that individual participants use for link formation.

\subsection{Methods and Outline}

\subsubsection{The Strategic Aspect}

To model the network formation process in its strategic aspect, we use the economic tool for studying strategic interactions, i.e., game theory. This sub-discipline intends to mathematically capture situations where decisionmakers can freely base their choices on the alternatives' costs and benefits, which also depend on the choices of other decision-makers. Recently, a game-theoretic literature stream is developing that is focusing on network formation, conceptualized as a process in which individuals choose to create and maintain links if the participants in the link benefit from doing so, which results in a stable network structure (e.g., Bala and Goyal 2000, Jackson and 
Wolinsky 1996). Although these models are very appropriate if applied to an online setting, this has not been done before.

For this application, we incorporate the mentioned benefit- and costrelated characteristics of OCCNs in our game-theoretical model of network formation. We are first to distinguish between social and informational value as motivations for bilateral exchange decisions, ${ }^{2}$ where informational value can be transferred relatively easily to third parties through indirect links, whereas social value is more individual and therefore not transferable. Moreover, we introduce link specificity in the communication context, meaning that the more direct connections an individual has to maintain with other individuals, the less she is able to specify her attention per link within her total time available. Therefore, her additive value per link for others declines and she also derives less additive value from each link with others (Currarini 2007, Jackson and Wolinsky 1996).

Our game-theoretic model is presented in Chapter 2 of this dissertation. For homogeneous participants and a fixed level of link specificity, the model predicts which network structures emerge dependent on the level of focus on informational versus social value in the community. We first characterize the set of stable structures in the case with only social value to indicate the separate impact of link specificity and demonstrate that it includes a wide range of non-standard architectures. Then in the case with both social and informational value, the joint effect of link specificity and value transferability is shown to reduce the stable set to fragmented architectures consisting of small star components. Finally, the best and worst stable structures from a welfare point of view are described and the role of the relative community focus on informational versus social value is illustrated.

In Chapter 3, the model is generalized as to allow for heterogeneous participants and a variable level of link specificity. By simulations, we show which networks emerge for three levels of link specificity, three levels of focus

\footnotetext{
${ }^{2}$ Note that the marketing literature as mentioned before only considered these value categories as motivations to actively participate in OCCNs in general.
} 
on informational versus social value from communication, and four valuation patterns: homogeneity and expertise heterogeneity, each with and without the presence of one especially highly valued expert. It is illustrated that the structure properties of these networks heavily depend on these parameter values, which results in efficiency and equity differences among settings. Accordingly, we assess the effectivity of several managerial tools for OCCNs. For example, we find that the effects of an expert in a community depend on whether the rest of the community is homogeneous or not. Also, when the informational and social aspects of communication are equally valued, more focus on informational value turns out to be beneficial for community welfare. Finally, enhancing communication in the community by decreasing link specificity does not always lead to higher welfare from communication.

\subsubsection{The Behavioral Aspect}

To investigate the individual link choice process in its behavioral aspect, we use an experimental economics approach. This branch studies choices by having people make them in a controlled laboratory environment, where the consequences of these choices are paid out to participants in monetary terms. There exists a very recent and increasing literature in which researchers experimentally investigate the network formation process (e.g., Callander and Plott 2005, Deck and Johnson 2004). These studies are all involved with the strategic aspect of network formation, whereas we exclude this aspect in order to be able to better examine the behavioral aspect, since the effects of both aspects can be entangled.

For our inquiry how the complexity of the network setting can influence the mental decision rules that participants use for link formation, we combine the experimental economics approach with an individual choice modeling approach as known from the discipline of choice analysis (McFadden 2001, Hensher et al. 2005). It allows us to investigate the impact of important network properties on decisions without having to make strong assumptions about the strategic interplay among heterogeneous, noisy decision-makers. 
Chapter 4 describes our experiment and its results, demonstrating that individuals systematically simplify more complex components of network payoff in their linking decisions. Specifically, they focus on only part of the normative payoff, namely on their own, direct payoff, and tend to ignore indirect payoff and payoff for others in the network. Additionally, individuals use descriptive behavioral traits of link choice alternatives to guide their choices: they are sensitive to whether an alternative involves link deletion or creation and whether it concerns an isolated or a central node. Furthermore, we find that complexity of one type can moderate individuals' dealing with a complex feature of another type. These behavioral effects have important implications for researchers and managers working in areas that involve network formation.

\subsubsection{Outline}

In Figure 1.1, the topic, perspective, and methods of this dissertation are summarized. 


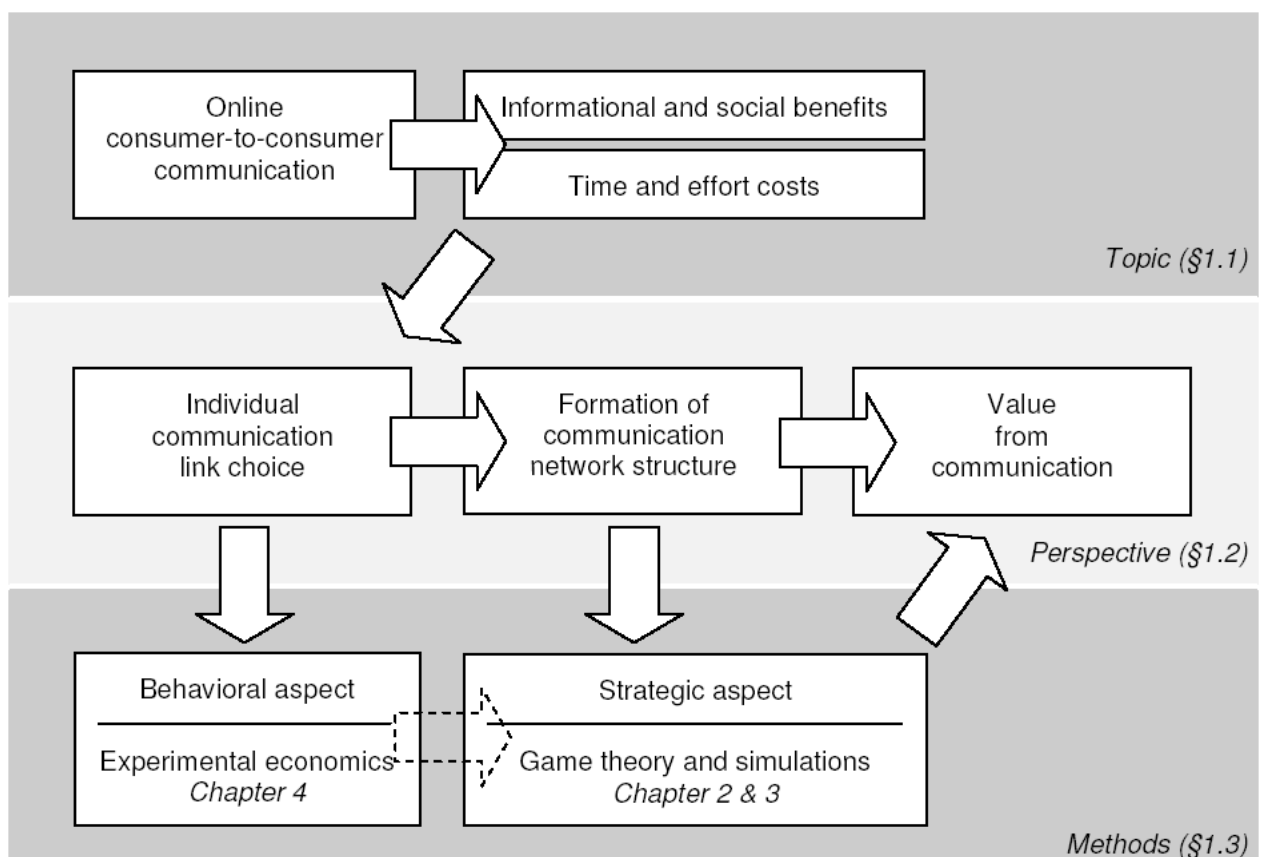

Figure 1.1: Dissertation Summary 


\section{Chapter 2}

\section{The Strategic Aspect: A Game-Theoretical Model}

We analyze the structure of bilateral communication links among consumers in virtual communities by a game-theoretic model of network formation. First, link specificity is incorporated, meaning that the more direct links somebody has to maintain with others, the less she is able to specify her attention per link within her time available, so that the value of her links decreases. Second, a distinction is made between the social and informational value from communication, where informational value is transferable via indirect links, whereas social value is not. We characterize the set of pairwise stable structures in the case with only social value to indicate the separate impact of link specificity and demonstrate that it includes a wide range of non-standard architectures. In the case with both social and informational value, the joint effect of link specificity and value transferability is shown to reduce the pairwise stable set to fragmented architectures consisting of small star components. Finally, we describe the best and worst stable structures from a welfare point of view and illustrate the role of the relative community focus on informational versus social value. 


\section{$2.1 \quad$ Introduction}

Websites such as www.healthboards.com, www.ediets.com/community/, and www.saabnet.com allow a growing number of consumers to easily communicate with like-minded individuals based on shared interests around for example products, consumption activities, or personal conditions. Hence, these communication forums are also increasingly valuable for suppliers, since they are media for word-of-mouth and consumer co-production (e.g., Algesheimer et al. 2005, Dellarocas 2003, Hagel and Armstrong 1997).

Compared to the offline world, consumers in these online communities are relatively flexible to choose their communication partners, since by operating online they are less constrained by geographical distance and by existing social networks, like family structures (Wellman et al. 1996, Van Alstyne and Brynjolfsson 2005). The virtual community literature until now has mainly focused on the question why individuals choose to participate in and contribute to online communities (e.g., Bagozzi and Dholakia 2006, McLure Wasko and Faraj 2005) and disregarded the particulars of these so-called "webs of personal relationships in cyberspace" (Rheingold 2000, p.2)). Yet, structures of who communicates with whom are distinguishing empirical phenomena (e.g., Holme et al. 2004, Fisher et al. 2006) and can determine important outcome variables such as the extent to which value is shared throughout the network and how it is distributed (e.g., Granovetter 2005, Ren et al. 2007).

In the current chapter we do study the structure of the bilateral communication links within online consumer communities, to which we therefore refer as Online Consumer Communication Networks (OCCNs). We model their formation as a game-theoretic network formation process in which individuals choose to create and maintain links, only if the participants in the link benefit from doing so, which results in a pairwise stable network structure (Jackson and Wolinsky 1996). Thus, this chapter illustrates how to use the rich game-theoretic literature on network formation (e.g., Bala and 
Goyal 2000, Jackson and Wolinsky 1996) can be used in an applied setting. Recently studied other settings are firm collaboration (e.g., Goyal and Joshi 2003, Belleflamme and Bloch 2004) and crime networks (Calvó-Armengol and Zenou 2004). We demonstrate that our online consumer communication setting is an appealing application area.

We introduce the important distinction between social and informational value as motivations for bilateral exchange decisions. This typology was suggested by the virtual community literature regarding the question why individuals choose to participate in and contribute to such a community as a whole (e.g., Dholakia et al. 2004). Social value is related to the fact that individuals may simply enjoy communicating with others, for example because they find it entertaining or because they feel it enhances their self-worth (e.g., Hennig-Thurau et al. 2004). Informational value refers to the fact that consumers may obtain new valuable knowledge from other consumers when they communicate online. Typically, informational value can be transferred relatively easily to third parties through indirect links, whereas social value is even more personal and therefore hardly transferable (without creating a direct link). This transferability is more prominent in online than in offline communication since information can be more easily forwarded to others (Wellman et al. 1996).

To analyze the underlying structure of OCCNs we develop a model for the formation of links that allows us to understand the relative impact of social and informational member orientation on the emerging network structures. We incorporate a combination of two important aspects common to OCCNs that has not been investigated before. First, our model features link specificity in the sense that the more direct connections an individual has to maintain with other individuals, the less she is able to specify her attention per link within her total time available. Therefore, her additive value per link for others declines and she also derives less additive value from each link with others (Currarini 2007, Jackson and Wolinsky 1996). Second, we realize that when the value derived from communication is not only social but also contains an informational component, this is transferable via indirect 
links (Bala and Goyal 2000).

More specifically, we first deal with the case of communication having social value only (Section 2.2) in order to illustrate the separate impact of link specificity on network structure. The set of pairwise stable structures is characterized by two simple conditions and is shown to contain a wide range of non-standard architectures, including highly connected and "small world" structures, whereas previous models for social and economic network formation mostly predicted simple architectures like stars and wheels.

Next, we deal with the case of communication from which both social and informational value is derived (Section 2.3) in order to illustrate the impact of value transferability on structure. Only structures that consist of disjoint star components of two or three agents are shown to be pairwise stable. Apparently, the combination of these two features: link specificity, which is an example of a negative network externality, and informational value transferability, which is an example of a positive network externality (Asvanund et al. 2004), has a strong fragmentizing effect on the emerging pairwise stable network structures.

Subsequently, Section 2.4 characterizes the best and worst pairwise stable network structures from a welfare point of view. It follows that a mix of social and informational focus is better than a purely social focus. Therefore, the familiar tension between stability and efficiency (e.g., Jackson and Wolinsky 1996) can be diminished by introducing value transferability into a setting with link specificity. We also provide further guidelines for when it is better to stimulate either the social or the informational orientation of an OCCN. Section 2.5 concludes and offers directions for further research.

\subsection{Social Value}

Although we believe that OCCNs typically combine social and informational value aspects in their communication, we first deal with the simpler case in 
which only social value is derived from communication. This approach allows us to illustrate the separate impact of link specificity on structure and welfare. Link specificity (Currarini 2007, Jackson and Wolinsky 1996) implies that the more direct connections an individual has to maintain with other individuals, the less she is able to specify her attention per link within her total time available. Therefore, her additive value per link for others declines and she also derives less additive value from each link with others. These negative externalities of link formation are crucial in our communication context, since here no benefits arise from individual contributions as such. The reason is that communication is only valuable if it is twosided, thus time and effort have to be invested by both sender and receiver. ${ }^{1}$ The objective of this section therefore is to develop a model for network formation in OCCNs with only social value from communication. We also propose an appropriate stability concept and the large category of stable network structures is characterized. ${ }^{2}$

\subsubsection{Model}

Consider a community of agents $N=\{1, \ldots, n\}, n \geq 3$. A direct link $g_{i, j}$ between agents $i$ and $j$ in this community $(i, j \in N ; i \neq j)$ can be interpreted as a virtual communication relationship between $i$ and $j$ which is established if they both wish the link. These relationships are expressed by undirected links: for any two agents $i$ and $j, g_{i, j}=g_{j, i}$. By definition, $g_{i, i}=0$, as agents do not establish communication links with themselves. In this community agents only derive social value from interaction.

In case of an isolated relationship between two agents, each agent experiences social value $V^{\mathrm{s}}>0$ as the outcome of their joint communication

\footnotetext{
${ }^{1}$ In contrast, in the co-author setting, which has been the subject of investigation in earlier research (Jackson and Wolinsky 1996), each co-author can write independently as well.

${ }^{2}$ We do recognize that next to the value derived from relationships with specific other participants within an OCCN, participants can also derive value from the community as a whole (cf. Ren et al. 2007). However, since this is not expected to influence the specific linking decisions they make, we assume it to be constant in our model.
} 
production process. However, maintenance of the communication relationship costs effort: investment of both agents is needed in order to make the communication specific to their personal circumstances and hence useful. Accordingly, in case of a structure where two agents do not form an isolated pair, both agents are assumed to divide their effort equally among all their relationships, as a result of which the potential social communication value is divided proportionally.

Next, we assume that the contributions of two agents in their bilateral process of communication value creation are equal and complementary. Therefore, the total payoff for agent $i$ in link structure $g$ is given by

$$
\Pi_{i}(g)=\begin{array}{cc}
\sum_{j \in N_{i}(g) \frac{V^{\mathrm{s}}}{\mu_{i}(g) \cdot \mu_{j}(g)}} & \text { if } \mu_{i}(g)>0 \\
0 & \text { if } \mu_{i}(g)=0,
\end{array}
$$

where an element of matrix $g$ written as $g_{i, j}$ indicates with a 1 or a 0 whether $i$ is directly linked to $j$ or not; $N_{i}(g)$ is the set of agents with whom $i$ has a direct link, where agent $j$ is a neighbor of agent $i$ if $j \in N_{i}(g)$, and $\mu_{i}(g)=\left|N_{i}(g)\right|$ is the number of neighbors of agent $i$, which can also be referred to as the degree of $i$; and $V^{\mathrm{s}}>0$ denotes the social value that $i$ would derive from communication with $j$ if neither $i$ nor $j$ were linked to any other agent. Observe that agents' contributions are reflected by a Cobb-Douglas production function with unit output elasticities. ${ }^{3}$

For the model thus described stable network structures are predicted to emerge by using the concept of pairwise stability (Jackson and Wolinsky 1996), where a network structure is stable if no single agent can strictly improve her payoff by deleting one of her direct links and no pair of agents can strictly improve her sum of payoffs while each of the two members is at least indifferent by creating a direct link between the two members. Therefore, this solution concept is weak in the sense that it only assumes stability against deviations of exactly one link (which involves the permission

\footnotetext{
${ }^{3}$ For comparison: the payoff function in the co-author model of Jackson and Wolinsky (1996) can be written as $\Pi_{i}(g)=\sum_{j \in N_{i}(g)}\left(\frac{V^{\mathrm{s}}}{\mu_{i}(g)}+\frac{V^{\mathrm{s}}}{\mu_{j}(g)}+\frac{V^{\mathrm{s}}}{\mu_{i}(g) \cdot \mu_{j}(g)}\right)$.
} 
of two agents in the case of link formation), reflecting a form of myopia. Alternatively, the model could be analyzed by applying the solution concept of Nash equilibrium (Bala and Goyal 2000), which assumes stability against single-agent deviations of more than one link. If the strategies $g_{i, j}$ indicate with a 0 or a 1 whether $i$ wants to be directly linked to $j$ or not and again the actual links $\bar{g}_{i, j}=\min \left\{g_{i, j}, g_{j, i}\right\}$ are only formed if both agents want the link, the payoff function could be written as

$$
\Pi_{i}(g)=\sum_{j \in N_{i}(\bar{g})} \frac{V^{\mathrm{s}}}{\mu_{i}(\bar{g}) \cdot \mu_{j}(\bar{g})} .
$$

Now $\bar{g}$ would be defined as a Nash network if it is supported by some $g$ from which no single agent can change her strategies such that she strictly improves her payoff. If $n=3$, all possible structures would be Nash networks. For example, the empty network would be Nash since it is supported by the strategies $g_{i, j}=0$ for all $i, j \in N$, even though agents would obviously benefit from changing their strategies simultaneously. Because of this extreme coordination problem of the Nash concept in two-sided link formation and since the weak concept of pairwise stability already clearly and interestingly constrains the number of network structures that are stable, we choose for the pairwise stability solution.

In our notation, we have the following definition.

Definition 1 (pairwise stability) The structure $g$ is pairwise stable if for all $i, j \in N$ with $g_{i, j}=1$ it holds that

$$
\Pi_{i}(g) \geq \Pi_{i}\left(g^{\prime}\right) \text { and } \Pi_{j}(g) \geq \Pi_{j}\left(g^{\prime}\right)
$$

where $g^{\prime}$ is such that $g_{i, j}^{\prime}=0$ and $g_{k, l}^{\prime}=g_{k, l}$ for all $\{k, l\} \neq\{i, j\}$, and for all $i, j \in N$ with $g_{i, j}=0$ it holds that

$$
\begin{gathered}
\Pi_{i}(g)>\Pi_{i}\left(g^{\prime}\right) \text { or } \\
\Pi_{j}(g)>\Pi_{j}\left(g^{\prime}\right) \text { or }
\end{gathered}
$$




$$
\left(\Pi_{i}(g)=\Pi_{i}\left(g^{\prime}\right) \text { and } \Pi_{j}(g)=\Pi_{j}\left(g^{\prime}\right)\right),
$$

where $g^{\prime}$ is such that $g_{i, j}^{\prime}=1$ and $g_{k, l}^{\prime}=g_{k, l}$ for all $\{k, l\} \neq\{i, j\}$.

\subsubsection{Results}

We prove that the class of pairwise stable network structures can be described by two easily verifiable conditions: (i) they are what we call equal neighbor degree structures, meaning that everybody has at least one neighbor and every neighbor of agent $i$ has the same degree, and (ii) there is at most a difference of one between the degrees of agents in the same component.

Definition 2 (equal neighbor degree structure) A structure $g$ is an equal neighbor degree structure when it holds for each agent $i$ that $\mu_{i}(g) \geq 1$ and for all agents $j, j^{\prime} \in N_{i}(g)$ that $\mu_{j}(g)=\mu_{j^{\prime}}(g)$.

Definition 3 (component) A component in a network $g$ is a maximal set of agents $C \subseteq N$ who are connected to one another directly or indirectly, so for each $i, j \in C$ there exists a sequence of agents $k_{1}, \ldots, k_{m} \in C$ for whom it holds that $g_{i, k_{1}}=g_{k_{1}, k_{2}}=\ldots=g_{k_{m-1}, k_{m}}=g_{k_{m}, j}=1$.

First consider the following lemma, in which the own degree of agent $i$ is denoted by $d_{i}$ and her neighbors' degree by $e_{i}$.

Lemma 4 A structure is pairwise stable if and only if it is an equal neighbor degree structure where it holds for each not directly linked pair of agents $i, j$ that

$$
e_{i} \leq d_{j} \text { or } e_{j} \leq d_{i} \text { or }\left(e_{i}=d_{j}+1 \text { and } e_{j}=d_{i}+1\right)
$$

Proof. $(\Longleftarrow)$ Assume that $g$ is an equal neighbor degree structure where for each not directly linked pair of agents $i, j$ condition (2.2) is satisfied. The 
payoff of an agent $k$ as expressed in equation (2.1) reduces to $d_{k} V^{\mathrm{s}} /\left(d_{k} e_{k}\right)$, so agent $k$ does not want to delete a link, for

$$
d_{k} \frac{V^{\mathrm{s}}}{d_{k} e_{k}} \geq\left(d_{k}-1\right) \frac{V^{\mathrm{s}}}{\left(d_{k}-1\right) e_{k}} .
$$

Moreover, no link between any pair of agents $i, j$ is created if it makes either $i$ or $j$ strictly worse off or both of them equally well off. Therefore, no link is created if

$$
\begin{gathered}
\frac{V^{\mathrm{s}}}{e_{i}}>d_{i} \frac{V^{\mathrm{s}}}{\left(d_{i}+1\right) e_{i}}+\frac{V^{\mathrm{s}}}{\left(d_{i}+1\right)\left(d_{j}+1\right)} \quad \text { or } \\
\frac{V^{\mathrm{s}}}{e_{j}}>d_{j} \frac{V^{\mathrm{s}}}{\left(d_{j}+1\right) e_{j}}+\frac{V^{\mathrm{s}}}{\left(d_{i}+1\right)\left(d_{j}+1\right)} \quad \text { or } \\
\left(\begin{array}{c}
\frac{V^{\mathrm{s}}}{e_{i}}=d_{i} \frac{V^{\mathrm{s}}}{\left(d_{i}+1\right) e_{i}}+\frac{V^{\mathrm{s}}}{\left(d_{i}+1\right)\left(d_{j}+1\right)} \\
\text { and } \frac{V^{\mathrm{s}}}{e_{j}}=d_{j} \frac{V^{\mathrm{s}}}{\left(d_{j}+1\right) e_{j}}+\frac{V^{\mathrm{s}}}{\left(d_{i}+1\right)\left(d_{j}+1\right)}
\end{array}\right) .
\end{gathered}
$$

The following illustrates that $e_{i} \leq d_{j}$ implies (2.3):

$e_{i} \leq d_{j} \Longrightarrow d_{i}\left(d_{j}+1\right)+e_{i}<\left(d_{i}+1\right)\left(d_{j}+1\right) \Longrightarrow \frac{d_{i}\left(d_{j}+1\right)+e_{i}}{\left(d_{i}+1\right)\left(d_{j}+1\right) e_{i}}<\frac{1}{e_{i}}$.

Analogously, it can be shown that $e_{j} \leq d_{i}$ implies (2.4), and $\left(e_{i}=d_{j}+1\right)$ and $\left(e_{j}=d_{i}+1\right)$ implies (2.5). Therefore, $g$ is pairwise stable.

( $\Longrightarrow$ ) Assume that the structure $g$ is pairwise stable. First, suppose that there is an agent $i$ for whom it holds that $\mu_{i}(g)=0$. Then her payoff would strictly improve from a link with some other agent $k$. It is obvious that also $k$ 's payoff would strictly increase if $\mu_{k}(g)=0$, which contradicts pairwise stability, so consider the case where $\mu_{k}(g) \geq 1$. The payoff of $k$ without this link equals

$$
\sum_{j \in N_{k}(g)} \frac{V^{\mathrm{s}}}{\mu_{k}(g) \cdot \mu_{j}(g)}=\frac{V^{\mathrm{s}}}{\mu_{k}(g)}\left(\sum_{j \in N_{k}(g)} \frac{1}{\mu_{j}(g)}\right)
$$


whereas by linking with $i$ it would become

$$
\begin{aligned}
& \sum_{j \in N_{k}(g)} \frac{V^{\mathrm{s}}}{\left(\mu_{k}(g)+1\right) \cdot \mu_{j}(g)}+\frac{V^{\mathrm{s}}}{\left(\mu_{k}(g)+1\right) \cdot 1} \\
= & \frac{V^{\mathrm{s}}}{\left(\mu_{k}(g)+1\right)}\left(\sum_{j \in N_{k}(g)} \frac{1}{\mu_{j}(g)}+1\right) \\
\geq & \frac{V^{\mathrm{s}}}{\mu_{k}(g)}\left(\sum_{j \in N_{k}(g)} \frac{1}{\mu_{j}(g)}\right) .
\end{aligned}
$$

The inequality follows from the observation that the expression before the inequality equals $V^{\mathrm{s}}$ times the average of the terms $1 / \mu_{j}(g)$ and 1 , the expression after the inequality is equal to $V^{\mathrm{s}}$ times the average of the terms $1 / \mu_{j}(g)$, and that $1 \geq 1 / \mu_{j}(g)$ for all $j \in N_{j}(g)$. This also contradicts pairwise stability. It follows that $\mu_{i}(g) \geq 1$ for all $i \in N$.

Secondly, suppose that for some $i$ it does not hold that $\mu_{j}(g)$ is constant for all $j \in N_{i}(g)$. Then there is an agent $k \in N_{i}(g)$ such that

$$
\mu_{k}(g)>\frac{\sum_{j \in N_{i}(g)} \mu_{j}(g)}{\mu_{i}(g)} .
$$

The payoff for $i$ is given by

$$
\sum_{j \in N_{i}(g)} \frac{V^{\mathrm{s}}}{\mu_{i}(g) \cdot \mu_{j}(g)}=\frac{V^{\mathrm{s}}}{\mu_{i}(g)} \sum_{j \in N_{i}(g)} \frac{1}{\mu_{j}(g)},
$$

whereas by deleting the link with $k$, the payoff for $i$ would become

$$
\begin{aligned}
& \sum_{j \in N_{i}(g)} \frac{V^{\mathrm{s}}}{\left(\mu_{i}(g)-1\right) \cdot \mu_{j}(g)}-\frac{V^{\mathrm{s}}}{\left(\mu_{i}(g)-1\right) \cdot \mu_{k}(g)} \\
= & \frac{V^{\mathrm{s}}}{\left(\mu_{i}(g)-1\right)}\left(\sum_{j \in N_{i}(g)} \frac{1}{\mu_{j}(g)}-\frac{1}{\mu_{k}(g)}\right) \\
> & \frac{V^{\mathrm{s}}}{\mu_{i}(g)} \sum_{j \in N_{i}(g)} \frac{1}{\mu_{j}(g)},
\end{aligned}
$$


where the last inequality follows immediately from the interpretation of the last two terms as $V^{\mathrm{s}}$ times an average of numbers $1 / \mu_{j}(g)$. This contradicts pairwise stability, so $\mu_{j}(g)=\mu_{j^{\prime}}(g)$ for all $j, j^{\prime} \in N_{i}(g)$. We have shown that a pairwise stable structure is an equal neighbor degree structure.

Finally, suppose that there exists a not directly linked pair $i, j$ for which condition (2.2) is not satisfied, implying

$$
e_{i} \geq d_{j}+1 \text { and } e_{j} \geq d_{i}+1 \text { and }\left(e_{i}>d_{j}+1 \text { or } e_{j}>d_{i}+1\right) \text {. }
$$

Then $i$ and $j$ want to create a link between them, since this would cause the payoff for agent $i$ to become

$$
d_{i} \frac{V^{\mathrm{s}}}{\left(d_{i}+1\right) e_{i}}+\frac{V^{\mathrm{s}}}{\left(d_{i}+1\right)\left(d_{j}+1\right)} \geq d_{i} \frac{V^{\mathrm{s}}}{\left(d_{i}+1\right) e_{i}}+\frac{V^{\mathrm{s}}}{\left(d_{i}+1\right) e_{i}}=\frac{V^{\mathrm{s}}}{e_{i}},
$$

and for agent $j$ to become

$$
d_{j} \frac{V^{\mathrm{s}}}{\left(d_{j}+1\right) e_{j}}+\frac{V^{\mathrm{s}}}{\left(d_{j}+1\right)\left(d_{i}+1\right)} \geq d_{j} \frac{V^{\mathrm{s}}}{\left(d_{j}+1\right) e_{j}}+\frac{V^{\mathrm{s}}}{\left(d_{j}+1\right) e_{j}}=\frac{V^{\mathrm{s}}}{e_{j}}
$$

where according to the last condition in (2.6) at least one of the $\geq$ - signs is strict. This contradicts pairwise stability as well. Therefore, $g$ is an equal neighbor degree structure with

$$
e_{i} \leq d_{j} \text { or } e_{j} \leq d_{i} \text { or }\left(e_{i}=d_{j}+1 \text { and } e_{j}=d_{i}+1\right)
$$

for each not directly linked pair of agents $i, j$.

Condition (2.2) in Lemma 4 can be further simplified as is shown in the following proposition.

Proposition 5 A structure is pairwise stable if and only if it is an equal neighbor degree structure where it holds for each pair of agents $k, l$ in the same component that

$$
\left|d_{k}-d_{l}\right| \leq 1
$$


Proof. Considering Lemma 4, it is sufficient to show that in an equal neighbor degree structure condition (2.2) holds for each not directly linked pair $i, j$ if and only if condition (2.7) is satisfied for each pair $k, l$ in the same component.

$(\Longleftarrow)$ Assume an equal neighbor degree structure where for each pair $k, l$ in the same component condition (2.7) is satisfied. Let $i, j$ be any not directly linked pair. If $e_{i} \leq d_{j}$, condition (2.2) is satisfied. If not, then $e_{i}>d_{j}$ and we can derive by applying condition (2.7) twice that

$$
e_{j} \leq d_{j}+1 \leq e_{i} \leq d_{i}+1
$$

If $e_{j} \leq d_{i}$, condition (2.2) is satisfied. If not, then $e_{j}=d_{i}+1$ and condition (2.2) is satisfied if it also holds that $e_{i}=d_{j}+1$. Suppose not, then $e_{i} \geq d_{j}+2$ and we can derive by applying condition (2.7) that

$$
e_{i} \geq d_{j}+2 \geq\left(e_{j}-1\right)+2=d_{i}+2,
$$

which contradicts condition (2.7). Therefore, condition (2.2) is satisfied.

( $\Longrightarrow$ ) Assume an equal neighbor degree structure where for each not directly linked pair $i, j$ condition (2.2) is satisfied. Let $k, l$ be any pair in the same component. There exists at least one path between $k$ and $l$. Assume that the number of other agents on any of these paths is odd. Due to the equal neighbor degree structure it holds that

$$
e_{k}=e_{l} \text { and } d_{k}=d_{l},
$$

so condition (2.7) is satisfied. Assume that the number of other agents on all of these paths is even. If the component consists of only $k$ and $l$, then condition (2.7) trivially holds. Otherwise, there either exists an agent $m \in N_{l}(g), m \neq k$, or there exists an agent $n \in N_{k}(g), n \neq l$. Without loss of generality, assume the former is the case. Since all paths between $k$ and $l$ have an even number of other agents, it follows that $m$ is not directly 
linked to $k$. Due to the equal neighbor degree structure it holds that

$$
e_{k}=e_{m}, d_{m}=d_{k}, e_{m}=d_{l}, \text { and } e_{l}=d_{m}
$$

Suppose that condition (2.7) is not satisfied, so without loss of generality:

$$
d_{l} \geq d_{k}+2
$$

Then it follows that

$$
e_{k} \geq d_{m}+2 \text { and } e_{m} \geq d_{k}+2
$$

which contradicts condition (2.2) for $k, m$. Consequently, condition (2.7) is satisfied.

The following examples illustrate the wide range of structures thus proven to be pairwise stable in the social value case.

Definition 6 (complete structure) A structure $g$ is complete if all agents are connected, so for all $i, j \in N$ it holds that $g_{i, j}=1$.

Definition 7 (wheel structure) A structure $g$ is a wheel if it has exactly $n$ links and there exists a sequence of different agents $k_{1}, \ldots, k_{n} \in N$ for whom it holds that $g_{k_{1}, k_{2}}=g_{k_{2}, k_{3}}=\ldots=g_{k_{n-1}, k_{n}}=g_{k_{n}, k_{1}}=1$.

Definition 8 (regular structure) A structure $g$ is regular if it exists of one component and for each agent $i \in N$ it holds that $d_{i}=d$.

Corollary 9 The complete, wheel, or any regular structure is pairwise stable, for it is an equal neighbor degree structure where it holds for each pair of agents $k, l$ in the single component that

$$
\left|d_{k}-d_{l}\right|=0 \leq 1
$$


Example 10 A non-regular structure that is pairwise stable is given in $\mathrm{Fi}$ gure 2.1.

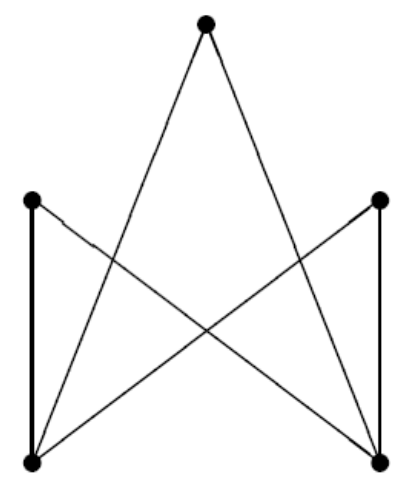

Figure 2.1: A Non-Regular Pairwise Stable Structure (Without Informational Value)

Example 11 A structure consisting of multiple components that is pairwise stable is given in Figure 2.2.

Example 12 A "small world" is a structure with local clusters of highly interlinked agents together with agents that link the various clusters. As a consequence, although most agents are not directly connected, every agent is indirectly linked to every other agent by a relatively small number of steps. A "small world" structure that is pairwise stable is given in Figure 2.3.

Note that this wide set of stable structures includes complex real-life architectures (e.g., Dodds et al. 2003), whereas previous models for social and economic network formation mostly predicted simple architectures like stars and wheels (e.g., Bala and Goyal 2000, Goyal and Vega-Redondo 2007). ${ }^{4}$

\footnotetext{
${ }^{4}$ Our results also differ with respect to the co-author model (Jackson and Wolinsky 1996) as this predicts that a pairwise stable structure can be partitioned into fully intraconnected components, each of which has a different number of members. If $m_{1}$ is the number of members of one such component and $m_{2}$ is the next largest in size, then $m_{1}>\left(m_{2}\right)^{2}$.
} 


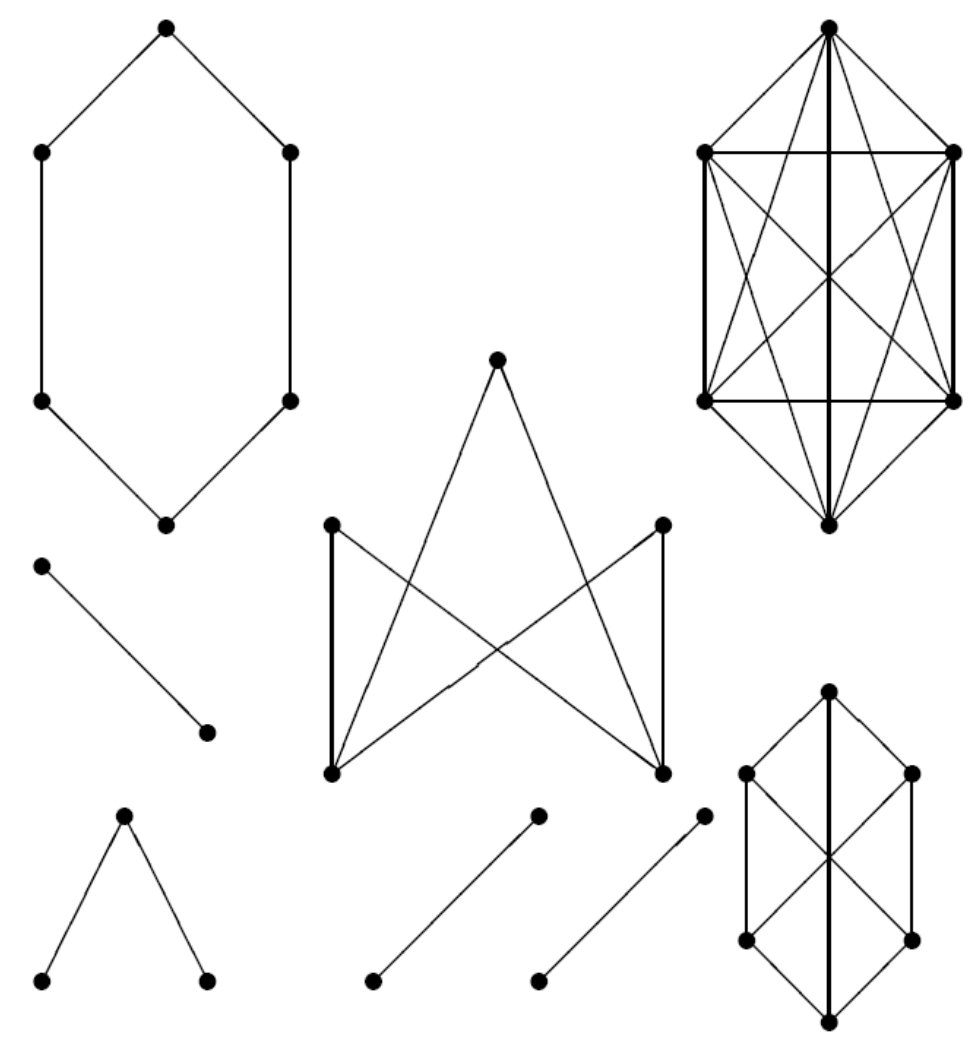

Figure 2.2: A Multiple-Component Pairwise Stable Structure (Without Informational Value)

\subsection{Informational as well as Social Value}

This section introduces the case in which both social and informational value is derived from communication in OCCNs. Thus, we can illustrate the impact of value transferability on structure and welfare along with the effect of link specificity. Value transferability (Bala and Goyal 2000) means that value from communication is not only derived by direct neighbors, but can also be transferred via indirect links. More specifically, we make a distinction between social and informational value derived from communication, where 


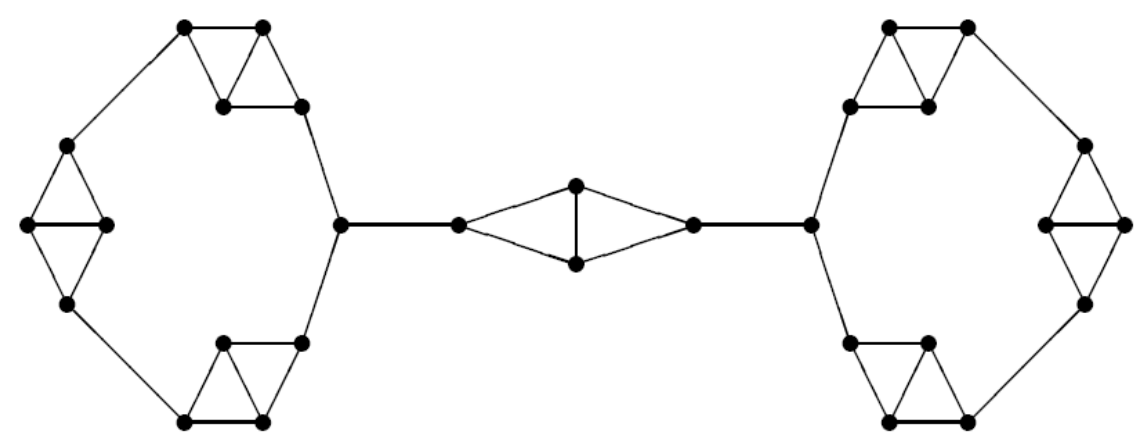

Figure 2.3: A "Small World" Pairwise Stable Structure (Without Informational Value)

only informational value is transferable through the network. For example, social value from communication between two Saab enthusiasts only exists for the two communication partners, but informational value (e.g., from a solution to a technical problem) can (partly) exist for others in the network. After proposing a model for network formation in this setting, the pairwise stable network structures are characterized again. We show that the set of stable structures is much more limited in range than in the social value setting.

\subsubsection{Model}

Consider a community of agents $N=\{1, \ldots, n\}, n \geq 3$. A direct link $g_{i, j}$ between agents $i$ and $j$ in this community $(i, j \in N ; i \neq j)$ can be interpreted as a virtual communication relationship between $i$ and $j$ which is established if they both wish the link. These relationships are expressed by undirected links: for any two agents $i$ and $j, g_{i, j}=g_{j, i}$, and $g_{i, i}=0$.

In case of an isolated relationship between two agents where interaction only has social value, each agent experiences social value $V^{\mathrm{s}}>0$ as the 
outcome of their joint communication production process. In case of an isolated relationship between two agents where interaction only has informational value, each agent experiences informational value $V^{\mathrm{i}}>0$ as the outcome of their joint communication production process. In general, agents are assumed to give relative attention to informational and social value in the proportions $\alpha$ and $1-\alpha$ respectively, where $\alpha$ is assumed to be constant satisfying $0<\alpha<1 .^{5}$

Maintenance of the communication relationship between two agents requires bilateral effort investment in order to make the communication specific and hence useful. Accordingly, in case of a structure where two agents do not form an isolated pair, both agents are assumed to divide their effort equally among all their relationships, as a result of which the potential informational as well as social communication value is divided proportionally. We assume that the contributions of two agents in their communication value creation are equal and complementary.

Moreover, informational value is, without any decay except for this effort division, transferred to third parties through indirect links (paths of links), whereas social value is not transferable. This is due to the fact that in the direct communication production process of two agents, any of them can use the informational value that she acquired during the bilateral communication creation with other neighbors. Consequently, agent $j_{0}$ experiences not only first-step informational payoff from her direct neighbors:

$$
\Pi_{j_{0}}^{1 \mathrm{i}}(g)=\sum_{j_{1} \in N_{j_{0}}(g)} \frac{V^{\mathrm{i}}}{\mu_{j_{0}}(g) \cdot \mu_{j_{1}}(g)},
$$

which is similar to the social payoff in equation (2.1), but also second-step

\footnotetext{
${ }^{5}$ The results in the case where the value derived from communication is only informational $(\alpha=1)$ slightly differ from those in this mixed case $(0<\alpha<1)$. Specifically, it appears that structures also containing one four-agent star component can be pairwise stable.
} 
informational payoff:

$$
\begin{aligned}
\Pi_{j_{0}}^{2 \mathrm{i}}(g)= & \sum_{j_{1} \in N_{j_{0}}(g)} \frac{1}{\mu_{j_{0}}(g) \cdot \mu_{j_{1}}(g)} \\
& \sum_{j_{2} \in N_{j_{1}}(g) \backslash j_{0}} \frac{V^{\mathrm{i}}}{\mu_{j_{1}}(g) \cdot \mu_{j_{2}}(g)},
\end{aligned}
$$

third-step informational payoff:

$$
\begin{aligned}
\Pi_{j_{0}}^{3 \mathrm{i}}(g)= & \sum_{j_{1} \in N_{j_{0}}(g)} \frac{1}{\mu_{j_{0}}(g) \cdot \mu_{j_{1}}(g)} \sum_{j_{2} \in N_{j_{1}}(g) \backslash j_{0}} \frac{1}{\mu_{j_{1}}(g) \cdot \mu_{j_{2}}(g)} \\
& \sum_{j_{3} \in N_{j_{2}}(g) \backslash j_{1}, j_{0}} \frac{V^{\mathrm{i}}}{\mu_{j_{2}}(g) \cdot \mu_{j_{3}}(g)},
\end{aligned}
$$

and so forth. Thus, the overall informational payoff received by agent $j_{0}$ is equal to

$$
\begin{aligned}
\Pi_{j_{0}}^{\mathrm{i}}(g) & =\sum_{q=1}^{n-1} \Pi_{j_{0}}^{q \mathrm{i}}(g) \\
& =V^{\mathrm{i}} \sum_{q=1}^{n-1} \prod_{x=1}^{q} \sum_{j_{x} \in N_{j_{x-1}}(g) \backslash j_{x-2}, j_{x-3}, \ldots, j_{0}} \frac{\frac{1}{\mu_{j_{x-1}}(g) \cdot \mu_{j_{x}}(g)}}{} \sum_{q=1}^{n-1} \sum_{x=1}^{q} \frac{V^{\mathrm{i}}}{j_{x} \in N_{j_{x-1}}(g) \backslash j_{x-2}, j_{x-3}, \ldots, j_{0}} \frac{\mu_{j_{0}}(g) \cdot \prod_{b=1}^{q-1}\left(\mu_{j_{b}}(g)\right)^{2} \cdot \mu_{j_{q}}(g)}{}
\end{aligned}
$$

Therefore, the total payoff for agent $i$ in link structure $g$ is given by

$$
\Pi_{i}(g)=\left\{\begin{array}{cl}
\alpha \sum_{j \in \bar{N}_{i}(g)} \sum_{p \in \mathcal{P}_{i, j}(g)} \frac{V^{\mathrm{i}}}{\mu_{i}(g) \cdot \prod_{k \in \breve{P}}\left(\mu_{k}(g)\right)^{2} \cdot \mu_{j}(g)} & \text { if } \mu_{i}(g)>0 \\
+(1-\alpha) \sum_{j \in N_{i}(g) \frac{V^{\mathrm{s}}}{\mu_{i}(g) \cdot \mu_{j}(g)}} & \text { if } \mu_{i}(g)=0 \\
0 &
\end{array}\right.
$$


where an element of matrix $g$ written as $g_{i, j}$ indicates with a 1 or a 0 whether $i$ is directly linked to $j$ or not; $\alpha$ is the proportion of communication through each link in the community that concerns product-, service- or firm-related information and $1-\alpha$ is the proportion of communication through each link in the community that concerns social interaction; $N_{i}(g)$ is the set of agents with whom $i$ has a direct link, where agent $j$ is a neighbor of agent $i$ if $j \in N_{i}(g)$, and $\mu_{i}(g)=\left|N_{i}(g)\right|$ is the number of neighbors of agent $i$, which can also be referred to as the degree of $i$, and $\bar{N}_{i}(g)$ is the set of agents with whom $i$ has either a direct or an indirect link; $\mathcal{P}_{i, j}(g)$ is the set of paths between $i$ and $j$, where a path is defined as a sequence of consecutive edges without repeated nodes, and $\breve{P}$ is the set of agents on path $p$ between $i$ and $j$ excluding $i$ and $j$ themselves; and $V^{\mathrm{i}}>0$ denotes the informational value that $i$ would derive from communication with $j$ if neither $i$ nor $j$ were linked to any other agent and interaction would only have informational value, and $V^{\mathrm{s}}>0$ denotes the social value that $i$ would derive from communication with $j$ if neither $i$ nor $j$ were linked to any other agent and interaction would only have social value.

For the model thus described stable network structures are again predicted to emerge by using the concept of pairwise stability (Jackson and Wolinsky 1996).

\subsubsection{Results}

It can be proven that the pairwise stable structures consist of small star components.

Definition 13 (star) A structure $g$ is a star if it has exactly $n-1$ links and there exists an agent $j$ for whom it holds that $g_{j, i}=1$ for all $i \neq j$. Similarly, a component $C$ is a star if it has exactly $|C|-1$ links and it contains an agent $j$ for whom it holds that $g_{j, i}=1$ for any other $i \in C$. Agent $j$ is called the center agent whereas the other agents are the periphery agents of the star. 
First consider the following lemma in which it is shown that the star structure becomes unstable when there are more than three agents.

Lemma 14 The star structure is pairwise stable if and only if $n=3$.

Proof. From the star structure, it is not beneficial for any of the periphery agents to delete her link with the center agent as then her payoff will be zero. For the center agent, deleting a link with any of the periphery agents will provide her with the same payoff. To verify this result, it is crucial to observe that the center agent is not involved in any indirect links to other agents in star structures. Periphery agent $i$ does not create a link with another periphery agent $i$ ' if and only if

$$
\begin{aligned}
& \alpha V^{\mathrm{i}}\left(\frac{1}{n-1}+\frac{(n-2)}{(n-1)^{2}}\right)+(1-\alpha) V^{\mathrm{s}} \frac{1}{n-1} \geq \\
& \alpha V^{\mathrm{i}}\left(\frac{1}{2(n-1)}+\frac{1}{8(n-1)}+\frac{1}{4}+\frac{1}{4(n-1)^{2}}+\frac{(n-3)}{2(n-1)^{2}}+\frac{(n-3)}{8(n-1)^{2}}\right) \\
& +(1-\alpha) V^{\mathrm{s}}\left(\frac{1}{2(n-1)}+\frac{1}{4}\right) \\
& \Longleftrightarrow \alpha V^{\mathrm{i}}(4-n)+(1-\alpha) V^{\mathrm{s}}(3-n) \geq 0 \Longleftrightarrow n \leq 3 \text {. }
\end{aligned}
$$

Since we assumed societies to consist of at least three agents, it holds that $n=3$.

Now the following proposition can be proven.

Proposition 15 A structure is pairwise stable if and only if it consists of disjoint star components of two or three agents.

Proof. $(\Longleftarrow)$ It is not beneficial for any of the periphery agents in a star component to delete her single link as then her payoff will be zero. Equivalently, for the center agent in a three-agent component, deleting a link with 
any of the two periphery agents is not beneficial as it will provide her with the same payoff.

Link creation between the periphery agents of one three-agent star is eliminated by Lemma 14. Therefore, we only have to examine the following cases $(a)-(f)$ :

\begin{tabular}{cl|c|c|c} 
& & 2 & $3 \mathrm{c}$ & $3 \mathrm{p}$ \\
\hline 2 & (pair agent) & $(a)$ & $(b)$ & $(c)$ \\
\hline $3 \mathrm{c}$ & (center agent of three-agent star) & $\mathrm{x}$ & $(d)$ & $(e)$ \\
\hline $3 \mathrm{p}$ & (periphery agent of three-agent star) & $\mathrm{x}$ & $\mathrm{x}$ & $(f)$
\end{tabular}

For each of these cases, it can be proven by evaluating the payoffs with and without the link that no link is created: in case $(a)$, a pair agent would get payoff

$$
\alpha V^{\mathrm{i}}\left(\frac{1}{2}+\frac{1}{4}+\frac{1}{8}\right)+(1-\alpha) V^{\mathrm{s}}\left(\frac{1}{2}+\frac{1}{4}\right) \leq \alpha V^{\mathrm{i}}+(1-\alpha) V^{\mathrm{s}},
$$

in case $(b)$, the pair agent would get payoff

$$
\alpha V^{\mathrm{i}}\left(\frac{1}{2}+\frac{1}{6}+\frac{1}{18}+\frac{1}{18}\right)+(1-\alpha) V^{\mathrm{s}}\left(\frac{1}{2}+\frac{1}{6}\right)<\alpha V^{\mathrm{i}}+(1-\alpha) V^{\mathrm{s}}
$$

in case $(c)$, the pair agent would get payoff

$$
\alpha V^{\mathrm{i}}\left(\frac{1}{2}+\frac{1}{4}+\frac{1}{16}+\frac{1}{32}\right)+(1-\alpha) V^{\mathrm{s}}\left(\frac{1}{2}+\frac{1}{4}\right)<\alpha V^{\mathrm{i}}+(1-\alpha) V^{\mathrm{s}}
$$

in case $(d)$, a center agent would get payoff

$$
\begin{aligned}
& \alpha V^{\mathrm{i}}\left(\frac{1}{3}+\frac{1}{3}+\frac{1}{9}+\frac{1}{27}+\frac{1}{27}\right)+(1-\alpha) V^{\mathrm{s}}\left(\frac{1}{3}+\frac{1}{3}+\frac{1}{9}\right) \\
\leq & \alpha V^{\mathrm{i}}\left(\frac{1}{2}+\frac{1}{2}\right)+(1-\alpha) V^{\mathrm{s}}\left(\frac{1}{2}+\frac{1}{2}\right),
\end{aligned}
$$


in case $(e)$, the center agent would get payoff

$$
\begin{aligned}
& \alpha V^{\mathrm{i}}\left(\frac{1}{3}+\frac{1}{3}+\frac{1}{6}+\frac{1}{24}+\frac{1}{48}\right)+(1-\alpha) V^{\mathrm{s}}\left(\frac{1}{3}+\frac{1}{3}+\frac{1}{6}\right) \\
< & \alpha V^{\mathrm{i}}\left(\frac{1}{2}+\frac{1}{2}\right)+(1-\alpha) V^{\mathrm{s}}\left(\frac{1}{2}+\frac{1}{2}\right),
\end{aligned}
$$

and in case $(f)$, a periphery agent would get payoff

$$
\begin{aligned}
& \alpha V^{\mathrm{i}}\left(\frac{1}{4}+\frac{1}{4}+\frac{1}{8}+\frac{1}{16}+\frac{1}{32}\right)+(1-\alpha) V^{\mathrm{s}}\left(\frac{1}{4}+\frac{1}{4}\right) \\
\leq & \alpha V^{\mathrm{i}}\left(\frac{1}{2}+\frac{1}{4}\right)+(1-\alpha) V^{\mathrm{s}} \frac{1}{2} .
\end{aligned}
$$

$(\Longrightarrow)$ For this part of the proof, we need some extra notation. The payoff function in (2.8) can be rewritten as

$$
\Pi_{i}(g)=\frac{1}{\mu_{i}(g)} \sum_{j \in N_{i}(g)} T_{i, j}(g),
$$

where $T_{i, j}(g)$ is the total payoff that $j$ transmits to $i$ via her direct link with i. Formally,

$$
\begin{aligned}
T_{i, j}(g)= & \alpha\left(\sum_{\left(j^{\prime} \in \bar{N}_{j}(g) \backslash\{i\}\right)} \sum_{\left(p \in \mathcal{P}_{j, j^{\prime}}(g): i \notin \breve{P}\right)}^{\frac{V^{\mathrm{i}}}{\mu_{j}(g)}}+\frac{V^{\mathrm{i}}}{\mu_{j^{\prime}}(g) \cdot\left(\mu_{j}(g)\right)^{2} \cdot \prod_{k \in \breve{P}}\left(\mu_{k}(g)\right)^{2}}\right) \\
& +(1-\alpha) \frac{V^{\mathrm{s}}}{\mu_{j}(g)} .
\end{aligned}
$$

Assume that $g$ is a pairwise stable structure. Let $i$ be an agent in $g$ and $k \in N_{i}(g)$ be such that

$$
T_{i, k}(g)=\min _{j \in N_{i}(g)} T_{i, j}(g)
$$


Suppose that there exists an agent $\ell \in N_{i}(g)$ for whom it holds that

$$
T_{i, \ell}(g)>T_{i, k}(g)
$$

Deleting the link between $i$ and $k$ results in structure $g^{\prime}$, where it holds that

$$
T_{i, j}\left(g^{\prime}\right) \geq T_{i, j}(g), \forall j \in N_{i}\left(g^{\prime}\right)
$$

since $k$, to whom $j$ might be (in)directly linked, has one costly direct link less, so more informational value might flow from $j$ to $i$ via $k$. The payoff for $i$ then becomes

$$
\begin{aligned}
\Pi_{i}\left(g^{\prime}\right) & =\frac{1}{\mu_{i}(g)-1} \sum_{j \in N_{i}\left(g^{\prime}\right)} T_{i, j}\left(g^{\prime}\right) \\
& >\frac{1}{\mu_{i}(g)} \sum_{j \in N_{i}(g)} T_{i, j}(g)=\Pi_{i}(g),
\end{aligned}
$$

which contradicts pairwise stability of $g$. It follows that

$$
T_{i, j}(g)=T_{i, j^{\prime}}(g), \forall j, j^{\prime} \in N_{i}(g) .
$$

Next, suppose that $g$ contains a cycle, meaning that there exists a sequence of agents $k_{1}, \ldots, k_{n} \in N$ for whom it holds that $g_{k_{1}, k_{2}}=g_{k_{2}, k_{3}}=\ldots=$ $g_{k_{n-1}, k_{n}}=g_{k_{n}, k_{1}}=1$. Let $i$ be an agent in this cycle. Deleting the link with one of $i$ 's neighbors in the cycle, say $k$, results in $g^{\prime}$, where it holds for the other neighbor of $i$ in the cycle, say $m$, that

$$
T_{i, m}\left(g^{\prime}\right)>T_{i, m}(g),
$$

since $k$, to whom $m$ is (in)directly linked, has one costly direct link less, so more informational value flows from $k$ to $i$ via $m$. Moreover,

$$
T_{i, j}\left(g^{\prime}\right) \geq T_{i, j}(g), \forall j \in N_{i}\left(g^{\prime}\right) .
$$


The payoff for $i$ then becomes

$$
\begin{aligned}
\Pi_{i}\left(g^{\prime}\right) & =\frac{1}{\mu_{i}(g)-1} \sum_{j \in N_{i}\left(g^{\prime}\right)} T_{i, j}\left(g^{\prime}\right) \\
& >\frac{1}{\mu_{i}(g)-1} \sum_{j \in N_{i}\left(g^{\prime}\right)} T_{i, j}(g) \\
& =\frac{1}{\mu_{i}(g)} \sum_{j \in N_{i}(g)} T_{i, j}(g)=\Pi_{i}(g),
\end{aligned}
$$

where the second equality follows from equation (2.9). This implies that $g$ is not pairwise stable, leading to a contradiction. We have therefore shown that $g$ does not contain any cycle.

Suppose that $g$ consists of components that are not stars. Since we have already shown that $g$ contains no cycles, by definition all components of $g$ are trees. In a tree the number of links is one less than the number of agents. Moreover, in a tree there is a unique path between any two agents. A tree that is not a star contains an agent, say $i$, with a neighbor $h$ that only has $i$ as a neighbor, and, moreover, $i$ is directly linked to an agent $j$ who has another neighbor different from $i$. According to equation (2.9) it holds that

$$
T_{i, h}(g)=T_{i, j}(g)
$$

Since $h$ has only one neighbor, $i$, it follows that

$$
T_{i, h}(g)=\alpha V^{\mathrm{i}}+(1-\alpha) V^{\mathrm{s}}
$$

We now evaluate $T_{i, j}(g)$ and show it is smaller than $T_{i, h}(g)$. Think of $\bar{N}_{h}(g)$ as a tree with $h$ as top agent. For players $k, k^{\prime} \in \bar{N}_{h}(g), k \neq k^{\prime}$, player $k^{\prime}$ is a subordinate of $k$, denoted $k^{\prime} \in \bar{S}(k)$, if $k$ is on the unique path from $h$ to $k^{\prime}$. Player $k^{\prime}$ is a direct subordinate of $k$, denoted $k^{\prime} \in S(k)$, if $k^{\prime}$ is a subordinate of $k$ and there is a link between $k$ and $k^{\prime}$. We write

$$
T_{i, j}(g)=\alpha T_{i, j}^{\mathrm{i}}(g)+(1-\alpha) T_{i, j}^{\mathrm{s}}(g),
$$


where

$$
T_{i, j}^{\mathrm{s}}(g)=\frac{V^{\mathrm{s}}}{\mu_{j}(g)} \leq \frac{V^{\mathrm{s}}}{2}
$$

and

$$
T_{i, j}^{\mathrm{i}}(g)=\frac{V^{\mathrm{i}}}{\mu_{j}(g)}+\sum_{k \in \bar{S}(j)} \frac{V^{\mathrm{i}}}{\mu_{k}(g)\left(\mu_{j}(g)\right)^{2} \prod_{k^{\prime} \in \breve{P}_{j, k}}\left(\mu_{k^{\prime}}(g)\right)^{2}},
$$

where $p_{j, k}$ is the unique path between $j$ and $k$.

Consider $k \in \bar{S}(i)$. We define the informational payoff that $k$ receives from its subordinates by

$$
U_{k}^{\mathrm{i}}(g)=\frac{1}{\mu_{k}(g)} \sum_{k^{\prime} \in S(k)} T_{k, k^{\prime}}^{\mathrm{i}}(g)
$$

where $T_{k, k^{\prime}}^{\mathrm{i}}(g)$ is defined analogously to $T_{i, j}^{\mathrm{i}}(g)$. We obtain a recursive relation by observing that

$$
T_{k, k^{\prime}}^{\mathrm{i}}(g)=\frac{V^{\mathrm{i}}+U_{k^{\prime}}^{\mathrm{i}}(g)}{\mu_{k^{\prime}}(g)}
$$

We show by induction that

$$
U_{k}^{\mathrm{i}}(g) \leq V^{\mathrm{i}}\left(\mu_{k}(g)-1\right)
$$

from which it follows that

$$
T_{k, k^{\prime}}^{\mathrm{i}}(g) \leq \frac{V^{\mathrm{i}}+V^{\mathrm{i}}\left(\mu_{k^{\prime}}(g)-1\right)}{\mu_{k^{\prime}}(g)}=V^{\mathrm{i}},
$$

and, consequently,

$$
T_{i, j}^{\mathrm{i}}(g) \leq V^{\mathrm{i}}
$$

Let $K^{0}$ be the set of agents without subordinates. For $m \geq 1$, let $K^{m}$ be the set of agents with all subordinates in $K^{0} \cup \cdots \cup K^{m-1}$. Let $m^{\prime}$ be the smallest integer for which $j \in K^{m^{\prime}}$. First consider an agent $k$ in $K^{0}$, the set of agents without subordinates. Then $U_{k}^{\mathrm{i}}(g)=0=V^{\mathrm{i}}\left(\mu_{k}(g)-1\right)$, so $(2.11)$ is satisfied. 
Suppose that (2.11) holds for agents in $K^{m}, m<m^{\prime}$. Consider an agent $k \in K^{m+1}$.

$$
\begin{aligned}
U_{k}^{\mathrm{i}}(g) & =\frac{1}{\mu_{k}(g)} \sum_{k^{\prime} \in S(k)} T_{k, k^{\prime}}^{\mathrm{i}}(g) \\
& \leq \frac{1}{\mu_{k}(g)} \sum_{k^{\prime} \in S(k)}\left(\frac{V^{\mathrm{i}}+V^{\mathrm{i}}\left(\mu_{k^{\prime}}(g)-1\right)}{\mu_{k^{\prime}}(g)}\right) \\
& =\frac{\mu_{k}(g)-1}{\mu_{k}(g)} V^{\mathrm{i}} \leq \frac{1}{2} V^{\mathrm{i}}\left(\mu_{k}(g)-1\right),
\end{aligned}
$$

so (2.11) holds for all $k \in \bar{S}(i)$.

Combining (2.10) and (2.12) implies $T_{i, j}(g)<T_{i, h}(g)$, a contradiction to equation (2.9), so $g$ consists of star components only.

Lemma 14 implies that these stars have at most three agents. "Stars" of a single agent cannot be part of $g$, for it is always strictly beneficial for this single agent to create a link to the center agent of another star, whereas this center agent is indifferent or improves if she is isolated too.

Table 2.1 pictures all structures thus proven to be pairwise stable in the case with both social and informational value from communication for $n \leq 6$.

Comparing these results to the purely social value case, clearly a smaller range of very fragmented structures turns out to be pairwise stable in the mixed case where transferable informational value also plays a role. Specifically, even with $\alpha$ slightly above zero, regular structures are never pairwise stable and also the example structures in Figures 2.1, 2.2, and 2.3 are not stable anymore. This may seem counter-intuitive, since apparently transferability of informational value causes structures to become more fragmented and therefore less able to transfer information.

The intuition behind this finding is that the link specificity property of communication is now strong enough to prevent individuals from maintaining many links, because it is strengthened by the transferability of value. 


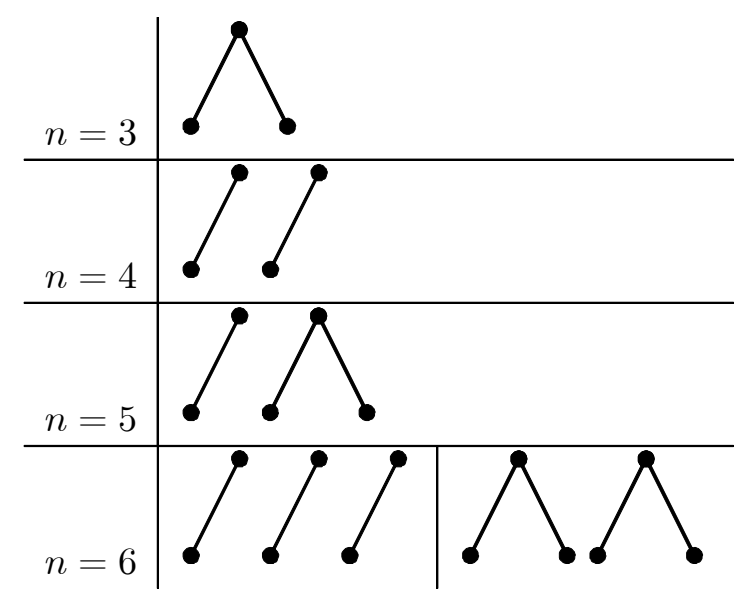

Table 2.1: Pairwise Stable Structures With Informational Value

For example, in a wheel structure of three agents, an agent cannot improve (or decrease) her social payoff by deleting one of her links, but she can improve her informational payoff:

$$
\frac{V^{\mathrm{i}}}{2}+\frac{V^{\mathrm{i}}}{4}>\frac{2 V^{\mathrm{i}}}{4}+\frac{2 V^{\mathrm{i}}}{16} .
$$

The co-author model of Jackson and Wolinsky (1996) also contained a type of link specificity, but since it was not combined with value transferability, the resulting stable structures were not as fragmented. Similarly, the connections model of Jackson and Wolinsky contained value transferability, but since it was not combined with link specificity, the resulting structures are not fragmented at all.

Likewise, most studies reveal less fragmented stable structures, e.g. Goyal \& Vega-Redondo (2004) find large star structures in their setting of structural holes. Therefore, our model can explain real-life phenomena like the evolvement of threads in online communities into strong reciprocal ties (Fisher et al. 2006). 


\subsection{Welfare}

In this section, the structural results from the previous sections are assessed by their impact on welfare. The welfare of a network structure is defined as the sum of payoffs for all agents. ${ }^{6}$ The best and worst pairwise stable network structures from a welfare point of view are characterized (Sections 2.4.1 and 2.4.2). It turns out that the purely social value situation performs badly in the worst-case scenario, but that this usual tension between stability and efficiency (e.g., Jackson and Wolinsky 1996) is largely resolved in the mixed situation with both social and informational value. We also identify differences in welfare due to variations in social versus informational orientation in the network (Section 2.4.3).

Definition 16 (welfare) The welfare provided by structure $g$ is given by

$$
W(g)=\sum_{i \in N} \Pi_{i}(g) .
$$

Definition 17 (efficiency) The structure $g$ is more efficient than the structure $g^{\prime}$ if it holds that

$$
W(g)>W\left(g^{\prime}\right)
$$

\subsubsection{Best Pairwise Stable Structures}

The next proposition describes the most efficient pairwise stable structures.

Proposition 18 If $n$ is even, the most efficient pairwise stable structures consist of pairs only. If $n$ is odd, the most efficient pairwise stable structures consist of one three-agent star and furthermore only pairs.

\footnotetext{
${ }^{6}$ Alternatively, it may be interesting from a managerial point of view, to study the structural effects on the actual product-, service-, or firm-related information exchanged, thus disregarding the value derived from the social aspects of communication. However, this does not cause results to differ considerably.
} 
Proof. In the case with only social value $(\alpha=0)$,

$$
\Pi_{i}(g)=\frac{V^{\mathrm{s}}}{\mu_{i}(g)} \sum_{j \in N_{i}(g)} \frac{1}{\mu_{j}(g)},
$$

the highest possible payoff for player $i$ is obtained if $\mu_{j}(g)=1$ for all $j \in$ $N_{i}(g)$. If $i$ belongs to a pair, then this condition holds, so a player in a pair obtains the highest payoff possible. The highest payoff possible is therefore equal to $V^{\mathrm{s}}$. If a structure consists of only pairs, then all players get the highest payoff possible, and as a consequence, also the welfare provided by the structure is maximized.

If $n$ is odd, then the structure cannot consist of pairs only. There should be at least one player, say $i$, with at least two neighbors. Since pairwise stable structures are equal neighbor degree structures, all neighbors of neighbors of $i$ have the same degree as $i$, and therefore $i$ 's neighbors have a payoff equal to $V^{\mathrm{s}}$ divided by $\mu_{i}(g)$. The sum of the payoffs of all players is therefore maximized if there is only one player with more than one neighbor, and this player should have two neighbors. The structure therefore consists of one three-agent star and furthermore only pairs.

In the case with both social and informational value $(0<\alpha<1)$, Proposition 15 implies that all pairwise stable structures consist of disjoint star components of two or three agents. The payoff for an agent in a pair is

$$
\alpha V^{\mathrm{i}}+(1-\alpha) V^{\mathrm{s}}
$$

whereas the average payoff for an agent in a three-agent star is only

$$
\frac{5}{6} \alpha V^{\mathrm{i}}+\frac{2}{3}(1-\alpha) V^{\mathrm{s}}
$$

Welfare of the structure is maximized by having only pairs if $n$ is even, and one three-agent star and furthermore only pairs if $n$ is odd. 
It is not hard to show that in the social value case, the structures identified in Proposition 18 are moreover the most efficient ones among all structures, pairwise stable or not. This conclusion does not differ when informational value is included in the analysis. Agents that are part of a pair obtain the highest payoff possible, $\alpha V^{\mathrm{i}}+(1-\alpha) V^{\mathrm{s}}$, so structures with many pairs achieve a high degree of efficiency.

The most efficient pairwise stable structures coincide in the purely social value case and the case with both social and informational value. The worst-case scenarios, i.e. the least efficient pairwise stable structures, are considerably different between these cases, as is shown in the next subsection.

\subsubsection{Worst Pairwise Stable Structures}

In the social value situation, many pairwise stable structures exist that are not efficient. The worst-case scenario is described in the following proposition.

Proposition 19 In the social value case, the complete structure provides the lowest welfare of all pairwise stable structures.

Proof. Since

$$
\Pi_{i}(g)=\frac{V^{\mathrm{s}}}{\mu_{i}(g)} \sum_{j \in N_{i}(g)} \frac{1}{\mu_{j}(g)},
$$

and since every player has at least one link in a pairwise stable structure, the lowest payoff possible for player $i$ is obtained if all players $j \in N_{i}(g)$ have maximal degree $\mu_{j}(g)=n-1$. It follows that the complete structure has the lowest welfare of all pairwise stable structures.

The worst-case scenarios for the situation with both social and informational value are described in the following proposition. 
Proposition 20 In the case with both social and informational value,

(a) if $n \in\{3,6,9, \ldots\}$, a structure consisting of only three-agent stars provides the lowest welfare of all pairwise stable structures,

(b) if $n \in\{4,7,10, \ldots\}$, a structure consisting of two pairs and furthermore only three-agent stars provides the lowest welfare of all pairwise stable structures, ${ }^{7}$

(c) if $n \in\{5,8,11, \ldots\}$, a structure consisting of one pair and furthermore only three-agent stars provides the lowest welfare of all pairwise stable structures.

Proof. Proposition 15 implies that all pairwise stable structures consist of disjoint star components of two or three agents. The payoff for an agent in a pair is

$$
\alpha V^{\mathrm{i}}+(1-\alpha) V^{\mathrm{s}}
$$

whereas the average payoff for an agent in a three-agent star is only

$$
\frac{5}{6} \alpha V^{\mathrm{i}}+\frac{2}{3}(1-\alpha) V^{\mathrm{s}}
$$

which implies (a), (b), and (c).

Note that the worst-case scenarios in the situation with both social and informational value are not as bad as the worst-case scenario in the social value case. For any value of $n, V^{\mathrm{i}}$, and $V^{\mathrm{s}}$, the least efficient pairwise stable structure achieves an efficiency of at least $2 / 3$ times the most efficient structure. In the social value case, for any value of $V^{\mathrm{s}}$, this ratio can be as bad as $\frac{1}{n-1}$, which tends to zero as $n$ grows large. Therefore, the usual tension between stability and efficiency (e.g., Jackson and Wolinsky 1996) can largely be resolved by introducing value transferability into a setting with link specificity.

\footnotetext{
${ }^{7}$ For $n=4$ this implies a structure consisting of two pairs only.
} 


\subsubsection{Informational versus Social Orientation}

Apparently, there are welfare differences attached to structural differences in an OCCN and structural differences arise by its informational versus social orientation $(\alpha)$. A managerial implication is that the welfare level provided by an OCCN can be influenced depending on how strongly $\alpha$ can be influenced. In order to affect $\alpha$, communication network managers could think of modifying moderator tasks, entry policy, rules of conduct, and reward systems, hereby emphasizing social versus informational value. The question whether more emphasis on social aspects rather than informational aspects is required in maximizing welfare, depends on the ratio between $V^{\mathrm{s}}$ and $V^{\mathrm{i}}$. We denote this ratio by $x$ :

$$
V^{\mathrm{s}}=x V^{\mathrm{i}}
$$

For $n=10$ and three values for $x$ the impact of $\alpha$ on welfare is illustrated in Figure 2.4, where the top line each time indicates the welfare for the most efficient pairwise stable structures and the bottom line for the least efficient ones. More generally, we can prove the following two propositions.

Proposition 21 The worst-case scenario for social value only $(\alpha=0)$ provides lower welfare than a worst-case scenario where also a marginal amount of transferable informational value is provided $(\alpha \downarrow 0){ }^{8}$

Proof. For $\alpha=0$, Proposition 19 shows that the complete structure is the worst-case scenario, which provides a total payoff of

$$
\frac{n}{n-1} V^{\mathrm{s}}
$$

${ }^{8}$ Note that results for the informational case $(\alpha=1)$ can be derived analogously. 


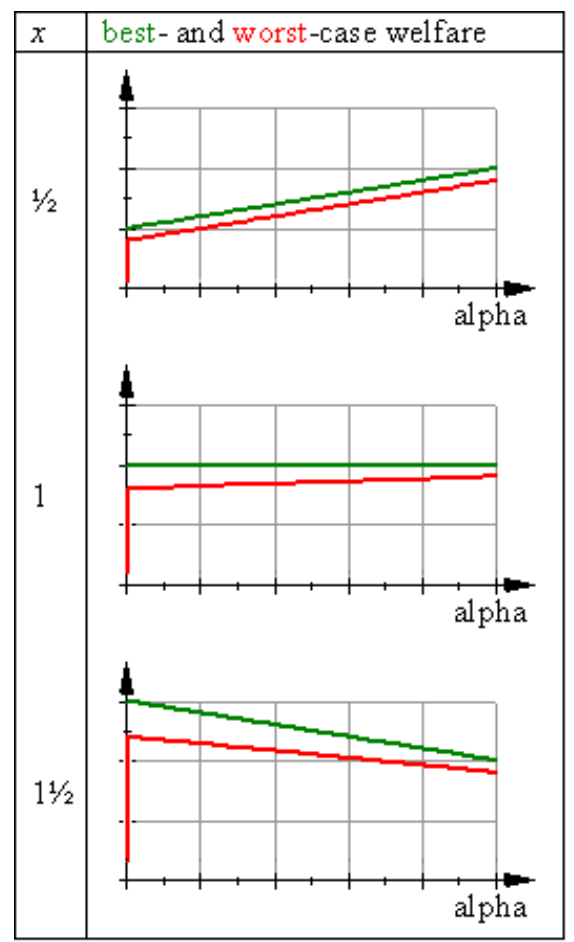

Figure 2.4: Impact of $\alpha$ on Welfare Illustrated for $n=10$

For $0<\alpha<1$, Proposition 20 shows that the total payoff in the worst-case scenario is at least equal to

$$
n\left(\frac{5}{6} \alpha V^{\mathrm{i}}+\frac{2}{3}(1-\alpha) V^{\mathrm{s}}\right) \rightarrow n \frac{2}{3} V^{\mathrm{s}} \text { if } \alpha \downarrow 0 .
$$

It holds that

$$
n \frac{2}{3} V^{\mathrm{s}}>\frac{n}{n-1} V^{\mathrm{s}},
$$

since $n \geq 3$.

Proposition 22 In the case with both social and informational value,

(a) the higher $\alpha$ the better the best-case as well as the worst-case scenarios if $x<1$, and 
(b) the lower $\alpha$ the better the best-case as well as the worst-case scenarios if $x>1 \frac{1}{4} \cdot{ }^{9}$

Proof. If $n$ is even, Proposition 18 implies that a structure consisting of only pairs is a best-case scenario, which provides a total payoff of

$$
V^{\mathrm{i}} n((1-x) \alpha+x)
$$

which is increasing in $\alpha$ if $x<1$ and decreasing in $\alpha$ if $x>1 \frac{1}{4}$. If $n$ is odd, Proposition 18 implies that a structure consisting of one three-agent star and furthermore only pairs is a best-case scenario, which provides a total payoff of

$$
V^{i}\left(\left(n-\frac{1}{2}-(n-1) x\right) \alpha+(n-1) x\right)
$$

which, since $n \geq 3$, is increasing in $\alpha$ if $x<1$ and decreasing in $\alpha$ if $x>1 \frac{1}{4}$.

If $n=3,6,9, \ldots$ Proposition 20 implies that a structure consisting of only three-agent stars is a worst-case scenario, which provides a total payoff of

$$
V^{i} n\left(\left(\frac{5}{6}-\frac{2}{3} x\right) \alpha+\frac{2}{3} x\right)
$$

which is increasing in $\alpha$ if $x<1$ and decreasing in $\alpha$ if $x>1 \frac{1}{4}$. If $n=$ $4,7,10, \ldots$, Proposition 20 implies that a structure consisting of two pairs and furthermore only three-agent stars is a worst-case scenario, which provides a total payoff of

$$
V^{i}\left(\left(\frac{5}{6} n+\frac{2}{3}-\left(\frac{2}{3} n+1 \frac{1}{3}\right) x\right) \alpha+\left(\frac{2}{3} n+1 \frac{1}{3}\right) x\right),
$$

which, since $n \geq 4$, is increasing in $\alpha$ if $x<1$ and decreasing in $\alpha$ if $x>1 \frac{1}{4}$. The proof for $n=5,8,11, \ldots$ is similar.

\footnotetext{
${ }^{9}$ For $x$ between 1 and $1 \frac{1}{4}$, comparative statics depend on the value of $n$.
} 


\subsection{Discussion}

This chapter has shown that the structure of bilateral communication links within OCCNs can be appropriately studied using a model based on the game-theoretic literature of social and economic network formation. A combination of important aspects common to OCCNs was incorporated that had not been investigated until now: the negative externality of link specificity and the positive externality of informational value transferability.

In the case of communication having social value only, illustrating the separate impact of link specificity on structure, the set of pairwise stable structures was characterized and shown to include a wide range of nonstandard architectures like highly connected and "small world" structures, whereas previous models for social and economic network formation mostly predicted simple architectures like stars and wheels.

In the case of communication from which both social and informational value is derived, illustrating the joint impact of link specificity and value transferability on structure, only structures that consist of disjoint star components of two or three agents were shown to be pairwise stable. Herewith, we predict much more fragmentation than usually in the literature about social and economic network formation, where mostly only one of these two features was included.

From a welfare point of view, a mix of social and informational focus was shown to be better than a purely social focus. This illustrates that the fragmentation effect arising from the combination of link specificity and value transferability decreases the common tension between stability and efficiency. Furthermore, guidelines were provided for when it is better to stimulate either the social or the informational orientation of an OCCN as a managerial concern. For example, a Saab representative could stimulate the informational focus of the Saab network by introducing a reward system that allows users to grade the usefulness of fellow users' posts, or the social focus by appointing moderators who inspire the exchange of personal experiences. 
Further research could study the sets of pairwise stable and efficient network structures with other functional forms to model the link specificity property of communication. In this chapter it is modeled such that value is divided proportionally by the number of links that agents face, whereas other link specificity forms might be applicable to other communication forms. For example, agents may have economies of scale in coping with several links or value derived from different agents may be complementary, which would diminish the fragmentation effect as caused by the current form of link specificity.

The informational versus social orientation is of managerial concern here. The model can be extended to allow for investigating the effectiveness of other community design issues, like introducing an expert or a social animator in the OCCN, who possibly does not even act strategically in the link formation game. An example would be Dr. Love in the community studied by Holme et al. (2004). Therefore, future research could introduce valuation heterogeneity in the sense that agents represent different values for their fellow customers or have different opinions on the values of their fellow customers (e.g., Galeotti et al. 2006).

Another extension of the current model could be to relax the assumption that agents divide their available effort equally among all their relationships, thus entering the subject of link quality and dropping the common 1-0 formulation of links. As suggested by Goyal (2005), a first step into this direction would be to introduce a distinction between strong links in which both agents actively interact with each other, and weak links in which one agent is active and the other is not, where the passive agent can only access the direct value from her active partner.

Besides, a possible follow-up would be to empirically examine the applicability of the used payoff function in diverse contexts. The model could be tested experimentally, contributing to an emerging literature as surveyed by Kosfeld (2004). Accordingly, we hope that our current work stimulates future research in the appealing area of OCCNs and the role of balancing social and informational value in these communication networks. 


\section{Chapter 3}

\section{The Strategic Aspect: A Simulation Approach}

We study the structure and efficiency of bilateral communication links by a game-theoretic model of network formation. This enhances the model as introduced in Chapter 2 in several respects. First, link specificity - meaning that the more direct links somebody has to maintain with others, the less she is able to specify her attention per link within her time available, so that the value of her links decreases - is no longer fixed at a high level. Second, valuation heterogeneity is introduced, implying that agents represent different values for each other. We simulate pairwise stable networks for different levels of link specificity, focus on informational versus social value from communication, and valuation heterogeneity. It is illustrated that their structure properties heavily depend on these parameter values, which results in efficiency and equity differences among settings. Accordingly, we assess the effectivity of several managerial tools for common communication networks. 


\subsection{Introduction}

With this dissertation we study the structure and efficiency of bilateral communication links by a game-theoretic model of network formation in which individuals choose to create and maintain links if the participants in the link benefit from doing so, which results in a pairwise stable network structure (Jackson and Wolinsky 1996). In order to enlarge the model's scope of application as introduced in Chapter 2 to a broader range of communication settings as well as to increase the model's managerial relevance, this chapter performs simulation studies for the model enhanced with two suggestions for further research (see Section 2.5).

First, an important property of communication is link specificity (e.g., Currarini 2007), meaning that having to maintain more direct links leads to somebody's being less able to specify her attention per link within her time available, so that the value of her links decreases. In Chapter 2 this was modeled such that value is divided proportionally by the number of links that agents face, whereas other functional forms might be applicable to other communication settings: e.g., agents may have economies of scale in coping with several links or value derived from different agents may be complementary. Therefore, in the current chapter the extent of link specificity is varied and thus no longer fixed at this high level.

Second, this chapter introduces valuation heterogeneity in the sense that agents may represent different values for each other. Consequently, the common simplifying assumption of agent homogeneity is relaxed (e.g., Galeotti et al. 2006). In particular, we explore four relevant patterns of valuation: homogeneity and expertise heterogeneity, each with and without the presence of one especially highly valued expert.

We simulate pairwise stable networks for three levels of link specificity, three levels of focus on informational versus social value from communication, and four patterns of valuation heterogeneity. Structure and payoff properties of these networks are described and used to assess the effectivity of several managerial tools for common communication networks. 
The chapter is structured as follows. In Section 3.2 we outline the network formation model including the more general payoff function allowing for variable link specificity and heterogeneity. Section 3.3 describes the simulation procedure for this model, Section 3.4 gives corresponding definitions, and Section 3.5 provides its results. The subsequent effectivity of management tools as well as suggestions for further research are discussed in Section 3.6.

\subsection{Model}

The communication network formation model as introduced in Chapter 2 is extended by variable link specificity and heterogeneity as follows. Consider a community of agents $N=\{1, \ldots, n\}, n \geq 3$. A direct link $g_{i, j}$ between agents $i$ and $j$ in this community $(i, j \in N ; i \neq j)$ can be interpreted as an undirected virtual communication relationship between $i$ and $j$ : for any two agents $i$ and $j, g_{i, j}=g_{j, i}$, and $g_{i, i}=0$. The total payoff for agent $i$ in link structure $g$ is given by

$$
\Pi_{i}(g)=\left\{\begin{array}{cl}
\alpha \sum_{j \in \bar{N}_{i}(g)} \sum_{p \in \mathcal{P}_{i, j}(g)} \frac{V_{i, j}^{\mathrm{i}}}{\left(\mu_{i}(g) \cdot \prod_{k \in \breve{P}}\left(\mu_{k}(g)\right)^{2} \cdot \mu_{j}(g)\right)^{\rho}} & \text { if } \quad \mu_{i}(g)>0 \\
+(1-\alpha) \sum_{j \in N_{i}(g) \frac{V_{i, j}^{\mathrm{s}}}{\left(\mu_{i}(g) \cdot \mu_{j}(g)\right)^{\rho}}} & \text { if } \mu_{i}(g)=0,
\end{array}\right.
$$

where $\alpha$ is the proportion of communication through each link in the community that concerns product-, service- or firm-related information and $1-\alpha$ is the proportion of communication through each link in the community that concerns social interaction; $N_{i}(g)$ is the set of agents with whom $i$ has a direct link, where agent $j$ is a neighbor of agent $i$ if $j \in N_{i}(g)$, and $\mu_{i}(g)=\left|N_{i}(g)\right|$ is the number of neighbors of agent $i$, which can also be referred to as the degree of $i$, and $\bar{N}_{i}(g)$ is the set of agents with whom $i$ has either a direct or an indirect link; $\mathcal{P}_{i, j}(g)$ is the set of paths between $i$ and $j$, where a path is defined as a sequence of consecutive edges without repeated 
nodes, and $\breve{P}$ is the set of agents on path $p$ between $i$ and $j$ excluding $i$ and $j$ themselves; $V_{i, j}^{\mathrm{i}}>0$ denotes the informational value that $i$ would derive from communication with $j$ if neither $i$ nor $j$ were linked to any other agent and interaction would only have informational value, and $V_{i, j}^{\mathrm{s}}>0$ denotes the social value that $i$ would derive from communication with $j$ if neither $i$ nor $j$ were linked to any other agent and interaction would only have social value; and $\rho$ indicates the level of link specificity.

Observe that the contributions of two agents in their bilateral process of communication value creation are reflected by a Cobb-Douglas production function with both output elasticities equal to $\rho$, which was assumed to equal 1 in Chapter 2. Also, in that chapter it was assumed that $V_{i, j}^{\mathrm{i}}=V^{\mathrm{i}}$ and $V_{i, j}^{\mathrm{s}}=V^{\mathrm{s}}$ for all $i, j \in N$. For the more general model thus described, stable network structures are again predicted to emerge by using the concept of pairwise stability (Jackson and Wolinsky 1996).

\subsection{Method}

The model is simulated as follows. A simulation starts with a random network in the sense that for every $i, j \in N ; i \neq j, g_{i, j}$ is randomly chosen to equal 0 or 1 . In every iteration the program randomly determines whether there will be an attempt to delete or create a link.

If for an iteration this turns out to be delete, one agent is randomly drawn from the community and subsequently another one. If there exists a link between these two agents, the first agent calculates the payoff she will earn when she deletes this link (equation 3.1). If this is strictly higher than the payoff she earns with the current network, the deletion is performed. If no link exists between the two agents or the first agent does not gain by its deletion, the current network is maintained until the next iteration.

If the attempt of the iteration appears to be create, two agents are randomly drawn from the community. If no link exists between these two agents, they both calculate the payoff they will earn when they create such 
a link. If this is weakly higher for both agents and strictly higher for at least one of them, the creation is performed. If there already exists a link between these two agents or one of them looses or none of them gains by its creation, the current network is maintained.

In this way, 10,000 iterations are performed consecutively. Afterwards in the same simulation another 1,000 iterations are executed for which the networks are stored. If these 1,000 networks are the same, it is assumed that the simulation converged to a pairwise stable network. The program performs 100 of these simulations for each of $3\left(\rho=\frac{1}{2}, \frac{3}{4}, 1\right)^{1} \times 3\left(\alpha=0, \frac{1}{2}, 1\right)$ x 4 (see Section 3.4) parameter value combinations.

Subsequently, to compare outcomes to a situation in which agents hold social preferences, for each of these settings the procedure is repeated, assuming that agents - instead of their personal payoff - hold welfare as their objective function:

$$
W(g)=\sum_{i=1}^{n} \Pi_{i}(g) .
$$

Pairwise stability in such a simulation - again assumed if the last 1,000 iteration outcomes are the same network - can be used to create a meaningful measure of local efficiency.

Definition 23 The structure $g$ is locally efficient if for all $i, j \in N$ with $g_{i, j}=1$ it holds that

$$
W(g) \geq W\left(g^{\prime}\right),
$$

where $g^{\prime}$ is such that $g_{i, j}^{\prime}=0$ and $g_{k, l}^{\prime}=g_{k, l}$ for all $\{k, l\} \neq\{i, j\}$, and for all $i, j \in N$ with $g_{i, j}=0$ it holds that

$$
W(g) \geq W\left(g^{\prime}\right)
$$

where $g^{\prime}$ is such that $g_{i, j}^{\prime}=1$ and $g_{k, l}^{\prime}=g_{k, l}$ for all $\{k, l\} \neq\{i, j\}$.

Community size $n=6$ is chosen for all simulations, since it is large

\footnotetext{
${ }^{1}$ Notice that $\varrho=\frac{1}{2}$ coincides with constant returns to scale in the Cobb-Douglas function reflecting agents' contributions in the bilateral production of communication.
} 
enough to illustrate interesting tendencies as well as small enough to generate reasonable calculation times regarding the exponentially increasing number of paths in the payoff function (equation 3.1). The tendencies in the results are robust for the odd number of agents $n=5$.

\subsection{Definitions}

We define the four valuation patterns for which we run simulations and the structure measures we use to compare their outcomes.

\subsubsection{Valuation Patterns}

The first pattern of valuation to consider is homogeneity, which we define as follows:

$$
V_{i, j}^{\mathrm{i}}=V_{i, j}^{\mathrm{s}}=6 \forall i, j \in N: i \neq j
$$

Next, we study a valuation pattern that is heterogeneous in the sense that all agents are equally valued except for one, who is much higher valued, both socially and informationally. This pattern is based on the management tool of adding an expert to the community. The valuations in the case that agent 1 is an expert are given by

$$
\begin{aligned}
& V_{i, 1}^{\mathrm{i}}=V_{i, 1}^{\mathrm{s}}=21 \forall i \in N: i \neq 1, \\
& V_{i, j}^{\mathrm{i}}=V_{i, j}^{\mathrm{s}}=3 \forall i, j \in N: i \neq j, j \neq 1 .
\end{aligned}
$$

These values are such that the sum of valuations over the community of 6 agents is equal between this case and the completely homogeneous case. Therefore, in the different valuation patterns the complete network provides the same welfare given $\rho$ and $\alpha$ (e.g., 90.37 for $\rho=\frac{1}{2}, \alpha=1$ ). Of course, the empty network provides the same welfare in all parameter conditions (i.e., $0)$.

Then, a valuation pattern is studied in which all agents can be ranked 
according to a gradually decreasing value they represent for others, both socially and informationally. Expertise heterogeneity is defined as

$$
\begin{aligned}
V_{i, 1}^{\mathrm{i}} & =V_{i, 1}^{\mathrm{s}}=11 \forall i \in N: i \neq 1, \\
V_{i, 2}^{\mathrm{i}} & =V_{i, 2}^{\mathrm{s}}=9 \forall i \in N: i \neq 2, \\
V_{i, 3}^{\mathrm{i}} & =V_{i, 3}^{\mathrm{s}}=7 \forall i \in N: i \neq 3, \\
V_{i, 4}^{\mathrm{i}} & =V_{i, 4}^{\mathrm{s}}=5 \forall i \in N: i \neq 4, \\
V_{i, 5}^{\mathrm{i}} & =V_{i, 5}^{\mathrm{s}}=3 \forall i \in N: i \neq 5, \\
V_{i, 6}^{\mathrm{i}} & =V_{i, 6}^{\mathrm{s}}=1 \forall i \in N: i \neq 6 .
\end{aligned}
$$

Again these values are such that the sum of valuations over the community of 6 agents is equal among valuation patterns.

Finally, we investigate a valuation pattern in which the effects of equations 3.3 and 3.4 are combined: except for one, who is valued much higher, all agents can be ranked according to a gradually decreasing value they represent for others, both socially and informationally. To equal the sum of valuations over the community among valuation patterns, we define expertise heterogeneity with expert as

$$
\begin{aligned}
V_{i, 1}^{\mathrm{i}} & =V_{i, 1}^{\mathrm{s}}=21 \forall i \in N: i \neq 1, \\
V_{i, 2}^{\mathrm{i}} & =V_{i, 2}^{\mathrm{s}}=5 \forall i \in N: i \neq 2, \\
V_{i, 3}^{\mathrm{i}} & =V_{i, 3}^{\mathrm{s}}=4 \forall i \in N: i \neq 3, \\
V_{i, 4}^{\mathrm{i}} & =V_{i, 4}^{\mathrm{s}}=3 \forall i \in N: i \neq 4, \\
V_{i, 5}^{\mathrm{i}} & =V_{i, 5}^{\mathrm{s}}=2 \forall i \in N: i \neq 5, \\
V_{i, 6}^{\mathrm{i}} & =V_{i, 6}^{\mathrm{s}}=1 \forall i \in N: i \neq 6 .
\end{aligned}
$$




\subsubsection{Structure Measures}

In order to compare simulation outcomes among levels of $\rho$ and $\alpha$, we use the density of a structure (Wasserman and Faust 1994, p. 164):

$$
D=\frac{\sum_{i=1}^{n} \mu_{i}(g)}{n(n-1)}
$$

Our intuitive prediction is that in general, a higher level of link specificity $(\rho)$ makes communication more costly, and therefore the expected density of a simulated network is lower. Furthermore, a higher level of focus on informational value $(\alpha)$ provides more value spillovers from indirect links, and thus the expected density of a pairwise stable network is higher.

For the valuation patterns (3.3), (3.4), and (3.5), we use an additional structure variable to compare pairwise stable networks among levels of $\rho$ and $\alpha$ on an individual level: in how far an agent takes a central position relative to the other agents. Relative centrality of agent $k$ is defined as

$$
C_{k}=\frac{\left(\frac{\mu_{k}(g)}{\frac{1}{n} \sum_{i=1}^{n} \mu_{i}(g)}\right)-1}{(n-2) / 2} .
$$

This measure is adapted from standardized actor degree centrality (Wasserman and Faust 1994, p. 179) to express the centrality of an agent as relative to the centrality of the other agents in the community, where it holds that

$$
\sum_{k=1}^{n} C_{k}=1 .
$$

For example, in a complete or empty network all agents have a relative centrality equal to 0 and the center agent of the star structure with $(n-1)$ periphery agents has a relative centrality equal to 1 . 


\subsection{Results}

In this section we discuss the simulation outcomes for the parameter settings as mentioned.

\subsubsection{Convergence}

It appears that all 100 simulations in every setting converge to pairwise stable networks as described above (page 49). Therefore, we deal with structure and payoff properties of these networks.

\subsubsection{Homogeneity}

For homogeneity as defined in (3.2), the following subsections discuss structure properties of the simulated pairwise stable and locally efficient networks and then their payoff properties.

\section{Structure Properties}

For $\rho=1$, the simulation outcomes confirm the analytical results from Chapter 2. For $\alpha>0$ the pairwise stable networks consist of disjoint star components of two or three agents only whereas for $\alpha=0$ they for instance also contain wheel components. The locally efficient networks for $\rho=1$ consist of disjoint star components of two or three agents for $0 \leq \alpha \leq 1$, so not only of pairs like the most efficient pairwise stable networks from Chapter 2 . Thus, also with this local efficiency measure the tendency between efficiency and stability for $\rho=1, \alpha=0$ is validated.

For each combination of three levels of link specificity and three levels of focus on informational versus social value from communication, the average density and the standard deviation of the 100 simulated pairwise stable and locally efficient networks are given in Table 3.1. Results for local efficiency 
are indicated in Italics in separate columns in all following tables. Furthermore, in all structure tables one, two, or three stars mark a difference of pairwise stability with respect to local efficiency of at least $0.1,0.2$, or 0.3 respectively.

\begin{tabular}{|c|l|l|l|l|l|l|}
\hline$\rho \backslash \alpha$ & \multicolumn{2}{|c|}{0} & \multicolumn{2}{c|}{$\frac{1}{2}$} & \multicolumn{2}{c|}{1} \\
\hline$\frac{1}{2}$ & $1.00^{* * *}$ & 0.556 & 1.00 & 1.00 & 1.00 & 1.00 \\
& $(0.000)$ & $(0.136)$ & $(0.000)$ & $(0.000)$ & $(0.000)$ & $(0.000)$ \\
\hline$\frac{3}{4}$ & $0.887^{* * *}$ & 0.231 & $0.916^{* * *}$ & 0.222 & $0.839^{* * *}$ & 0.394 \\
& $(0.208)$ & $(0.033)$ & $(0.194)$ & $(0.031)$ & $(0.207)$ & $(0.019)$ \\
\hline 1 & 0.280 & 0.233 & 0.231 & 0.239 & 0.234 & 0.236 \\
& $(0.106)$ & $(0.033)$ & $(0.033)$ & $(0.033)$ & $(0.033)$ & $(0.035)$ \\
\hline
\end{tabular}

Table 3.1: Densities for Homogeneity

The basic intuition about the effect of $\rho$ on density is thus confirmed by the simulation outcomes, for the well-known fragmentation for $\rho=1$ (e.g., for $\alpha=\frac{1}{2}: D=0.231$ ) reverses to complete networks when $\rho$ decreases to $\frac{1}{2}(D=1.00)$. However, the intuition about the effect of $\alpha$ on density is contradicted by the simulation outcomes as it was by the analytical results of Chapter 2 for $\rho=1$, since for $\rho=\frac{3}{4}$ we find a higher density for $\alpha=\frac{1}{2}$ (0.916) than for $\alpha=1(0.839)$. This can again be ascribed to the interaction effect of value transferability and link specificity.

We see that whereas for $\rho=1$, fragmentation eliminates differences between locally efficient and pairwise stable networks (even for $\alpha=0$, the simulation process appears to select the less dense pairwise stable networks and for example no complete structures), for $\rho=\frac{1}{2}$ the opposite effect performs the same job: except for the locally efficient networks for $\alpha=0$, all locally efficient as well as pairwise stable networks are full structures. For the intermediate level of specificity $\left(\rho=\frac{3}{4}\right)$, we find large differences in density between the locally efficient and pairwise stable networks in the sense that the pairwise stable networks are more connected. The following example elaborates one of these cases.

Example 24 Let $\rho=\frac{3}{4}, \alpha=\frac{1}{2}$, then Table 3.2 provides an overview of the types and numbers of pairwise stable networks resulting from 100 simulations 


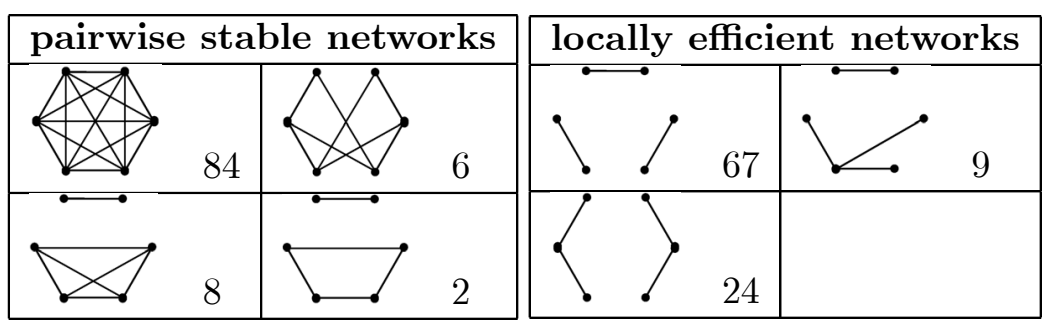

Table 3.2: Example of Structures for Homogeneity

and the types and numbers of locally efficient networks resulting from another 100 simulations.

Thus, for this intermediate level of $\rho$, the pairwise stable networks are highly connected as with lower levels of link specificity, whereas the locally efficient networks are very fragmented as with higher levels of link specificity.

\section{Payoff Properties}

As a means to investigate in how far the structure differences between the locally efficient and pairwise stable networks actually lead to welfare differences, for each parameter setting, the average welfare and the standard deviation of the simulated pairwise stable and locally efficient networks are given in Table 3.3. Furthermore, in all payoff tables one, two, or three stars mark a difference of pairwise stability with respect to local efficiency of at least 10, 20, or $30 \%$ respectively. For comparison, the expected welfare from a random network (based on 1000 random networks generated like in the simulations) and the maximal welfare have been calculated for each setting and are indicated in the table with $\mathrm{r}$ and $\mathrm{m}$ respectively.

\begin{tabular}{|c|l|l|l|l|l|l|l|l|l|}
\hline$\rho \backslash \alpha$ & \multicolumn{3}{|c|}{0} & \multicolumn{3}{c|}{$\frac{1}{2}$} & \multicolumn{3}{c|}{1} \\
\hline$\frac{1}{2}$ & 36.00 & 35.94 & $\mathrm{r} 33.3$ & 63.19 & 63.19 & $\mathrm{r} 50.2$ & 90.37 & 90.37 & $\mathrm{r} 67.0$ \\
& $(0.000)$ & $(0.087)$ & $\mathrm{m} 36.0$ & $(0.000)$ & $(0.000)$ & $\mathrm{m} 63.2$ & $(0.000)$ & $(0.000)$ & $\mathrm{m} 90.4$ \\
\hline$\frac{3}{4}$ & $17.81^{* * *}$ & 32.46 & $\mathrm{r} 20.1$ & $21.38^{* *}$ & 34.77 & $\mathrm{r} 26.0$ & $27.17^{* * *}$ & 38.97 & $\mathrm{r} 31.1$ \\
& $(3.26)$ & $(3.84)$ & $\mathrm{m} 36.0$ & $(3.43)$ & $(1.80)$ & $\mathrm{m} 36.0$ & $(4.44)$ & $(0.592)$ & $\mathrm{m} 39.2$ \\
\hline 1 & 29.16 & 30.00 & $\mathrm{r} 13.5$ & 31.86 & 30.36 & $\mathrm{r} 15.1$ & 32.88 & 32.21 & $\mathrm{r} 17.1$ \\
& $(8.15)$ & $(6.00)$ & $\mathrm{m} 36.0$ & $(4.49)$ & $(4.72)$ & $\mathrm{m} \mathrm{36.0}$ & $(3.08)$ & $(3.84)$ & $\mathrm{m} 36.0$ \\
\hline
\end{tabular}

Table 3.3: Welfare for Homogeneity 
Although we have seen that a higher $\alpha$ and thus more value transferability does not always lead to more dense networks, it apparently does always lead to higher welfare. Therefore, in a homogeneous setting, stimulating the focus on informational value in the community is a generally effective tool for boosting welfare. This effectivity is larger the smaller $\rho$. Furthermore, though a lower $\rho$ has been shown to lead to higher density, it only leads to subsequent higher welfare when the link specificity is low enough, since for intermediate values of $\rho$, welfare is much lower. This implies that enhancing communication in the community by decreasing link specificity from a high to an intermediate level counterintuitively results in lower welfare from communication.

We find that the structure difference found for $\alpha=0, \rho=\frac{1}{2}$ does not lead to a subsequent welfare difference, but for the intermediate level of specificity $\left(\rho=\frac{3}{4}\right)$, the tension between local efficiency and pairwise stability is considerable (even larger than $30 \%$ ).

\subsubsection{Homogeneity with Expert}

For homogeneity with expert as defined in (3.3), structure and payoff properties of the simulated pairwise stable and locally efficient networks are discussed. Before that, more general analytical results are provided for $\rho=1$ in line with Chapter 2.

\section{Analytical Results}

For $\rho=1$ and $0<\alpha<1$, if we assume a valuation pattern deviating from full homogeneity in the sense that there is one agent $j$ who is valued differently than all other agents, it can be proven that all pairwise stable structures consist of small star components and one possibly larger component without cycles containing the differing agent $j$ but not at the periphery. In particular, this component may be a star component with agent $j$ at the center. 
Proposition 25 Let $j \in N$, where $V_{i, j}^{\mathrm{i}}=V_{j}^{\mathrm{i}} \wedge V_{i, j}^{\mathrm{s}}=V_{j}^{\mathrm{s}} \forall i \neq j, V_{k, i}^{\mathrm{i}}=$ $V^{\mathrm{i}} \wedge V_{k, i}^{\mathrm{s}}=V^{\mathrm{s}} \forall i \neq j, \forall k \neq i$, and $\alpha V_{j}^{\mathrm{i}}+(1-\alpha) V_{j}^{\mathrm{s}} \neq \alpha V^{\mathrm{i}}+(1-\alpha) V^{\mathrm{s}}$. $A$ structure is pairwise stable if it consists of disjoint star components of two or three agents and a special star component with $j$ as the center agent, where the number of periphery agents $x \in\{1,2, \ldots, n-1\}$ satisfies

$$
x=1 \vee \alpha V_{j}^{\mathrm{i}} 3+(1-\alpha) V_{j}^{\mathrm{s}} 4 \geq \alpha V^{\mathrm{i}}(2 x-3)+(1-\alpha) V^{\mathrm{s}} 2 x,
$$

and if there is a three-agent star besides the special component also

$$
\begin{aligned}
\alpha V_{j}^{\mathrm{i}}+(1-\alpha) V_{j}^{\mathrm{s}} & >\alpha V^{\mathrm{i}} \frac{11 x^{2}-16 x+16}{16 x}+(1-\alpha) V^{\mathrm{s}} \frac{1}{2} x \\
\vee V_{j}^{\mathrm{i}} & <V^{\mathrm{i}} \frac{x^{2}-x+1}{x} \\
\vee \alpha V^{\mathrm{i}} \frac{5}{16} x & =(1-\alpha)\left[V^{\mathrm{s}} \frac{1}{2} x-V_{j}^{\mathrm{s}}\right] .
\end{aligned}
$$

Proof. It is not beneficial for any of the periphery agents in a star component to delete her single link as then her payoff will be zero. Equivalently, for the center agent in a star component of at least three agents, deleting a link with any of the periphery agents is not beneficial as it will provide her with the same payoff.

With respect to link creating possibilities, we have to examine the following cases $(a)-(n)$ :

\begin{tabular}{cl|c|c|c|c|c} 
& & 2 & $3 \mathrm{c}$ & $3 \mathrm{p}$ & $\mathrm{sc}$ & $\mathrm{sp}$ \\
\hline 2 & (pair agent) & $(a)$ & $(b)$ & $(c)$ & $(d)$ & $(e)$ \\
\hline $3 \mathrm{c}$ & (center agent of 3-agent star) & $\mathrm{x}$ & $(f)$ & $(g)$ & $(h)$ & $(i)$ \\
\hline $3 \mathrm{p}$ & (periphery agent of 3-agent star) & $\mathrm{x}$ & $\mathrm{x}$ & $(j),(k)^{2}$ & $(l)$ & $(m)$ \\
\hline $\mathrm{sc}$ & (center agent of special star $(j))$ & $\mathrm{x}$ & $\mathrm{x}$ & $\mathrm{x}$ & $\mathrm{x}$ & $\mathrm{x}$ \\
\hline $\mathrm{sp}$ & (periphery agent of special star) & $\mathrm{x}$ & $\mathrm{x}$ & $\mathrm{x}$ & $\mathrm{x}$ & $(n)$
\end{tabular}

\footnotetext{
${ }^{2}(j)$ is the case with two agents from different stars and $(k)$ is the case with two agents from the same star.
} 
For each of these cases, it can be proven by evaluating the payoffs with and without the link that no link is created. Cases $(a),(b),(c),(f),(g)$, $(j)$, and $(k)$ have been proven at Proposition 15 in Chapter 2.

In case $(d)$, the center agent of the special component would get payoff

$$
\begin{aligned}
& \alpha V^{\mathrm{i}}\left(\frac{1}{2(x+1)}+\frac{x}{x+1}+\frac{1}{4(x+1)}\right) \\
+ & (1-\alpha) V^{\mathrm{s}}\left(\frac{1}{2(x+1)}+\frac{x}{x+1}\right) \\
< & \alpha V^{\mathrm{i}}\left(\frac{x}{x}\right)+(1-\alpha) V^{\mathrm{s}}\left(\frac{x}{x}\right) .
\end{aligned}
$$

In case $(e)$, if the periphery agent of the special component would be willing to create the link, it should hold that

$$
\begin{aligned}
& \alpha\left[V^{\mathrm{i}}\left(\frac{1}{4}+\frac{1}{8}+\frac{x-1}{2 x^{2}}\right)+V_{j}^{\mathrm{i}} \frac{1}{2 x}\right]+(1-\alpha)\left[V^{\mathrm{s}} \frac{1}{4}+V_{j}^{\mathrm{s}} \frac{1}{2 x}\right] \\
\geq & \alpha\left[V^{\mathrm{i}} \frac{x-1}{x^{2}}+V_{j}^{\mathrm{i}} \frac{1}{x}\right]+(1-\alpha) V_{j}^{\mathrm{s}} \frac{1}{x},
\end{aligned}
$$

but then it follows that the payoff of the pair agent

$$
\begin{aligned}
& \alpha\left[V^{\mathrm{i}}\left(\frac{1}{2}+\frac{1}{4}+\frac{x-1}{8 x^{2}}\right)+V_{j}^{\mathrm{i}} \frac{1}{8 x}\right] \\
& +(1-\alpha) V^{\mathrm{s}}\left(\frac{1}{2}+\frac{1}{4}\right)
\end{aligned}
$$

would be smaller than $\alpha V^{\mathrm{i}}+(1-\alpha) V^{\mathrm{s}}$.

In case $(h)$, the center agent of the special component would get payoff

$$
\begin{aligned}
& \alpha V^{\mathrm{i}}\left(\frac{1}{3(x+1)}+\frac{x}{x+1}+\frac{2}{9(x+1)}\right) \\
+ & (1-\alpha) V^{\mathrm{s}}\left(\frac{1}{3(x+1)}+\frac{x}{x+1}\right) \\
< & \alpha V^{\mathrm{i}}\left(\frac{x}{x}\right)+(1-\alpha) V^{\mathrm{s}}\left(\frac{x}{x}\right) .
\end{aligned}
$$


In case $(i)$, if the periphery agent of the special component would be willing to create the link, it should hold that

$$
\begin{aligned}
& \alpha\left[V^{\mathrm{i}}\left(\frac{1}{6}+\frac{2}{18}+\frac{x-1}{2 x^{2}}\right)+V_{j}^{\mathrm{i}} \frac{1}{2 x}\right]+(1-\alpha)\left[V^{\mathrm{s}} \frac{1}{6}+V_{j}^{\mathrm{s}} \frac{1}{2 x}\right] \\
\geq & \alpha\left[V^{\mathrm{i}} \frac{x-1}{x^{2}}+V_{j}^{\mathrm{i}} \frac{1}{x}\right]+(1-\alpha) V_{j}^{\mathrm{s}} \frac{1}{x},
\end{aligned}
$$

but then it follows that the payoff of the center agent of the three-agent star

$$
\begin{aligned}
& \alpha\left[V^{\mathrm{i}}\left(\frac{2}{3}+\frac{1}{6}+\frac{x-1}{12 x^{2}}\right)+V_{j}^{\mathrm{i}} \frac{1}{12 x}\right] \\
& +(1-\alpha) V^{\mathrm{s}}\left(\frac{2}{3}+\frac{1}{6}\right)
\end{aligned}
$$

would be smaller than $\alpha V^{\mathrm{i}}+(1-\alpha) V^{\mathrm{s}}$.

In case $(l)$, the center agent of the special component would get payoff

$$
\begin{aligned}
& \quad \alpha V^{\mathrm{i}}\left(\frac{1}{2(x+1)}+\frac{x}{x+1}+\frac{1}{8(x+1)}+\frac{1}{16(x+1)}\right) \\
& +(1-\alpha) V^{\mathrm{s}}\left(\frac{1}{2(x+1)}+\frac{x}{x+1}\right) \\
& <\alpha V^{\mathrm{i}}\left(\frac{x}{x}\right)+(1-\alpha) V^{\mathrm{s}}\left(\frac{x}{x}\right) .
\end{aligned}
$$

In case $(m)$, either the periphery agent of the special component would get payoff

$$
\alpha\left[V^{\mathrm{i}}\left(\frac{1}{4}+\frac{1}{16}+\frac{1}{32}+\frac{x-1}{2 x^{2}}\right)+V_{j}^{\mathrm{i}} \frac{1}{2 x}\right]+(1-\alpha)\left[V^{\mathrm{s}} \frac{1}{4}+V_{j}^{\mathrm{s}} \frac{1}{2 x}\right],
$$

which is smaller than

$$
\alpha\left[V^{\mathrm{i}} \frac{x-1}{x^{2}}+V_{j}^{\mathrm{i}} \frac{1}{x}\right]+(1-\alpha) V_{j}^{\mathrm{s}} \frac{1}{x}
$$


because of condition (3.7), or the periphery agent of the three-agent star would get payoff

$$
\alpha\left[V^{\mathrm{i}}\left(\frac{2}{4}+\frac{1}{8}+\frac{x-1}{8 x^{2}}\right)+V_{j}^{\mathrm{i}} \frac{1}{8 x}\right]+(1-\alpha) V^{\mathrm{s}} \frac{2}{4},
$$

which is smaller than

$$
\alpha V^{\mathrm{i}}\left(\frac{1}{2}+\frac{1}{4}\right)+(1-\alpha) V^{\mathrm{s}} \frac{1}{2}
$$

because of condition (3.8), or both agents would be equally off because of condition (3.9).

In case $(n)$, where $x \geq 2$, each agent would get payoff

$$
\begin{aligned}
& \alpha\left[V^{\mathrm{i}}\left(\frac{1}{4}+\frac{1}{4 x^{2}}+\frac{x-2}{2 x^{2}}+\frac{x-2}{8 x^{2}}\right)+V_{j}^{\mathrm{i}}\left(\frac{1}{2 x}+\frac{1}{8 x}\right)\right] \\
& +(1-\alpha)\left[V^{\mathrm{s}} \frac{1}{4}+V_{j}^{\mathrm{s}} \frac{1}{2 x}\right],
\end{aligned}
$$

which is not larger than

$$
\alpha\left[V^{\mathrm{i}} \frac{x-1}{x^{2}}+V_{j}^{\mathrm{i}} \frac{1}{x}\right]+(1-\alpha) V_{j}^{\mathrm{s}} \frac{1}{x}
$$

because of condition (3.6).

Proposition 26 Let $j \in N$, where $V_{i, j}^{\mathrm{i}}=V_{j}^{\mathrm{i}} \wedge V_{i, j}^{\mathrm{s}}=V_{j}^{\mathrm{s}} \forall i \neq j, V_{k, i}^{\mathrm{i}}=$ $V^{\mathrm{i}} \wedge V_{k, i}^{\mathrm{s}}=V^{\mathrm{s}} \forall i \neq j, \forall k \neq i$, and $\alpha V_{j}^{\mathrm{i}}+(1-\alpha) V_{j}^{\mathrm{s}} \neq \alpha V^{\mathrm{i}}+(1-\alpha) V^{\mathrm{s}} . A$ structure is only pairwise stable if it consists of disjoint star components of two or three agents and a special component without cycles containing agent $j$, who is not located at the periphery if there is at least one non-periphery agent.

Proof. The proof that the pairwise stable structure $g$ does not contain cycles is the same as with Proposition 15 in Chapter 2. Also it follows that when a component of $g$ does not contain agent $j$ and therefore only 
consists of homogenous agents, it must be a star of two or three agents. A tree component with $j$ in the periphery cannot be part of $g$, since it is always strictly beneficial for some non-periphery agent of such a component to delete the link with her neighbor either at the side of $j$ or at the other side, for $\alpha V_{j}^{i}+(1-\alpha) V_{j}^{s} \neq \alpha V^{i}+(1-\alpha) V^{s}$.

The following example illustrates that the conditions given in Proposition 25 are not necessary for pairwise stability.

Example 27 Assume $V_{j}^{\mathrm{i}}=V_{j}^{\mathrm{s}}=\frac{29}{16}, V^{\mathrm{i}}=V^{\mathrm{s}}=1, \alpha=\frac{1}{2}$. It is easy to see that for these particular valuations, the following structure is pairwise stable.

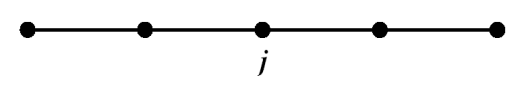

Figure 3.1: A Non-Star Pairwise Stable Structure in the Expert Case

\section{Structure Properties}

With the specific expert valuation pattern defined for our simulations in (3.3), condition (3.6) reduces to

$$
x=1 \vee x \leq 14-2 \alpha,
$$

which holds for any possible $x$ given $n=6$. Also, condition (3.7) reduces to

$$
(3 \alpha+8) x^{2}-(16 \alpha+112) x+16 \alpha<0
$$

and thus there is no further restriction on $x$ for the case that there is a three-agent star besides the special component. This result is confirmed by the simulation outcomes: for $\rho=1$ and $\alpha=\frac{1}{2}$, each pairwise stable network consists of two- and three-agent stars and in 36 cases a star component with agent 1 in the centre and three or five periphery agents. 
For each parameter setting, the average density and the standard deviation of the simulated pairwise stable and locally efficient networks are given in Table 3.4.

\begin{tabular}{|c|l|l|l|l|l|l|}
\hline$\rho \backslash \alpha$ & \multicolumn{2}{|c|}{0} & \multicolumn{2}{c|}{$\frac{1}{2}$} & \multicolumn{2}{c|}{1} \\
\hline$\frac{1}{2}$ & 0.333 & 0.333 & $0.976^{* *}$ & 0.721 & 1.00 & 1.00 \\
& $(0.000)$ & $(0.000)$ & $(0.076)$ & $(0.026)$ & $(0.000)$ & $(0.000)$ \\
\hline$\frac{3}{4}$ & 0.319 & 0.246 & $0.333^{*}$ & 0.229 & 0.333 & 0.333 \\
& $(0.061)$ & $(0.031)$ & $(0.000)$ & $(0.033)$ & $(0.000)$ & $(0.000)$ \\
\hline 1 & 0.263 & 0.219 & 0.245 & 0.234 & 0.253 & 0.233 \\
& $(0.047)$ & $(0.030)$ & $(0.038)$ & $(0.033)$ & $(0.044)$ & $(0.033)$ \\
\hline
\end{tabular}

Table 3.4: Densities for Homogeneity with Expert

Although some density values thus are lower than in the completely homogeneous case (Table 3.1), the basic intuition that increasing $\rho$ implies decreasing density is again confirmed by the simulation outcomes. Also, the intuition about the positive effect of $\alpha$ on density is realized by the simulation outcomes more than with complete homogeneity.

Since the densities of the locally efficient and pairwise stable structures do not differ that much, we also look at the relative centralities. For each parameter setting, the average relative centrality and the standard deviation of all agents in the simulated pairwise stable and locally efficient networks are given in Table 3.5 .

Apparently, decreasing $\rho$ leads to more centrality for the expert first, after which the density increases further such that the centrality of the expert decreases again. Also $\alpha$ does not have a consistent effect on expert centrality.

For this valuation pattern we do find considerable differences between pairwise stable and locally efficient structures: except for $\rho=\frac{1}{2}$, the relative centrality of the expert is higher in the pairwise stable networks than in the locally efficient ones. In the following example we illustrate the case with the largest centrality difference.

Example 28 For $\rho=\frac{3}{4}$ and $\alpha=\frac{1}{2}$, Table 3.6 provides an overview of the types and numbers of pairwise stable networks resulting from 100 simulations 


\begin{tabular}{|c|c|c|c|c|c|c|}
\hline$\rho \backslash \alpha$ & \multicolumn{2}{|c|}{0} & \multicolumn{2}{|c|}{$\overline{5}$} & \multicolumn{2}{|c|}{1} \\
\hline \multirow{12}{*}{$\frac{1}{2}$} & 1.00 & 1.00 & $0.016^{*}$ & 0.194 & 0.000 & 0.000 \\
\hline & $(0.000)$ & $(0.000)$ & $(0.052)$ & $(0.026)$ & $(0.000)$ & $(0.000)$ \\
\hline & 2. -0.200 & ๑. -0.200 & .. 0.004 & -0.036 & 0.000 & 0.000 \\
\hline & 2: $\quad(0.000)$ & 2: (0.000) & 2: $\quad(0.013)$ & 2: (0.061) & 2: $\quad(0.000)$ & 2: (0.000) \\
\hline & -0.200 & -0.200 & 0.000 & -0.037 & 0.000 & 0.000 \\
\hline & $3: \quad(0.000)$ & 3: $(0.000)$ & $3: \quad(0.039)$ & $(0.061)$ & $(0.000)$ & $(0.000)$ \\
\hline & -0.200 & -0.200 & -0.008 & -0.037 & 0.000 & 0.000 \\
\hline & 4: $\quad(0.000)$ & 4: $\quad(0.000)$ & 4: $\quad(0.063)$ & 4: $\quad(0.061)$ & 4: $\quad(0.000)$ & $(0.000)$ \\
\hline & -0.200 & -0.200 & 5. 0.000 & -0.037 & 0.000 & 0.000 \\
\hline & $5: \quad(0.000)$ & 3: $\quad(0.000)$ & $(0.039)$ & $(0.061)$ & $(0.000)$ & $(0.000)$ \\
\hline & 6. -0.200 & 6. -0.200 & 6. -0.012 & 6. -0.047 & 6. 0.000 & 0.000 \\
\hline & 6: $\quad(0.000)$ & b: $\quad(0.000)$ & $(0.072)$ & $(0.058)$ & $(0.000)$ & $(0.000)$ \\
\hline \multirow{12}{*}{$\frac{3}{4}$} & 1. $0.497^{* *}$ & 1. 0.259 & 1. $0.925^{* *}$ & 0.189 & $1.00^{* * *}$ & 0.418 \\
\hline & $1: \quad(0.418)$ & 1: (0.239) & 1: $\quad(0.276)$ & 1: $\quad(0.247)$ & $(0.000)$ & $(0.083)$ \\
\hline & 2. -0.109 & ๑. -0.056 & 2. $-0.185^{*}$ & ๑. -0.040 & 2. $-0.200^{*}$ & ๑. -0.059 \\
\hline & $2: \quad(0.118)$ & $\left(0.10^{r}\right)$ & $2: \quad(0.065)$ & $(0.085)$ & $2: \quad(0.000)$ & $(0.150)$ \\
\hline & -0.098 & -0.049 & 2. $-0.185^{*}$ & ๑. -0.025 & 2. $-0.200^{*}$ & -0.095 \\
\hline & $3: \quad(0.122)$ & $3: \quad(0.126)$ & $3: \quad(0.065)$ & $(0.100)$ & $3: \quad(0.000)$ & $(0.143)$ \\
\hline & -0.111 & -0.041 & 4. $-0.185^{*}$ & 1. -0.040 & 4. $-0.200^{*}$ & -0.080 \\
\hline & 4: $\quad(0.119)$ & 4: (0.132) & 4: $\quad(0.065)$ & 4: $\quad(0.085)$ & 4: $\quad(0.000)$ & 4: $(0.147)$ \\
\hline & 5. -0.093 & 5. -0.056 & 5. $-0.191^{*}$ & -0.044 & 5. $-0.200^{*}$ & -0.098 \\
\hline & S: $\quad(0.125)$ & 5: $\quad(0.107)$ & $(0.051)$ & $(0.080)$ & $(0.000)$ & $(0.142)$ \\
\hline & 6. -0.086 & 6. -0.056 & $-0.179^{*}$ & -0.040 & $-0.200^{*}$ & -0.086 \\
\hline & 6: $\quad(0.126)$ & b: $\quad(0.107)$ & $6: \quad(0.077)$ & $(0.085)$ & 6: $\quad(0.000)$ & $(0.146)$ \\
\hline \multirow{12}{*}{1} & $0.366^{* *}$ & 0.111 & 1. $0.311^{*}$ & 0.113 & 1. $0.308^{*}$ & 0.129 \\
\hline & 1: $\quad(0.369)$ & I: (0.223) & $1: \quad(0.302)$ & (0.232) & 1: $\quad(0.361)$ & 1: (0.227) \\
\hline & -0.072 & ๑. -0.016 & -0.053 & -0.030 & -0.071 & -0.033 \\
\hline & $\begin{array}{l}2: \quad(0.107) \\
0\end{array}$ & (0.156) & L: $\quad(0.110)$ & $(0.130)$ & 2: $\quad(0.108)$ & $(0.127)$ \\
\hline & 2. -0.085 & ฉ. -0.006 & -0.071 & -0.011 & -0.067 & -0.014 \\
\hline & $3 . \quad(0.095)$ & $3: \quad(0.140)$ & 3. $\quad(0.086)$ & $(0.160)$ & $(0.124)$ & $(0.139)$ \\
\hline & -0.079 & -0.014 & -0.064 & -0.019 & -0.048 & -0.018 \\
\hline & 4: $\quad(0.101)$ & 4: (0.125) & 4: $\quad(0.097)$ & $(0.165)$ & $(0.151)$ & $(0.147)$ \\
\hline & 5. -0.069 & -0.034 & -0.071 & -0.030 & -0.052 & -0.036 \\
\hline & 5: $\quad(0.107)$ & 3: (0.112) & $(0.086)$ & $(0.130)$ & $(0.157)$ & $(0.124)$ \\
\hline & $6:-0.061$ & $6:-0.041$ & $6:-0.053$ & -0.023 & -0.071 & $6:-0.029$ \\
\hline & & (0.090) & $(0.110)$ & $(0.154)$ & $(0.120)$ & $(0.140)$ \\
\hline
\end{tabular}

Table 3.5: Centralities for Homogeneity with Expert

and the types and numbers of locally efficient networks resulting from another 100 simulations.

Thus, in the pairwise stable networks the expert takes a central position as with lower levels of link specificity and value transferability, whereas in the locally efficient networks the expert is only slightly more central than the other community members as with higher levels of link specificity and value transferability. 


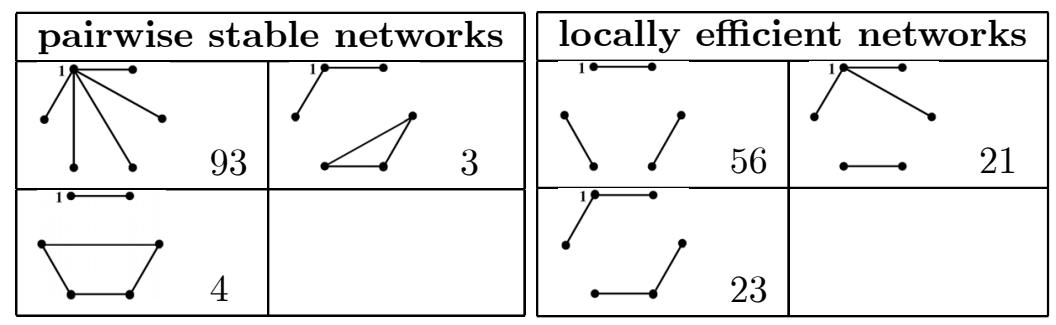

Table 3.6: Example of Structures for Homogeneity with Expert

\section{Payoff Properties}

As a means to investigate in how far the structure differences between the locally efficient and pairwise stable networks actually lead to welfare differences, for each parameter setting, the average welfare and the standard deviation of the simulated pairwise stable and locally efficient networks are given in Table 3.7 .

\begin{tabular}{|c|c|c|c|c|c|c|c|c|c|}
\hline$\rho \backslash \alpha$ & \multicolumn{3}{|c|}{0} & \multicolumn{3}{|c|}{$\frac{1}{2}$} & \multicolumn{3}{|c|}{1} \\
\hline$\frac{1}{2}$ & $\begin{array}{l}53.67 \\
(0.000)\end{array}$ & $\begin{array}{l}53.67 \\
(0.000)\end{array}$ & $\begin{array}{l}\mathrm{r} 33.2 \\
\mathrm{~m} 53.7\end{array}$ & $\begin{array}{l}63.07 \\
(0.370)\end{array}$ & $\begin{array}{l}64.04 \\
(0.110)\end{array}$ & $\begin{array}{l}\mathrm{r} 50.3 \\
\mathrm{~m} 64.1\end{array}$ & $\begin{array}{l}90.37 \\
(0.000)\end{array}$ & $\begin{array}{l}90.37 \\
(0.000)\end{array}$ & $\begin{array}{l}\mathrm{r} 67.5 \\
\mathrm{~m} 90.4\end{array}$ \\
\hline$\frac{3}{4}$ & $\begin{array}{l}35.82 \\
(2.02)\end{array}$ & $\begin{array}{l}36.18 \\
(0.989)\end{array}$ & $\begin{array}{l}\mathrm{r} 21.0 \\
\mathrm{~m} 37.6\end{array}$ & $\begin{array}{l}38.36 \\
(0.825)\end{array}$ & $\begin{array}{l}37.11 \\
(1.35)\end{array}$ & $\begin{array}{l}\mathrm{r} 26.2 \\
\mathrm{~m} 39.3\end{array}$ & $\begin{array}{l}41.25 \\
(0.000)\end{array}$ & $\begin{array}{l}41.02 \\
(0.149)\end{array}$ & $\begin{array}{l}\mathrm{r} 31.1 \\
\mathrm{~m} 41.5\end{array}$ \\
\hline 1 & $\begin{array}{l}30.81 \\
(3.71)\end{array}$ & $\begin{array}{l}33.78 \\
(2.90)\end{array}$ & $\begin{array}{l}\text { r } 13.6 \\
\text { m } 36.0\end{array}$ & $\begin{array}{l}32.70 \\
(2.85)\end{array}$ & $\begin{array}{l}33.56 \\
(2.40)\end{array}$ & $\begin{array}{l}\mathrm{r} 15.4 \\
\mathrm{~m} 36.0\end{array}$ & $\begin{array}{l}32.86 \\
(3.06)\end{array}$ & $\begin{array}{l}34.25 \\
(1.79)\end{array}$ & $\begin{array}{l}\mathrm{r} 16.8 \\
\mathrm{~m} 36.0\end{array}$ \\
\hline
\end{tabular}

Table 3.7: Welfare for Homogeneity with Expert

Again we find that a higher $\alpha$ and thus more value transferability always leads to higher welfare. Therefore, also in a homogeneous setting with one expert, stimulating the focus on informational value in the community is a generally effective tool for boosting welfare. Moreover, in this setting a lower $\rho$ also consistently leads to subsequent higher welfare. This implies that enhancing communication in the community by decreasing link specificity becomes a complementary tool to increase welfare from communication.

Apparently, the difference in centralization does not lead to any serious (> 10\%) tension between overall local efficiency and pairwise stability. Also on the individual payoff level there are no large payoff differences between pairwise stable and locally efficient networks, nor for the expert nor for the 
other community members. Comparing Tables 3.3 and 3.7, adding an expert to a homogeneous community appears to be an effective welfare-increasing tool for intermediate link specificity.

\subsubsection{Expertise Heterogeneity}

For expertise heterogeneity as defined in (3.4), structure and payoff properties of the simulated pairwise stable and locally efficient networks are discussed. Before that, a general analytical result is provided for $\rho=1$ in line with Chapter 2.

\section{Analytical Result}

For $\rho=1$ and $0<\alpha<1$, if we assume any heterogeneous valuation pattern, the fragmented pairwise stable structure of the more homogeneous cases persists, where highly valued agents tend to link to each other. It can be proven that there always exists a pair structure that is pairwise stable, which can be found by ranking agents with respect to their valuation.

Proposition 29 If $n$ is even and if the agents are ranked such that it holds for agent $i(r)$ with rank $r=1,2, \ldots, n$ that

$$
\alpha V_{i(r)}^{\mathrm{i}}+(1-\alpha) V_{i(r)}^{\mathrm{s}} \geq \alpha V_{i(r+1)}^{\mathrm{i}}+(1-\alpha) V_{i(r+1)}^{\mathrm{s}}
$$

the pairwise structure in which $i(r)$ is linked with $i(r+1)$, where $r=$ $1,3,5, \ldots, n-1$, is pairwise stable.

Proof. It is never beneficial for any of the agents to delete her link, as then her payoff will be zero. Moreover, no link is created if any agent strictly looses by linking to a lower-ranked agent - i.e., an agent with higher $r$ - from 
another pair, so if $\forall r \in\{1,3,5, \ldots, n-1\} \wedge \forall x \in\{2,4,6, \ldots, n-r-1\}$ :

$$
\begin{aligned}
\alpha V_{i(r)}^{\mathrm{i}}+(1-\alpha) V_{i(r)}^{\mathrm{s}}> & \alpha\left(V_{i(r)}^{\mathrm{i}} \frac{1}{2}+V_{i(r+x)}^{\mathrm{i}} \frac{1}{4}+V_{i(r+x+1)}^{\mathrm{i}} \frac{1}{8}\right) \\
& +(1-\alpha)\left(V_{i(r) \frac{1}{2}}^{\mathrm{s}}+V_{i(r+x)}^{\mathrm{s}} \frac{1}{4}\right) \\
\wedge \alpha V_{i(r)}^{\mathrm{i}}+(1-\alpha) V_{i(r)}^{\mathrm{s}}> & \alpha\left(V_{i(r)}^{\mathrm{i}} \frac{1}{2}+V_{i(r+x+1)}^{\mathrm{i}}+V_{i(r+x)}^{\mathrm{i}} \frac{1}{4}\right) \\
& +(1-\alpha)\left(V_{\left.i(r) \frac{1}{2}+V_{i(r+x+1)}^{\mathrm{s}} \frac{1}{4}\right)}^{\mathrm{s}}(3.12)\right. \\
\wedge \alpha V_{i(r+1)}^{\mathrm{i}}+(1-\alpha) V_{i(r+1)}^{\mathrm{s}}> & \alpha\left(V_{i(r+1)}^{\mathrm{i}} \frac{1}{2}+V_{i(r+x)}^{\mathrm{i}} \frac{1}{4}+V_{i(r+x+1)}^{\mathrm{i}} \frac{1}{8}\right) \\
& +(1-\alpha)\left(V_{i(r+1)}^{\mathrm{s}} \frac{1}{2}+V_{i(r+x)}^{\mathrm{s}} \frac{1}{4}\right) \quad(3.13) \\
\wedge \alpha V_{i(r+1)}^{\mathrm{i}}+(1-\alpha) V_{i(r+1)}^{\mathrm{s}}> & \alpha\left(V_{i(r+1)}^{\mathrm{i}} \frac{1}{2}+V_{i(r+x+1)}^{\mathrm{i}}+V_{i(r+x)}^{\mathrm{i}} \frac{1}{8}\right) \\
& +(1-\alpha)\left(V_{i(r+1)}^{\mathrm{s}}+V_{i(r+x+1)}^{\mathrm{s}} \frac{1}{4}\right)(3.14)
\end{aligned}
$$

We show (3.11), whereas (3.12), (3.13), and (3.14) are similar. It follows from (3.10) that

$$
\frac{1}{4}\left(\alpha V_{i(r)}^{\mathrm{i}}+(1-\alpha) V_{i(r)}^{\mathrm{s}}\right) \geq \frac{1}{4}\left(\alpha V_{i(r+x)}^{\mathrm{i}}+(1-\alpha) V_{i(r+x)}^{\mathrm{s}}\right)
$$

and that

$$
\frac{1}{8}\left(\alpha V_{i(r)}^{\mathrm{i}}+(1-\alpha) V_{i(r)}^{\mathrm{s}}\right)>\frac{1}{8} \alpha V_{i(r+x+1)}^{\mathrm{i}} .
$$

Therefore, it holds that

$$
\frac{3}{8}\left(\alpha V_{i(r)}^{\mathrm{i}}+(1-\alpha) V_{i(r)}^{\mathrm{s}}\right)>\alpha\left(V_{i(r+x)}^{\mathrm{i}} \frac{1}{4}+V_{i(r+x+1)}^{\mathrm{i}} \frac{1}{8}\right)+(1-\alpha) V_{i(r+x)}^{\mathrm{s}} \frac{1}{4},
$$

from which (3.11) follows.

This type of ranking by agents is even more thinkable since rankings by fellow consumers are publicly recorded in many OCCNs, see for example on forums.itrc.hp.com/cm/. 
Note that the network structures that only consists of pairs, which are formed according to the ranking system, need not be the only pairwise stable structures. In particular, like in the more homogeneous cases, all kinds of structures consisting of small, disjoint star components, can be pairwise stable. As suggested by Section 3.5.3, the smallness of these star components can be partly eliminated by the presence of an expert in the field, but is stimulated again by heterogeneity among consumers, which causes experts to form small components with each other.

\section{Structure Properties}

With the specific heterogeneous valuation pattern defined for our simulations in (3.4), $\rho=1$ and $\alpha=\frac{1}{2}$, inequality (3.10) is fulfilled when $r=i \forall r$. This result is confirmed by the simulation outcomes: 42 of the 100 simulated pairwise stable networks consist of the disjoint pair components 1-2, 3-4, and 5-6. Moreover, all other pairwise stable networks also only consist of pairs that do not link the extremely valuated agents to each other: 1-3, 2-4, 5-6 (22); 1-4, 2-3, 5-6 (21); 1-2, 3-5, 4-6 (12); and 1-3, 2-5, 4-6 (3).

For each parameter setting, the average density and the standard deviation of the simulated pairwise stable and locally efficient networks are given in Table 3.8.

\begin{tabular}{|c|l|l|l|l|l|l|}
\hline$\rho \backslash \alpha$ & \multicolumn{2}{|c|}{0} & \multicolumn{2}{|c|}{$\frac{1}{2}$} & \multicolumn{2}{c|}{1} \\
\hline$\frac{1}{2}$ & 0.533 & 0.461 & $0.789^{*}$ & 0.927 & 0.977 & 1.00 \\
& $(0.000)$ & $(0.107)$ & $(0.133)$ & $(0.019)$ & $(0.101)$ & $(0.000)$ \\
\hline$\frac{3}{4}$ & 0.301 & 0.215 & 0.265 & 0.231 & $0.236^{*}$ & 0.343 \\
& $(0.082)$ & $(0.028)$ & $(0.067)$ & $(0.033)$ & $(0.059)$ & $(0.023)$ \\
\hline 1 & 0.200 & 0.200 & 0.200 & 0.203 & 0.200 & 0.212 \\
& $(0.000)$ & $(0.000)$ & $(0.000)$ & $(0.013)$ & $(0.000)$ & $(0.026)$ \\
\hline
\end{tabular}

Table 3.8: Densities for Expertise Heterogeneity

The density values thus are more similar to the expert case (Table 3.4) than to the completely homogeneous case (Table 3.1); the basic intuition that increasing $\rho$ implies decreasing density is again confirmed by the simulation outcomes and once more the intuition about the positive effect of $\alpha$ on density is not always realized by the simulation outcomes. 
Since again the densities of the locally efficient and pairwise stable structures do not differ that much, for each parameter setting, the average relative centrality and the standard deviation of all agents in the simulated pairwise stable and locally efficient networks are given in Table 3.9.

\begin{tabular}{|c|c|c|c|c|c|c|}
\hline$\rho \backslash \alpha$ & \multicolumn{2}{|c|}{0} & \multicolumn{2}{|c|}{$\frac{1}{2}$} & \multicolumn{2}{|c|}{1} \\
\hline \multirow{12}{*}{$\frac{1}{2}$} & 1. 0.250 & 1. 0.340 & 1. 0.070 & 0.039 & 1. 0.006 & 0.000 \\
\hline & 1. $\quad(0.000)$ & 1. (0.092) & 1. $\quad(0.032)$ & 1. (0.012) & $(0.028)$ & 1. $(0.000)$ \\
\hline & 0.179 & 0.152 & 0.070 & 0.039 & 0.006 & 0.000 \\
\hline & 2: $\quad(0.091)$ & 2: $(0.084)$ & 2: $\quad(0.032)$ & (0.012) & $(0.028)$ & $(0.000)$ \\
\hline & 3. $0.134^{*}$ & 0.018 & 3. 0.070 & 3. 0.029 & 3. 0.006 & 0.000 \\
\hline & $3: \quad(0.091)$ & $(0.081)$ & $3: \quad(0.032)$ & (0.021) & $(0.028)$ & $(0.000)$ \\
\hline & 4. $\quad 0.063$ & -0.070 & 0.070 & 4. 0.029 & 0.006 & 0.000 \\
\hline & 4: $\quad(0.000)$ & 4: $\quad(0.073)$ & 4: $\quad(0.032)$ & 4: $\quad(0.021)$ & $(0.028)$ & $(0.000)$ \\
\hline & 5. -0.125 & 5. -0.172 & 5. 0.021 & 5. -0.068 & -0.007 & 0.000 \\
\hline & 5: $\quad(0.000)$ & 3: $\quad(0.067)$ & 5: $\quad(0.085)$ & 3: (0.009) & $(0.051)$ & $(0.000)$ \\
\hline & $6:-0.500^{*}$ *⿻丷木 & $6 \cdot-0.269$ & 6. $-0.303^{* *}$ & 6. $\quad-0.068$ & -0.019 & 0.000 \\
\hline & 0. $\quad(0.000)$ & $0 . \quad(0.064)$ & $(0.214)$ & $0 . \quad(0.009)$ & $(0.084)$ & $(0.000)$ \\
\hline \multirow{12}{*}{$\frac{3}{4}$} & 1. $0.359^{* *}$ & 1. 0.069 & 1. $0.336^{* *}$ & 1. 0.091 & $0.313^{*}$ & 0.122 \\
\hline & 1. $\quad(0.182)$ & 1. $(0.183)$ & 1. $\quad(0.188)$ & 1. (0.153) & 1. $(0.218)$ & $(0.108)$ \\
\hline & 2. $0.132^{*}$ & ๑. 0.020 & 2. 0.049 & ๑. 0.031 & 0.027 & 0.086 \\
\hline & 2: $\quad(0.109)$ & 2: (0.122) & $2: \quad(0.050)$ & 2: $\quad(0.161)$ & $(0.044)$ & $(0.035)$ \\
\hline & 0.008 & -0.010 & 0.049 & 0.028 & 0.027 & 0.083 \\
\hline & $3: \quad(0.066)$ & 3: $\quad(0.076)$ & $3: \quad(0.050)$ & $3: \quad(0.152)$ & $(0.044)$ & (0.062) \\
\hline & -0.064 & -0.021 & 4. -0.098 & 1. -0.033 & -0.024 & -0.031 \\
\hline & 4: $\quad(0.087)$ & 4: $(0.064)$ & 4: $\quad(0.100)$ & 4: $(0.098)$ & $(0.085)$ & $(0.139)$ \\
\hline & 5. $-0.139^{*}$ & 5. -0.029 & 5. -0.098 & 5. -0.059 & -0.054 & -0.091 \\
\hline & 5: $\quad(0.105)$ & $5: \quad(0.053)$ & $5: \quad(0.100)$ & 5: (0.062) & $(0.089)$ & $(0.134)$ \\
\hline & 6. $-0.294^{*}$ ** & 6. -0.029 & 6. $-0.238^{*}$ & -0.059 & $-0.289^{*}$ & -0.169 \\
\hline & o: $\quad(0.200)$ & $0: \quad(0.053)$ & o: $\quad(0.182)$ & $0: \quad(0.062)$ & $(0.212)$ & $(0.074)$ \\
\hline \multirow{12}{*}{1} & 1. 0.000 & 1. 0.000 & 1. 0.000 & 1. 0.029 & 0.000 & 0.021 \\
\hline & 1: $\quad(0.000)$ & 1. $(0.000)$ & 1. $\quad(0.000)$ & $1 . \quad(0.124)$ & $(0.000)$ & $(0.131)$ \\
\hline & 2. 0.000 & ๑. 0.000 & 2. 0.000 & ๑. 0.003 & 0.000 & -0.006 \\
\hline & 2: $\quad(0.000)$ & $2: \quad(0.000)$ & Z: $\quad(0.000)$ & 2: (0.039) & $(0.000)$ & (0.082) \\
\hline & 3. 0.000 & ง. 0.000 & 3. 0.000 & -0.005 & 0.000 & -0.015 \\
\hline & $3: \quad(0.000)$ & $3: \quad(0.000)$ & 3: $\quad(0.000)$ & $(0.024)$ & $(0.000)$ & $(0.059)$ \\
\hline & 4. 0.000 & 0.000 & 4. 0.000 & -0.005 & 0.000 & 0.008 \\
\hline & $4: \quad(0.000)$ & $4:(0.000)$ & $4: \quad(0.000)$ & $(0.024)$ & $(0.000)$ & $(0.010)$ \\
\hline & 5. 0.000 & 5. 0.000 & 5: $\quad 0.000$ & -0.001 & 0.000 & -0.004 \\
\hline & J. $(0.000)$ & . (0.000) & $(0.000)$ & $(0.033)$ & $(0.000)$ & $(0.089)$ \\
\hline & 6: $\quad \begin{array}{l}0.000 \\
(0.000)\end{array}$ & $6: \begin{array}{l}0.000 \\
0(00 \Omega)\end{array}$ & 6: $\quad \begin{array}{l}0.000 \\
(0000)\end{array}$ & $6: \quad-0.020$ & 6: $\stackrel{0.000}{(0000)}$ & -0.004 \\
\hline & $(0.000)$ & $(0.000)$ & & & & $(0.155)$ \\
\hline
\end{tabular}

Table 3.9: Centralities for Expertise Heterogeneity

Similarly to the expert case, decreasing $\rho$ leads to more centrality for the highest valued agent and furthermore less centrality for the lowest valued agent first, after which the density increases further such that these centralities become closer again. Also $\alpha$ does not always have a consistent effect on relative centralities. 


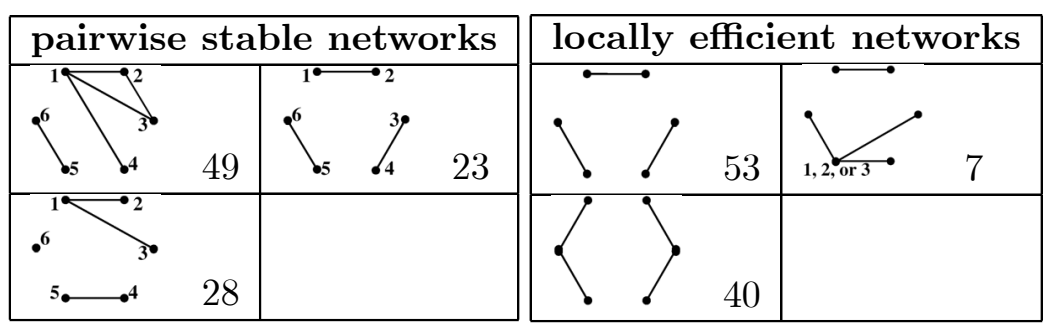

Table 3.10: Example of Structures for Expertise Heterogeneity

Now we do find differences between pairwise stable and locally efficient structures: except for $\rho=1$ with high fragmentation, the difference between the relative centralities of the most and least highly valued agents is higher in the pairwise stable networks than in the locally efficient ones. In the following example we illustrate one of these cases.

Example 30 Let $\rho=\frac{3}{4}, \alpha=\frac{1}{2}$, then Table 3.10 provides an overview of the types and numbers of pairwise stable networks resulting from 100 simulations and the types and numbers of locally efficient networks resulting from another 100 simulations.

Thus, in the pairwise stable networks agent 1 is relatively central and agent 6 is relatively isolated, whereas in the locally efficient networks all agents are more equally centralized.

\section{Payoff Properties}

As a means to investigate in how far the structure differences between the locally efficient and stable networks actually lead to welfare differences, for each parameter setting, the average welfare and the standard deviation of the simulated pairwise stable and locally efficient networks are given in Table 3.11 .

Except for $\rho=1$, we again find that a higher $\alpha$ and thus more value transferability leads to higher welfare. Therefore, also in a heterogeneous setting, stimulating the focus on informational value in the community is an effective tool for boosting welfare. However, in this setting a lower $\rho$ does 


\begin{tabular}{|c|c|c|c|c|c|c|c|c|c|}
\hline$\rho \backslash \alpha$ & \multicolumn{3}{|c|}{0} & \multicolumn{3}{|c|}{$\frac{1}{2}$} & \multicolumn{3}{|c|}{1} \\
\hline$\frac{1}{2}$ & $\begin{array}{l}36.46 \\
(0.262)\end{array}$ & $\begin{array}{l}39.83 \\
(0.350)\end{array}$ & $\begin{array}{l}\mathrm{r} 33.5 \\
\mathrm{~m} 40.3\end{array}$ & $\begin{array}{l}59.72 \\
(3.67)\end{array}$ & $\begin{array}{l}63.63 \\
(0.182)\end{array}$ & $\begin{array}{l}\mathrm{r} 50.4 \\
\mathrm{~m} 63.7\end{array}$ & $\begin{array}{l}89.34 \\
(4.73)\end{array}$ & $\begin{array}{l}90.37 \\
(0.000)\end{array}$ & $\begin{array}{l}\mathrm{r} 67.4 \\
\text { m } 90.4\end{array}$ \\
\hline$\frac{3}{4}$ & $\begin{array}{l}28.22^{* *} \\
(4.52)\end{array}$ & $\begin{array}{l}35.27 \\
(1.55)\end{array}$ & $\begin{array}{l}\mathrm{r} 21.0 \\
\mathrm{~m} 36.0\end{array}$ & $\begin{array}{l}32.78 \\
(2.06)\end{array}$ & $\begin{array}{l}35.75 \\
(0.567)\end{array}$ & $\begin{array}{l}\mathrm{r} 26.1 \\
\mathrm{~m} 36.2\end{array}$ & $\begin{array}{l}36.13 \\
(0.576)\end{array}$ & $\begin{array}{l}39.52 \\
(0.372)\end{array}$ & $\begin{array}{l}\mathrm{r} 31.0 \\
\mathrm{~m} 40.0\end{array}$ \\
\hline 1 & $\begin{array}{l}36.00 \\
(0.000)\end{array}$ & $\begin{array}{l}36.00 \\
(0.000)\end{array}$ & $\begin{array}{l}\mathrm{r} 13.5 \\
\mathrm{~m} 36.0\end{array}$ & $\begin{array}{l}36.00 \\
(0.000)\end{array}$ & $\begin{array}{l}35.30 \\
(2.29)\end{array}$ & $\begin{array}{l}\mathrm{r} 15.4 \\
\mathrm{~m} 36.0\end{array}$ & $\begin{array}{l}36.00 \\
(0.000)\end{array}$ & $\begin{array}{l}34.48 \\
(2.94)\end{array}$ & $\begin{array}{l}\text { r } 16.6 \\
\text { m } 36.0\end{array}$ \\
\hline
\end{tabular}

Table 3.11: Welfare for Expertise Heterogeneity

not consistently lead to subsequent higher welfare, since for intermediate link specificity welfare is often lower, similarly to the completely homogeneous case. This implies that counterintuitively, enhancing communication in the community by decreasing link specificity is not always an effective tool to increase welfare from communication.

The differences in centralization apparently lead to some tension between overall local efficiency and pairwise stability (until $20 \%$ for $\rho=\frac{3}{4}, \alpha=0$ ). We also look at the individual payoffs in the different parameter settings to study equity issues. For each parameter setting, the average individual payoff and the standard deviation of all agents in the simulated pairwise stable and locally efficient networks are given in Table 3.12.

We thus find that on the individual level there are considerable payoff differences between pairwise stable and locally efficient networks: except for $\rho=\frac{1}{2}, \alpha=1$, the difference between the payoff of the most and least highly valued agents is higher in the pairwise stable networks than in the locally efficient ones. This even holds for $\rho=1$, since as described above, more similarly valuated agents tend to form disjoint pairs with each other in the pairwise stable networks, whereas also other combinations are present in the pair components of the locally efficient networks. Therefore, social preferences would be more beneficial to equity than to efficiency.

\subsubsection{Expertise Heterogeneity with Expert}

For expertise heterogeneity as defined in (3.5), structure and payoff properties of the simulated pairwise stable and locally efficient networks are discussed. 


\begin{tabular}{|c|c|c|c|c|c|c|}
\hline$\rho \backslash \alpha$ & \multicolumn{2}{|c|}{0} & \multicolumn{2}{|c|}{$\frac{1}{2}$} & \multicolumn{2}{|c|}{1} \\
\hline$\frac{1}{2}$ & $\begin{aligned} 1: \begin{array}{l}6.80 \\
(0.038)\end{array} \\
2: \begin{array}{l}7.12 \\
(0.200)\end{array} \\
3: \begin{array}{l}7.60 \\
(0.200)\end{array} \\
4: \begin{array}{l}8.14^{*} \\
(0.043)\end{array} \\
5: \begin{array}{l}6.80 \\
(0.343)\end{array} \\
6: \begin{array}{l}0.000^{* *} * \\
(0.000)\end{array} \\
\end{aligned}$ & 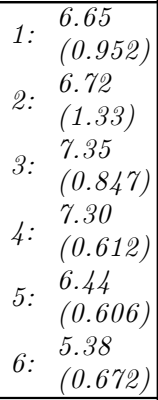 & 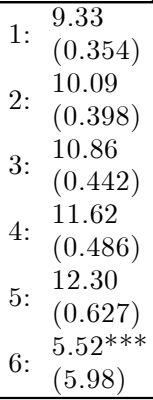 & 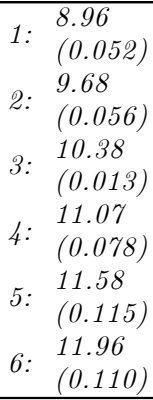 & $\begin{array}{cl}1: & \begin{array}{l}12.59 \\
(0.154)\end{array} \\
2: & \begin{array}{l}13.60 \\
(0.197)\end{array} \\
3: & \begin{array}{l}14.62 \\
(0.240)\end{array} \\
4: & \begin{array}{l}15.63 \\
(0.284)\end{array} \\
5: & \begin{array}{l}16.13 \\
(2.67)\end{array} \\
6: & \begin{array}{l}16.78 \\
(3.45)\end{array} \\
\end{array}$ & 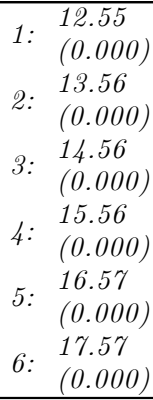 \\
\hline$\frac{3}{4}$ & $\begin{array}{ll}1: \begin{array}{l}7.03^{* * *} \\
(1.74)\end{array} \\
2.54^{* *} \\
(2.15) \\
3: \begin{array}{l}5.60 \\
(0.795)\end{array} \\
4.92^{*} \\
4: \begin{array}{l}1.17) \\
5:\end{array} \\
2.51^{* * *} \\
(1.67) \\
6: \begin{array}{l}1.62^{* * *} \\
(1.50)\end{array}\end{array}$ & 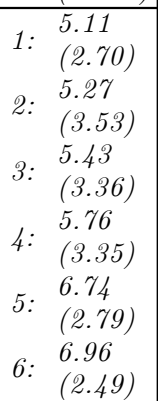 & 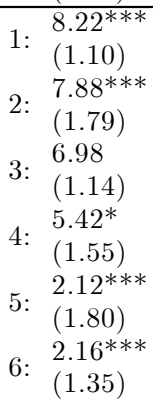 & 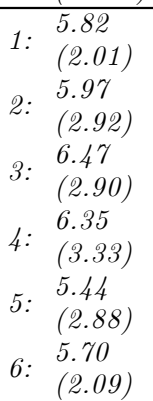 & $\begin{array}{ll}1: & \begin{array}{l}8.85^{* * *} \\
(0.848)\end{array} \\
2: & \begin{array}{l}9.14^{* * *} \\
(1.25)\end{array} \\
3: & \begin{array}{l}8.23^{* * *} \\
(1.97)\end{array} \\
4: & \begin{array}{l}5.44^{* *} \\
(2.42)\end{array} \\
5: & \begin{array}{l}2.88^{* * *} \\
(2.00)\end{array} \\
6: & \begin{array}{l}1.59 * * * \\
(1.50)\end{array}\end{array}$ & $\begin{array}{ll}: & \begin{array}{l}5.49 \\
(0.973)\end{array} \\
2: & \begin{array}{l}5.96 \\
(1.16)\end{array} \\
3: & \begin{array}{l}6.09 \\
(1.18)\end{array} \\
4: & 7.10 \\
(1.25) \\
7.24 \\
5: \\
(0.986) \\
7.64 \\
6: \\
(1.10)\end{array}$ \\
\hline 1 & $\begin{array}{ll}1: & \begin{array}{l}7.32^{* * *} \\
(1.76) \\
8.38^{* * *} \\
2:\end{array} \\
(2.60) \\
3: \begin{array}{l}7.46^{* *} \\
(2.70)\end{array} \\
4: \begin{array}{l}8.04^{* *} \\
(2.76)\end{array} \\
5: \begin{array}{l}1.62^{* * *} \\
(2.01) \\
6:\end{array} \\
3.18^{* * *} \\
(0.572)\end{array}$ & 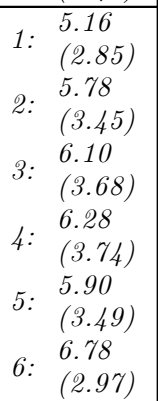 & 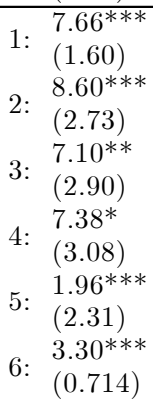 & 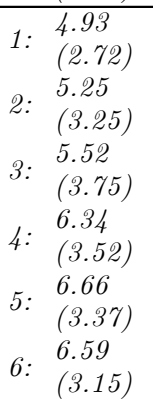 & 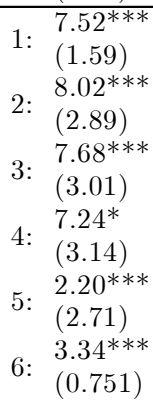 & 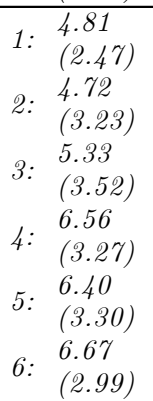 \\
\hline
\end{tabular}

Table 3.12: Payoffs for Expertise Heterogeneity

\section{Structure Properties}

Also for the specific heterogeneous valuation pattern defined in (3.5), the analytical result of Proposition 29 is confirmed by the simulation outcomes. For $\rho=1$ and $\alpha=\frac{1}{2}$ : 68 of the 100 simulated pairwise stable networks consist of the disjoint pair components 1-2, 3-4, and 5-6. Moreover, all other pairwise stable networks also only consist of pairs that do not link the extremely valuated agents to each other: 1-2, 3-5, 4-6 (14); 1-3, 2-4, 5-6 (13); and 1-3, 2-5, 4-6 (5). It is clear that agent 2 even more often than with 
valuation pattern (3.4) forms a pair with the most highly valuated agent.

For each parameter setting, the average density and the standard deviation of the simulated pairwise stable and locally efficient networks are given in Table 3.13 .

\begin{tabular}{|c|l|l|l|l|l|l|}
\hline$\rho \backslash \alpha$ & \multicolumn{2}{|c|}{0} & \multicolumn{2}{c|}{$\frac{1}{2}$} & \multicolumn{2}{c|}{1} \\
\hline$\frac{1}{2}$ & 0.267 & 0.333 & $0.856^{*}$ & 0.717 & 0.977 & 0.920 \\
& $(0.000)$ & $(0.000)$ & $(0.121)$ & $(0.032)$ & $(0.085)$ & $(0.027)$ \\
\hline$\frac{3}{4}$ & 0.200 & 0.245 & 0.200 & 0.263 & $0.200^{*}$ & 0.333 \\
& $(0.000)$ & $(0.031)$ & $(0.000)$ & $(0.016)$ & $(0.000)$ & $(0.000)$ \\
\hline 1 & 0.200 & 0.200 & 0.200 & 0.202 & 0.200 & 0.209 \\
& $(0.000)$ & $(0.000)$ & $(0.000)$ & $(0.011)$ & $(0.000)$ & 0.022 \\
\hline
\end{tabular}

Table 3.13: Densities for Expertise Heterogeneity with Expert

The density values thus are very low due to the combination of expertise heterogeneity and expert: for $\rho=\frac{3}{4}, 1$ the pairwise stable networks only consist of disjoint pairs. Furthermore, the basic intuition that increasing $\rho$ implies decreasing density is partly confirmed by the simulation outcomes as well as the intuition about the positive effect of $\alpha$ on density.

For each parameter setting, the average relative centrality and the standard deviation of all agents in the simulated pairwise stable and locally efficient networks are given in Table 3.14.

Similarly to the case without expert, decreasing $\rho$ leads to more centrality for the expert and less centrality for the lowest valued agent first, after which the density increases further such that these centralities become closer again. Also $\alpha$ again does not have a consistent effect on expert centrality.

We find that the centralization of the expert is no longer noteworthily higher in the pairwise stable than in the locally efficient networks as in Section 3.5.3, but other differences between pairwise stable and locally efficient structures can be identified: in most cases the difference in relative centralization between most and least highly valued non-experts is higher for the pairwise stable networks $\left(\rho=\frac{1}{2} ; \alpha=0, \frac{1}{2}\right.$ and $\rho=\frac{3}{4}$ ), like in the case of heterogeneity without expert (Table 3.9). In the following example we illustrate one of these cases. 


\begin{tabular}{|c|c|c|c|c|c|c|}
\hline$\rho \backslash \alpha$ & \multicolumn{2}{|c|}{0} & & \multicolumn{2}{|l|}{1} \\
\hline & 0.925 & 1.00 & $0.054^{*}$ & 0.199 & 1. 0.007 & 1.0.044 \\
\hline & $(0.159)$ & 1. $(0.000)$ & 1. $\quad(0.029)$ & (0.032) & $(0.026)$ & $(0.016)$ \\
\hline & -0.125 & -0.200 & 0.054 & 0.066 & 0.007 & 0.044 \\
\hline & $2: \quad(0.000)$ & $2: \quad(0.000)$ & 2: $\quad(0.029)$ & $2: \quad(0.057)$ & $2: \quad(0.026)$ & $(0.016)$ \\
\hline & -0.125 & -0.200 & 0.039 & -0.015 & 0.007 & 0.021 \\
\hline & $3: \quad(0.000)$ & $3: \quad(0.000)$ & $(0.053)$ & $3: \quad(0.059)$ & $3: \quad(0.026)$ & $(0.030)$ \\
\hline & -0.121 & -0.200 & 0.034 & -0.037 & 0.007 & 0.021 \\
\hline & 4: $\quad(0.037)$ & 4: $(0.000)$ & 4: $\quad(0.058)$ & 4: $\quad(0.057)$ & 4: $\quad(0.026)$ & $(0.030)$ \\
\hline & -0.125 & -0.200 & 5. 0.014 & 5. -0.078 & 5. 0.007 & -0.065 \\
\hline & 5: $\quad(0.000)$ & $3: \quad(0.000)$ & $(0.071)$ & $3: \quad(0.021)$ & $5: \quad(0.026)$ & $(0.013)$ \\
\hline & 6. $-0.429^{*}$ * & 6. -0.200 & 6. -0.196 & 6. -0.135 & 6. -0.035 & -0.065 \\
\hline & 6: $\quad(0.147)$ & $(0.000)$ & $(0.194)$ & $(0.056)$ & $(0.128)$ & $(0.013)$ \\
\hline & $0.395^{*}$ & 0.295 & 0.425 & 0.370 & 0.500 & 0.433 \\
\hline & 1. $\quad(0.204)$ & 1. (0.258) & I: $\quad(0.179)$ & 1: $\quad(0.200)$ & 1: $\quad(0.000)$ & $(0.094)$ \\
\hline & 2. 0.000 & ๑. -0.009 & 2. 0.000 & ๑. -0.054 & 2. 0.000 & ๑. -0.029 \\
\hline & $2: \quad(0.000)$ & : (0.141) & 2: $\quad(0.000)$ & $2: \quad(0.141)$ & 2: $\quad(0.000)$ & $(0.149)$ \\
\hline & 3. 0.000 & -0.061 & 3. 0.000 & 3. -0.058 & 3. 0.000 & -0.074 \\
\hline & $3: \quad(0.000)$ & $(0.098)$ & $3: \quad(0.000)$ & $3: \quad(0.137)$ & $(0.000)$ & $(0.148)$ \\
\hline & 4. 0.000 & -0.065 & 4. 0.000 & 1. -0.069 & 4. 0.000 & . -0.059 \\
\hline & 4: $\quad(0.000)$ & (0.093) & $(0.000)$ & 4: $\quad(0.127)$ & $(0.000)$ & $(0.150)$ \\
\hline & 5: $\quad 0.000$ & -0.076 & 5: $\quad 0.000$ & 5: $\quad-0.076$ & 5: $\quad 0.000^{*}$ & -0.137 \\
\hline & (0.000) & $(0.075)$ & $(0.000)$ & $(0.118)$ & $(0.000)$ & (0.122) \\
\hline & 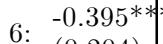 & -0.084 & $6:-0.425^{\text {* }}$ 将 & $6:-0.114$ & $6:-0.500^{* *}$ & -0.134 \\
\hline & $0: \quad(0.2$ & $(0.059)$ & 0: $\quad(0.179)$ & $0: \quad(0.047)$ & $(0.000)$ & $(0.124)$ \\
\hline & 0.000 & 0.000 & 0.000 & 1.0.093 & 0.000 & 0.063 \\
\hline & 1: $\quad(0.000)$ & $1 . \quad(0.000)$ & 1: $\quad(0.000)$ & $1 . \quad(0.189)$ & 1: $\quad(0.000)$ & (0.139) \\
\hline & 2. 0.000 & 0.000 & 2. 0.000 & -0.004 & 0.000 & -0.001 \\
\hline & $2: \quad(0.000)$ & $2: \quad(0.000)$ & 2: $\quad(0.000)$ & (0.021) & $2: \quad(0.000)$ & $(0.062)$ \\
\hline & 3. 0.000 & ฉ. 0.000 & 0.000 & -0.004 & 0.000 & -0.001 \\
\hline & $3: \quad(0.000)$ & $3: \quad(0.000)$ & $3: \quad(0.000)$ & $3: \quad(0.021)$ & $(0.000)$ & (0.062) \\
\hline & 4. 0.000 & 0.000 & 0.000 & -0.004 & 0.000 & -0.016 \\
\hline & 4: $\quad(0.000)$ & 4: $(0.000)$ & 4: $\quad(0.000)$ & 4: $\quad(0.021)$ & 4: $\quad(0.000)$ & $(0.042)$ \\
\hline & 5. 0.000 & 5. 0.000 & 5. 0.000 & -0.004 & 5. 0.000 & -0.016 \\
\hline & S: $\quad(0.000)$ & $3: \quad(0.000)$ & $(0.000)$ & $(0.021)$ & $(0.000)$ & $(0.042)$ \\
\hline & 6. 0.000 & 6. 0.000 & 6. 0.000 & 6. -0.078 & 6. 0.000 & -0.028 \\
\hline & $(0.000)$ & $0: \quad(0.000)$ & b: $\quad(0.000)$ & $0: \quad(0.196)$ & $(0.000)$ & $(0.136)$ \\
\hline
\end{tabular}

Table 3.14: Centralities for Expertise Heterogeneity with Expert

Example 31 Let $\rho=\frac{3}{4}, \alpha=\frac{1}{2}$, then Table 3.15 provides an overview of the types and numbers of pairwise stable networks resulting from 100 simulations and the types and numbers of locally efficient networks resulting from another 100 simulations.

Thus, in the pairwise stable networks agent 6 is relatively isolated, whereas in the locally efficient networks she is more equally centralized to the other non-experts. 


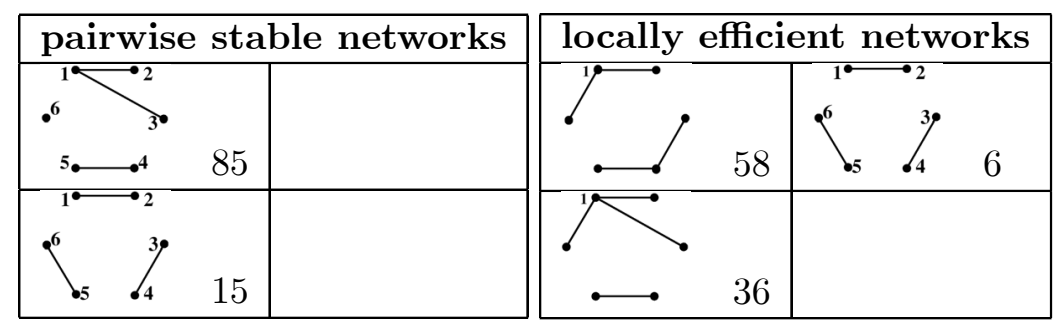

Table 3.15: Example of Structures for Expertise Heterogeneity with Expert

\section{Payoff Properties}

As a means to investigate in how far the structure differences between the locally efficient and pairwise stable networks actually lead to welfare differences, for each parameter setting, the average welfare and the standard deviation of the simulated pairwise stable and locally efficient networks are given in Table 3.16 .

\begin{tabular}{|c|l|l|l|l|l|l|l|l|l|}
\hline$\rho \backslash \alpha$ & \multicolumn{3}{|c|}{0} & \multicolumn{3}{c|}{$\frac{1}{2}$} & \multicolumn{3}{c|}{1} \\
\hline$\frac{1}{2}$ & $48.45^{*}$ & 53.67 & $\mathrm{r} \mathrm{33.5}$ & 61.60 & 64.46 & $\mathrm{r} \mathrm{50.0}$ & 89.48 & 90.42 & $\mathrm{r} 67.1$ \\
& $(1.20)$ & $(0.000)$ & $\mathrm{m} 53.7$ & $(3.37)$ & $(0.142)$ & $\mathrm{m} 64.8$ & $(3.245)$ & $(0.130)$ & $\mathrm{m} 90.5$ \\
\hline$\frac{3}{4}$ & 35.47 & 36.87 & $\mathrm{r} 21.1$ & 36.78 & 38.40 & $\mathrm{r} 26.1$ & 38.51 & 41.27 & $\mathrm{r} 31.1$ \\
& $(0.275)$ & $(1.11)$ & $\mathrm{m} 39.3$ & $(0.327)$ & $(1.13)$ & $\mathrm{m} 40.4$ & $(0.000)$ & $(0.466)$ & $\mathrm{m} 42.2$ \\
\hline 1 & 36.00 & 36.00 & $\mathrm{r} 13.7$ & 36.00 & 35.18 & $\mathrm{r} 15.2$ & 36.00 & 35.48 & $\mathrm{r} 16.9$ \\
& $(0.000)$ & $(0.000)$ & $\mathrm{m} 36.0$ & $(0.000)$ & $(1.66)$ & $\mathrm{m} 36.0$ & $(0.000)$ & $(1.10)$ & $\mathrm{m} 36.0$ \\
\hline
\end{tabular}

Table 3.16: Welfare for Expertise Heterogeneity with Expert

Except for $\rho=1$, we again find that a higher $\alpha$ and thus more value transferability leads to higher welfare. Therefore, also in a heterogeneous setting with an expert, stimulating the focus on informational value in the community is an effective tool for boosting welfare. Furthermore, also in this setting a lower $\rho$ only leads to subsequent higher welfare when link specificity is low enough.

The structure differences do only lead to a small tension between overall local efficiency and pairwise stability for $\rho=\frac{1}{2}, \frac{3}{4}$ (until $10 \%$ for $\rho=\frac{1}{2}$, $\alpha=0$ ). Comparing Tables 3.11 and 3.16, adding an expert to a heterogeneous community appears to be a less effective tool to increase welfare for intermediate link specificity than in case of a homogeneous community. 
Again we also look at the individual payoffs in the different parameter settings to study equity issues. For each parameter setting, the average individual payoff and the standard deviation of all agents in the simulated pairwise stable and locally efficient networks are given in Table 3.17.

\begin{tabular}{|c|c|c|c|c|c|c|}
\hline$\rho \backslash \alpha$ & \multicolumn{2}{|c|}{0} & \multicolumn{2}{|c|}{$\frac{1}{2}$} & \multicolumn{2}{|c|}{1} \\
\hline$\frac{1}{2}$ & $\left.\begin{array}{ll}1: & \begin{array}{l}6.98 \\
(0.068)\end{array} \\
2: & \begin{array}{l}10.84^{*} \\
(0.742)\end{array} \\
3: & \begin{array}{l}10.84^{*} \\
(0.742)\end{array} \\
4: & 10.71^{*} \\
(1.06) \\
5:\end{array}\right) \begin{array}{l}8.71 \\
(3.71) \\
0.381^{*} * * \\
6: \\
(0.787)\end{array}$ & 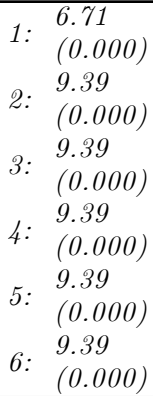 & 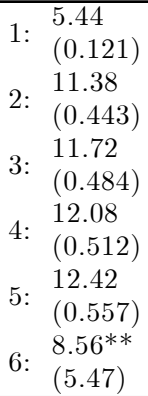 & 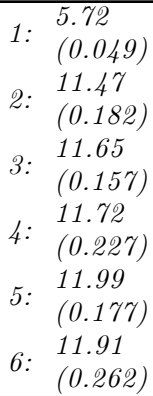 & $\begin{array}{ll}1: & \begin{array}{l}7.55 \\
(0.060)\end{array} \\
2: & \begin{array}{l}15.64 \\
(0.275)\end{array} \\
3: & \begin{array}{l}16.15 \\
(0.288)\end{array} \\
4: & 16.65 \\
& (0.301) \\
5: & 17.16 \\
& (0.315) \\
6: & 16.34 \\
& (4.48)\end{array}$ & 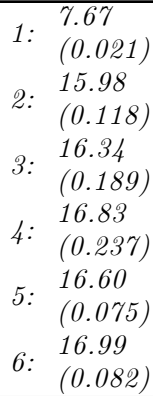 \\
\hline$\frac{3}{4}$ & 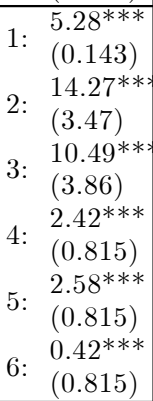 & 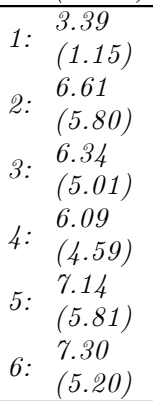 & 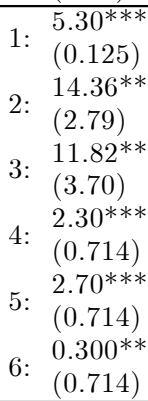 & 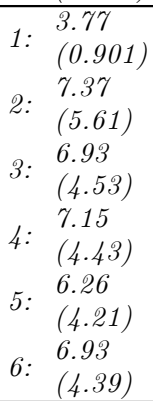 & 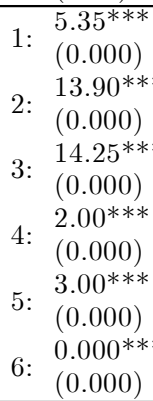 & 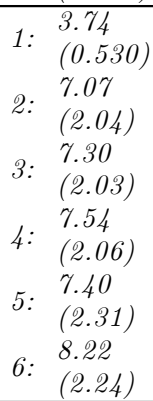 \\
\hline 1 & 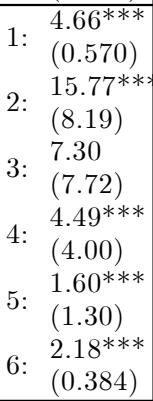 & 1: $\begin{array}{l}3.39 \\
(1.36) \\
2.00 \\
2: \\
(8.37) \\
3: \begin{array}{l}6.82 \\
(7.44)\end{array} \\
4: \begin{array}{l}6.95 \\
(7.58)\end{array} \\
5: \begin{array}{l}5.48 \\
(6.90)\end{array} \\
6: \begin{array}{l}5.36 \\
(5.88)\end{array}\end{array}$ & 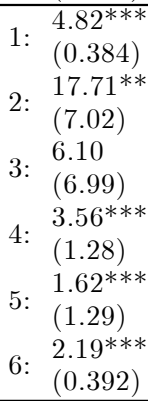 & 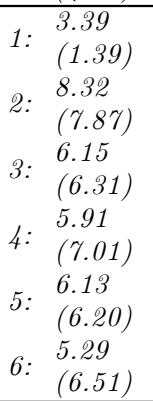 & 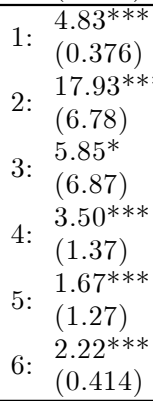 & $\begin{array}{ll}: \begin{array}{l}3.04 \\
(1.30)\end{array} \\
2: \begin{array}{l}5.58 \\
(6.64)\end{array} \\
3: \begin{array}{l}7.06 \\
(7.40)\end{array} \\
4: \begin{array}{l}7.10 \\
(7.01)\end{array} \\
5: \begin{array}{l}6.28 \\
(6.30) \\
6.42 \\
6: \\
(6.80)\end{array}\end{array}$ \\
\hline
\end{tabular}

Table 3.17: Payoffs for Expertise Heterogeneity with Expert

We find that on the individual level there are considerable payoff differences between pairwise stable and locally efficient networks: except for $\rho=\frac{1}{2}, \alpha=1$, the difference between the payoff of the most and least highly valued non-experts is much higher in the pairwise stable networks than in the locally efficient ones. Therefore, also with expert social preferences would 
be more beneficial to equity than to efficiency. Moreover, comparing Tables 3.12 and 3.17, adding an expert to a heterogeneous community does by far not resolve inequity. This can be explained by the effect that in case of expertise heterogeneity, more similarly valuated agents tend to form disjoint pairs with each other, which leads to an extreme payoff for the agent who is most highly valuated after the expert. Only in case of higher density (due to small link specificity plus value transferability), all agents benefit from the presence of the expert.

\subsection{Discussion}

In this chapter we studied the structure and efficiency of bilateral communication links by simulating a game-theoretic model of network formation for three levels of link specificity - the extent that having to maintain more direct links leads to each link's decrease in value -, three levels of focus on informational versus social value from communication, and four patterns of valuation heterogeneity: homogeneity and expertise heterogeneity, each with and without the presence of one especially highly valued expert. We have illustrated that the structure properties of pairwise stable networks heavily depend on these parameter values, which results in efficiency and equity differences among settings. Therefore, in this section we open the discussion on measurement and management of these important parameters.

\subsubsection{Measurement}

First of all it is important for a manager to assess the valuation pattern in her community. For example, the effects of adding an expert to a community depend on whether the rest of the community is homogeneous (Section 3.5.3) or not (Section 3.5.5). An obvious way to measure this is via a credit point or ranking system as present in many online communities nowadays (e.g., hp's IT resource center forums). A complicating factor of such a system is that the possibility to judge other members' performance might itself be a cause 
of heterogeneity, for it visualizes and stimulates some members' performance efforts. Therefore, it may be necessary to derive the level of heterogeneity from more objective community measures like message exchange or to send out a questionnaire to a random sample from the members.

Furthermore, the level of focus on informational versus social value from communication $(\alpha)$ is crucial for payoff properties of the resulting network structure. In this chapter, where $V_{i, j}^{\mathrm{i}}=V_{i, j}^{\mathrm{s}} \forall i, j \in N: i \neq j$, more focus on informational value was generally beneficial for community welfare. However, we know from Chapter 2 that this is no longer guaranteed for any ratio between informational and social value (Section 2.4.3). Also for this, parameter estimates might be derived from objective community statistics like the use of common technical terms (e.g., numbers) versus the use of common social support terms (e.g., emoticons), or via a questionnaire.

Finally, the level of specificity in the community is a managerially relevant parameter. It may be relatively easy to determine whether specificity is higher in one community or another, since it is possible to compare via message exchange or a questionnaire how much effort members have to put into making their contributions valuable for specific other members. Probably it is more difficult to estimate absolute values of the parameter $\rho$, reflecting how exactly these effort levels affect payoffs when members have to maintain an increasing number of links. However, it is important for a manager to know this, because for example in Section 3.5.2 it was illustrated that enhancing communication in the community by decreasing link specificity does not always lead to higher welfare from communication. A solution could be to find the current level of $\rho$ - after measuring the other important parameter values - by backward induction from the structure properties of the community network. E.g., for a homogeneous valuation pattern, complete structures point out a $\rho$ value of at most $\frac{1}{2}$ and fragmented pair structures a $\rho$ value of at least 1 . 


\subsubsection{Management}

Depending on the measurement issues as discussed above, the current chapter suggests several community management tools to affect the value that members derive from communication.

As already discussed in Section 2.4.3 of Chapter 2, the informational versus social orientation $(\alpha)$ could be influenced by modifying moderator tasks, entry policy, rules of conduct, and reward systems, hereby emphasizing informational versus social value.

By reward systems and especially by adding an expert to the community, managers can affect the value derived from communication effectively via the community's valuation pattern. Also, there may be ways to influence $\rho$, for example by technically simplifying the process of dealing with multiple communication links.

The local efficiency results of Section 3.5 illustrated that in several parameter settings, social preferences in the sense that members use community welfare as their objective function rather than their own payoff can enhance welfare or equity to a consequential extent. Therefore, in Chapter 4 it will be investigated whether people take others' payoff into account when making network formation decisions.

\subsubsection{Further Research}

These simulation studies furthermore illustrate the interest of economic, structure analysis of communication. Follow-up studies can investigate the effects of an agent who is given incentives by the community manager to serve welfare rather than own payoff. Preliminary simulations indicate that this will lead to more efficient pairwise stable networks, even if this special agent is not an expert in the sense that other community members derive more value from communication with her.

Moreover, more complex forms of valuation heterogeneity can be explored. As already mentioned before, the condition $V_{i, j}^{\mathrm{i}}=V_{i, j}^{\mathrm{s}} \forall i, j \in N$ : 
$i \neq j$ could be dropped, resulting in other effects of $\alpha$. Also, besides expertise heterogeneity as studied here - agents represent different values for their fellow customers - it is possible to study judgement heterogeneity, where agents have different opinions on the values of their fellow customers. Preliminary simulations indicate that this leads to agent permutations rather than structure differences. For example, if $\rho=1$, the pairwise stable structures in case of judgement heterogeneity still only consist of pairs, but the likelihood that agents 1 and 2 form one of these pairs changes.

In addition, it is possible to derive more general analytical results like in Chapter 2 also for $\rho$-values other than 1 . In particular for the other extreme case simulated here, i.e., $\rho=\frac{1}{2}$, it would be interesting to characterize the conditions in which the complete network is the only pairwise stable network.

The managerial implication practice of affecting parameters of the model might bring up other ways of controlling the welfare and equity of communities asking for further research. Empirical testing forms an alternative promising area. 


\section{Chapter 4}

\section{The Behavioral Aspect: An Experimental Investigation}

Network formation constitutes an important part of many social and economic processes, but relatively little is known about how individuals make their linking decisions. This chapter provides an experimental investigation of behavioral effects in individual decisions of network formation. Our findings demonstrate that individuals systematically simplify more complex components of network payoff in their linking decisions. Specifically, they focus on only part of the normative payoff, namely on their own direct payoff and tend to ignore indirect payoff and payoff for others in the network. Additionally, individuals use descriptive behavioral traits of link choice alternatives to guide their choices. They are sensitive to whether an alternative involves link deletion or creation and whether it concerns an isolated or a central node. Furthermore, we find that complexity of one type can moderate individuals' dealing with a complex feature of another type. These behavioral effects have important implications for researchers and managers working in areas that involve network formation. 


\subsection{Introduction}

Network formation among individuals is an important phenomenon in many social and economic contexts, ranging from word-of-mouth communications among consumers (e.g., Iacobucci and Hopkins 1992) and social structure (e.g., Granovetter 1995) to intra-organization communication (e.g., Van den Bulte and Moenaert 1998) and virtual communities (e.g., Wellman et al. 1996).

There exists a recent and increasing literature in which researchers experimentally investigate the network formation process. One stream in this literature is involved with testing integral game-theoretical models of network formation. They include variants of Bala and Goyal's (2000) noncooperative network formation model (e.g., Berninghaus et al. 2006, Callander and Plott 2005), Jackson and Wolinsky's (1996) pairwise cooperative network formation model (e.g., Deck and Johnson 2004), and fully cooperative network formation models like Jackson and Van den Nouweland's (2005) (e.g., Charness and Jackson 2006). This research identifies several conditions under which theoretically stable network structures are reproduced in the laboratory and addresses these networks' efficiency. Another stream of experimental studies examines the role of network formation as endogenously emerging in other relevant settings of cooperative decision making (e.g., Brown et al. 2004, Corbae and Duffy 2007, Hauk and Nagel 2001, Kirchsteiger et al. 2005). This research shows that cooperation decisions are considerably influenced when individuals are allowed to choose their partners versus when an interaction structure is imposed.

A common factor in this previous empirical investigation of the network formation process is that individual benefits and costs are given by a payoff function tailored to the specific game-theoretical setting. Therefore, an issue that has been largely ignored is that the complexity of the network formation decisions that individuals face may cause errors or simplifications in their choice process. Although previous research acknowledged the mere existence of errors (e.g., McKelvey and Palfrey 1995, Charness and Jackson 2006), 
the underlying process remains undisclosed. The objective of the current chapter is to investigate such behavioral shifts in individual decision making in networks as a function of typical network complexity features. In particular, we examine whether individuals use simplifying heuristics in dealing with network complexity, similarly to how they use simplifying heuristics in previously studied choice contexts (Johnson and Payne 1985).

We perform an individual decision making experiment in which we vary three complexity features that are relevant in the context of network formation, i.e., structural complexity of the network, transferability of value in the network, and social tradeoff between one's own payoff and others' payoff. These properties complicate the choices that individuals make about creating and maintaining links in the network. We examine whether they affect individuals' use of simplifying heuristics. We investigate whether complex network features are represented in a simplified form in the individual decision process, and how complexity of one type can moderate the individual handling of a complex feature of another type.

In general, we investigate whether individuals cope with complexity by attribute substitution, i.e., assessing the target attribute of a judgment object by substituting a property of the object that is more accessible, i.e., comes more readily to mind (Gilovich et al. 2002, Kahneman 2003). Individuals may thus simplify the network formation decision process in two ways. First, they substitute the exact payoff value of a link choice alternative by descriptive attributes that are related to this value, e.g., whether a linking alternative involves link deletion or creation and whether it concerns an isolated or a central node. Second, they focus relatively strongly on the value they derive from their direct neighbors rather than indirect neighbors and they put less weight on the payoffs that others receive. We examine whether these two types of heuristics are more heavily used in more complex settings.

In order to test our hypotheses, we confront participants in the lab with multiple static, non-interactive network situations in which they can choose to create or delete one link or to do nothing. Such a network situation 
constitutes one of the simplest network linking decision contexts, which allows us to study the effects of network complexity under highly controlled experimental conditions. In more complex network tasks, like the strategic situations as commonly studied in the current experimental network formation literature, the proposed complexity effects are also likely to play a significant role, but it would be much harder to disentangle each component's separate effect.

The participants' choices have a direct impact on their monetary rewards in the experiment and differ with respect to the three complexity factors (structural complexity, value transferability, social tradeoff), leading to different treatments. We perform a comprehensive parametric test of the hypotheses by estimating a mixed (i.e., random parameters) logit model (McFadden 2001, Hensher et al. 2005). This allows us to investigate the impact of important complex network properties on individuals' decisions, while allowing for heterogeneity of the decision makers.

In Section 4.2, we present our theoretical framework, leading to hypotheses on behavioral effects in network linking decision making that differ from prior predictions based on full rationality. Section 4.3 describes the experimental design and the approach used for the mixed logit estimations. The results of our experiment and hypotheses tests are reported in Section 4.4. Section 4.5 concludes the chapter with discussion.

\subsection{Theoretical Framework}

The objective of this section is to present our hypotheses about behavioral effects in individual decisions of network formation and compare them to predictions on individual choice behavior underlying the previous experimental network formation literature. After a description of our setting, the predictions based on prior theories are reviewed in Section 4.2.1 and our hypotheses are presented in Section 4.2.2.

The focus of our research is to investigate individuals' behavioral res- 
ponse to variations in network complexity. We address the elementary case of single link formation decisions by individuals. Doing so allows us to investigate complexity effects in a tightly controlled yet relevant setting of network formation decisions. To prevent possible confounding effects that do not originate from network complexity but from strategic interaction among individuals, we focus on individual one-period decisions. Thus a typical decision task as we study it would be described as follows. An individual ("you" in the example of Figure 4.1) is connected with several other nodes in a network and is facing the one-shot choice problem to change at most one link: her options are to delete one of her existing links (a or $\mathrm{d}$ in the example), to create a link with one node that she is currently not directly connected to ( $\mathrm{b}$ or $\mathrm{c}$ in the example), or not to change anything. Her payoff entirely depends on the network structure after completion of her choice.

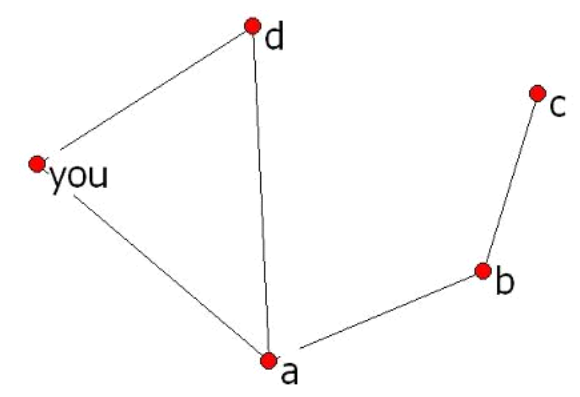

Figure 4.1: Example

\subsubsection{Prior Utility Models of Network Formation Decisions}

Economic theory traditionally models the utility that individual $i$ derives from choosing alternative $j$ as:

$$
U_{j}^{i}=f^{i}\left(\operatorname{Payoff}_{j}^{i}\right)
$$

where Payoff ${ }_{j}^{i}$ is the payoff experienced by individual $i$ when she chooses alternative $j$ and $f^{i}$ is a strictly increasing function. 
Social preferences theory (for an overview see Fehr and Schmidt 2003) augments this model by allowing for the fact that in addition to their own payoff, individuals may take the payoff for other individuals into account when making their decisions. In this case, the utility that individual $i$ derives from choosing alternative $j$ is given by:

$$
U_{j}^{i}=f^{i}\left(\text { OwnPayoff }_{j}^{i},\left(\text { OthersPayoff }(q)_{j}^{i}\right)_{q \neq i}\right),
$$

where OwnPayoff ${ }_{j}^{i}$ is the payoff personally experienced by individual $i$ when she chooses alternative $j$, OthersPayoff $(q)_{j}^{i}$ is the payoff experienced by another individual $q$ when individual $i$ chooses alternative $j$, and $f^{i}($.$) is a$ function reflecting how individual $i$ holds others-oriented payoff components in mind (e.g., inequity aversion, efficiency preferences, etc.).

\subsubsection{Hypotheses}

Our claim is that these prior utility models are not sufficient in explaining link choice behavior in the presence of network complexity. As humans have bounded rationality (Loasby 1976, Simon 1979, Camerer 1998), they cope with complexity in decision making by using simplifying heuristics, which commonly involve assessing a judgment object (e.g., linking choice alternative) using only the subset of properties of the object that are most accessible, i.e., that come most readily to mind, rather than using all relevant properties (Gilovich et al. 2002, Kahneman 2003, Gigerenzer et al. 1999). For example, when faced with a choice that is hard to resolve, people often search for a compelling rationale for choosing one alternative over another (Shafir et al. 1993). This is clearly illustrated in the literature about the effects of task complexity in several other contexts, like audit judgment (e.g., Bonner 1994) and consumer choice (e.g., Swait and Adamowicz 2001), but no research to date has addressed simplification rules that individuals use in making complex network formation decisions.

In the next subsections, we propose two main types of simplifying heuristics that individuals use in making linking decisions: (i) they employ de- 
scriptive properties of the choice alternatives besides their normative payoff properties and (ii) they focus on only the most easily accessible parts of the normative payoff properties. Furthermore, we examine whether both forms of simplification are stronger under more complex network decision making conditions.

\section{Descriptive Traits}

In the network formation setting the decision maker's payoff depends on the network structure after completion of her choice, where having more connections is on the one hand beneficial, since they provide access to additional resources, and on the other hand costly, for it takes time and effort to maintain them. It is typically a complex task for individuals to judge the exact payoff of link choice alternatives and we examine whether individuals use simplifying heuristics. A similar psychological process of judgment simplification is encountered in the literature about Conjunctive Probability Assessment, which shows that individuals make predictions based on a correlation they assume to exist between the assessment variable and some other variable (e.g., Broniarczyk \& Alba 1994).

Accordingly, we investigate whether individuals partly substitute the normative payoff value of a link choice alternative by descriptive attributes that can be determined more easily and that are qualitatively related to it. In particular, individuals can relatively easily see that there is a tradeoff between benefits and costs of more connections. Consequently, we hypothesize that they use as heuristic attributes (i) whether a link choice alternative involves link deletion or creation, and (ii) whether it concerns an isolated node, i.e., a node without direct links, or a central node, i.e., a node with three or more direct links. This is in line with Qualitative Process Theory, which suggests that human reasoning is more likely to depend on qualitative rather than quantitative relations (Forbus 1993). Therefore, it is examined whether individuals use the type of action or node as behavioral cues rather than the expected payoff. 
Summarizing, we can formulate the following hypothesis:

Hypothesis 1 (effects of descriptive traits on link choice) An individual's network formation choices are affected by descriptive traits of link choice alternatives, i.e., whether an alternative involves link deletion (-) or creation $(+)$, and whether it concerns an isolated node $(+)$ or a central node $(-)$.

Thus, the hypothesized effects of creation and deletion are based on the qualitative benefits of links, whereas the hypothesized effects of isolated and central nodes are based on the qualitative costs of links. Pursuing this line of reasoning, we formulate the utility that individual $i$ derives from choosing alternative $j$ with the following behavioral cues:

$$
U_{j}^{i}=f^{i}\left(\text { Payoff }_{j}^{i}, \text { Delete }_{j}, \text { Create }_{j}, \text { Central }_{j}, \text { Isolated }_{j}\right),
$$

where Payoff ${ }_{j}^{i}$ is the payoff experienced by individual $i$ when she chooses alternative $j$, and Delete $j$, Create $_{j}$, Central $_{j}$, and Isolated $_{j}$ are dummy variables indicating by 1 that alternative $j$ involves link deletion, link creation, a link with a central node, and a link with an isolated node, respectively. The function $f^{i}$ decreases in Delete ${ }_{j}$ and $\mathrm{Central}_{j}$, and increases in Create ${ }_{j}$ and Isolated $_{j}$.

\section{Shifts in Payoff Traits}

Direct versus Indirect Payoff An important aspect of complexity in a network setting is that there can be value transferability, implying that an individual derives value not only from her direct neighbors, but also from her neighbors' neighbors, etc. (e.g., Bala and Goyal 2000, Jackson and Wolinsky 1996). This makes it more complex for individuals to judge the exact value of link choice alternatives, because besides evaluating the direct payoff of each link, they also have to be forward-looking over indirect links. 
Our hypothesis is that individuals tend to simplify by focusing most strongly on the payoff aspect that can be determined most easily: the value that is derived from direct neighbors only. The tendency to discount items in the utility function that are relatively more distant is known from research about time discounting (e.g., Frederick et al. 2002). Closely related to our hypothesis of network-shortsightedness are findings of spatial discounting, i.e., weighting spatially close features more strongly in the utility function than spatially distant features (e.g., Hannon 1994, Levinthal and March 1993).

Therefore, we hypothesize:

Hypothesis 2 (effects of indirect payoff on link choice) An individual's network formation choices are affected less strongly by payoff she derives from indirect nodes $(+)$ than by payoff from direct nodes $(+)$.

We capture this second proposed behavioral effect as follows in the utility that individual $i$ derives from choosing alternative $j$ :

$U_{j}^{i}=f^{i}\left(\right.$ DirectPayoff $_{j}^{i}$, IndirectPayoff $_{j}^{i}$, Delete $_{j}$, Create $_{j}$, Central $_{j}$, Isolated $\left._{j}\right)$,

where DirectPayoff ${ }_{j}^{i}$ is the payoff experienced by individual $i$ from her direct neighbors when she chooses alternative $j$, IndirectPayoff ${ }_{j}^{i}=\operatorname{Payoff}_{j}^{i}-$ DirectPayoff ${ }_{j}^{i}$, and $f^{i}$ increases in IndirectPayoff ${ }_{j}^{i}$ but more so in DirectPayoff ${ }_{j}^{i}$.

Own versus Others' Payoff Social tradeoff is a further complicating factor in the network setting, implying that an individual's choices not only affect her own value, but also her neighbors' (and her neighbors' neighbors, etc.) (e.g., Bala and Goyal 2000, Jackson and Wolinsky 1996). This aspect of network formation choices makes it more complex for individuals with social preferences to judge the exact value of link choice alternatives, because besides their own payoff they also have to consider the payoff of (possibly many) other individuals. 
Similarly to our investigation with respect to how individuals simplify the complexity of having to deal with indirect payoff, we also investigate whether individuals deal with the complexity of social tradeoff by focusing on the payoff aspect that can be determined most easily. In this case we examine whether individuals tend to focus more strongly on their own payoff and will pay relatively less attention to others' payoff due to the greater complexity of evaluating this social payoff. In the past, behavioral economists have found empirical evidence for social preferences, but mainly in lab environments where choice complexity was largely mitigated by explicit payoff information, which presents participants with the numerical payoff consequences of their choice options. However, Charness et al. (2004) have shown that individuals' social choices are affected by the variation of complexity resulting from such a comprehensive payoff overview.

Thus, we propose the following hypothesis:

Hypothesis 3 (effects of others' payoff on link choice) An individual's network formation choices are affected less strongly by their payoff consequences for other individuals $(+)$ when she is provided with a complete description of how the payoffs depend on these choices than when she is provided with a numerical payoff overview $(+)$.

We include this third behavioral effect in the utility that individual $i$ derives from choosing alternative $j$ as follows:

$$
\begin{aligned}
& U_{j}^{i}=f^{i} \text { (OwnDirectPayoff }{ }_{j}^{i} \text {, OwnIndirectPayoff }{ }_{j}^{i}, \\
& \text { (OthersDirectPayoff } \left.(q)_{j}^{i}\right)_{q \neq i},\left(\text { OthersIndirectPayoff }(q)_{j}^{i}\right)_{q \neq i} \text {, } \\
& \text { Delete } \left._{j}, \text { Create }_{j}, \text { Central }_{j}, \text { Isolated }_{j}\right) \text {, }
\end{aligned}
$$

where OwnDirectPayoff ${ }_{j}^{i}$ is the payoff personally experienced by individual $i$ from her direct neighbors when she chooses alternative $j$, OwnIndirectPayoff ${ }_{j}^{i}$ is the payoff personally experienced from indirect nodes, OthersDirectPay$\operatorname{off}(q)_{j}^{i}$ is the payoff experienced by another individual $q$ from her direct 
neighbors when individual $i$ chooses alternative $j$, OthersIndirectPayoff $(q)_{j}^{i}$ is the payoff experienced by another individual from indirect nodes, and $f^{i}$ is a function less increasing in (OthersPayoff $\left.(q)_{j}^{i}\right)_{q \neq i}$ when individual $i$ does not receive an explicit payoff overview than when she does.

\section{Complexity Conditions}

Finally, we hypothesize that the different complexity aspects of networks may strengthen individuals' tendency to rely on the proposed heuristics. We include these moderating effects of complexity in the utility that individual $i$ derives from choosing alternative $j$ as follows:

$$
\begin{aligned}
U_{j}^{i}= & f^{i}\left(\text { OwnDirectPayoff }_{j}^{i}, \text { OwnIndirectPayoff }_{j}^{i},\right. \\
& \left(\text { OthersDirectPayoff }_{(q)}^{i}\right)_{q \neq i},\left(\text { OthersIndirectPayoff }_{q}(q)_{j}^{i}\right)_{q \neq i}, \\
& \text { Delete }_{j}, \text { Create }_{j}, \operatorname{Central}_{j}, \text { Isolated }_{j}, \\
& \text { Complexity }^{i} \times\left(\operatorname{OwnDirectPayoff}_{j}^{i}, \text { OwnIndirectPayoff }_{j}^{i},\right. \\
& \left(\text { OthersDirectPayoff }(q)_{j}^{i}\right)_{q \neq i},\left(\operatorname{OthersIndirectPayoff}(q)_{j}^{i}\right)_{q \neq i}, \\
& \text { Delete } \left.\left._{j}, \text { Create }_{j}, \operatorname{Central}_{j}, \text { Isolated }_{j}\right)\right),
\end{aligned}
$$

where Complexity ${ }^{i}$ is the network choice complexity that individual $i$ is facing and $f^{i}$ is a function in which the interaction effects with Complexity ${ }^{i}$ are included.

Structural Complexity First, we examine whether structural complexity in the network formation context affects decision making in a similar way as task complexity in the consumer choice context (e.g., Johnson and Payne 1985). More specifically, we investigate whether changes in structural features like increases in the number of nodes (and thus choice alternatives) in the network, will strengthen individuals' use of heuristics in terms of more strongly focusing on the role of descriptive traits in the utility function and relying more strongly on payoff function simplifications. In our analysis we focus on the feature connected position (i.e., the decision maker is linked 
to the network herself, like "you" in the example of Figure 4.1) as an important aspect of structural complexity, which makes it more difficult for the individual to assess the consequences of changes in the status quo and thus link choice alternatives. Specifically, if an individual is not connected to other agents, her current payoff is trivially equal to zero and link deletion is not possible. As a consequence, she only has to assess the payoffs for link creation with any of the other agents. This brings us to the following hypotheses:

\section{Hypothesis 4 (moderating effects of structural complexity)}

Hypothesis 4.1 Structural complexity increases the impact of the descriptive traits of link choice alternatives (i.e., whether an alternative involves link deletion or creation and whether it concerns an isolated node or a central node) on link choice.

Hypothesis 4.2 Structural complexity decreases the impact of indirect payoff on link choice.

Hypothesis 4.3 Structural complexity decreases the impact of others' payoff on link choice.

Value Transferability Another network feature regarded here is value transferability, which refers to the fact that an individual derives value not only from her direct neighbors, but also indirectly from her neighbors' neighbors, etc. (cf. Section 4.2.2). This network property makes it more complex for individuals to judge the exact value of link choice alternatives, because they have to be forward-looking over indirect links. Value transferability therefore not only enables the heuristic of focusing on the part of the normative value that concerns payoff derived from direct neighbors, but furthermore stimulates the use of the other heuristics. This leads to the following hypotheses: 


\section{Hypothesis 5 (moderating effects of value transferability)}

Hypothesis 5.1 Value transferability increases the impact of the descriptive traits of link choice alternatives (i.e., whether an alternative involves link deletion or creation and whether it concerns an isolated node or a central node) on link choice.

Hypothesis 5.2 Value transferability decreases the impact of others' payoff on link choice.

Social Tradeoff As a final complexity property, we consider social tradeoff, implying that an individual's choices not only affect her own value, but also her neighbors' (and her neighbors' neighbors, etc.) (cf. Section 4.2.2). This network property makes it more complex for individuals with social preferences to judge the exact value of link choice alternatives, because besides their own payoff they have to consider the payoff of (possibly many) other individuals. Therefore, social tradeoff not only enables the heuristic of focusing on the part of the normative value that concerns own payoff, but also has a strengthening effect on the role of the other heuristics. This can be formulated in the following hypotheses:

\section{Hypothesis 6 (moderating effects of social tradeoff)}

Hypothesis 6.1 Social tradeoff increases the impact of the descriptive traits of link choice alternatives (i.e., whether an alternative involves link deletion or creation and whether it concerns an isolated node or a central node) on link choice.

Hypothesis 6.2 Social tradeoff decreases the impact of indirect payoff on link choice.

Our hypotheses are illustrated in Figure 4.2. Note that the heuristics we propose show a positive association with the payoff that individuals normatively should base their decisions on. Therefore, although people do not 
optimize their payoff, they may still optimize their utility by saving effort (cf. Gigerenzer et al. 1999).

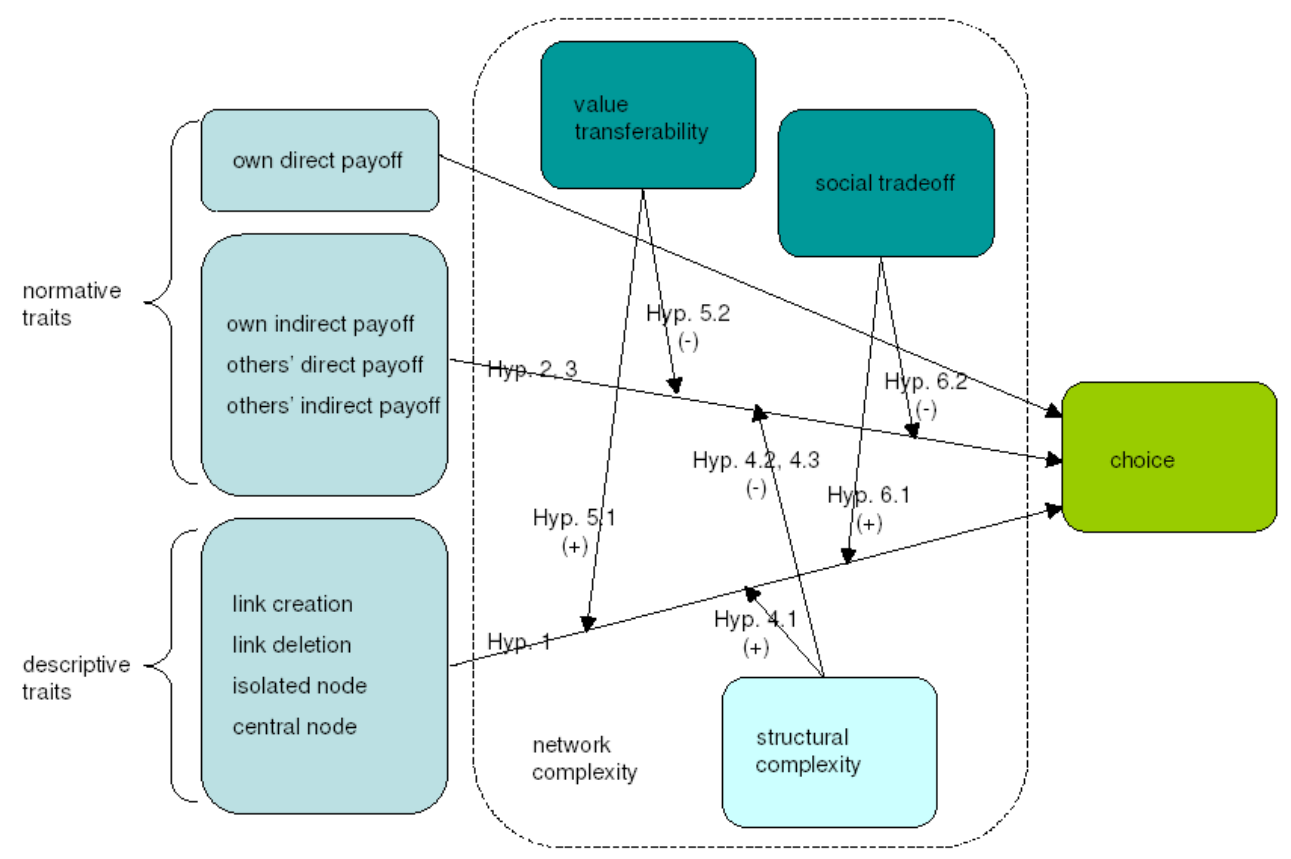

Figure 4.2: Hypothesized Model

\subsection{Methods}

In this section we describe the experimental design as well as the parametric approach used for testing our hypotheses.

\subsubsection{Experimental Design}

Our experiment presented participants with six choice problems similar to that in Figure 4.1. In these problems a participant was allowed to change at most one direct link, i.e., to delete a link that already exists between her and another node, to create a link between her and another node if there is 
not yet one, or to change nothing. The choice problems are illustrated in Tables B.2 and B.3, Appendix B.

To test for the hypothesized shifts in heuristic behavior due to value transferability (equation (4.2) versus equation (4.1)) and social tradeoff (equation (4.3) versus equation (4.1)), we employed four experimental treatments where these two characteristics were the between-subjects factors. Thus, each participant faced one of four particular complexity conditions (see Section 4.2.2). The experimental design is summarized by Table 4.1. A within-subject manipulation for the treatments social and both will be introduced later in this section.

\begin{tabular}{ll|l|l|}
\multicolumn{2}{c}{} & \multicolumn{2}{c}{ social tradeoff? } \\
\cline { 3 - 4 } value & NO & \multicolumn{2}{l}{ YES } \\
\cline { 3 - 4 } transferability? & YES & Yone & social (part 1, part 2) \\
\cline { 3 - 4 } & & transfer & both (part 1, part 2) \\
\cline { 3 - 4 } & &
\end{tabular}

Table 4.1: Experimental Design

Each participant was confronted with a payoff function matching her condition. This function reflects the benefits and costs of link formation and was explained in words to the participants in the instructions (see Appendix A). For a participant $i$ in between-subject treatments none and social there is no value transferability, so value is derived from direct neighbors only. The payoff function is then given by:

$$
\Pi_{i}=\left\{\begin{array}{ccc}
\sum_{j \in N_{i}} \frac{1}{\mu_{i} \cdot \mu_{j}} & \text { if } & \mu_{i}>0 \\
0 & \text { if } & \mu_{i}=0
\end{array}\right.
$$

where $N_{i}$ is the set of nodes with whom $i$ has a direct link, node $j$ is a neighbor of $i$ if $j \in N_{i}$, and $\mu_{i}=\left|N_{i}\right|$ is the number of neighbors of $i$, the degree of $i$.

For between-subject treatments transfer and both there is value transferability, so value is derived from direct as well as indirectly connected nodes. 
The payoff function is then given by:

$$
\Pi_{i}=\left\{\begin{array}{ccc}
\sum_{j \in \bar{N}_{i}} \sum_{p \in \mathcal{P}_{i, j}} \frac{1}{\mu_{i} \cdot \mu_{j} \cdot \prod_{k \in P}\left(\mu_{k}\right)^{2}} & \text { if } & \mu_{i}>0 \\
0 & \text { if } & \mu_{i}=0
\end{array}\right.
$$

where $\bar{N}_{i}$ is the set of nodes with whom $i$ has either a direct or an indirect link, $\mathcal{P}_{i, j}$ is the set of paths between $i$ and $j$, where a path is defined as a sequence of consecutive edges without repeated nodes, $\breve{P}$ is the set of nodes on path $p$ between $i$ and $j$ excluding $i$ and $j$ themselves, and $\mu_{i}$ is the degree of $i$. In the instructions, these payoff functions are not presented in formulas but in easy verbal descriptions, illustrated by an example (see Appendix A).

For between-subject treatments none and transfer there is no social tradeoff. The participants were informed that nobody else was affected by their choices. For treatments social and both there is social tradeoff. The participants were informed that the payoffs for the other nodes in the choice problems are determined analogously to their own payoff, and that the total payoffs created for these other nodes due to their choices are divided equally among the other participants in the room. Thus, a simple form of social preferences, not regarding distributional issues, was evoked. No information or feedback about the tasks and choices of the other participants was provided during the experiment in order to rule out strategic motivations.

Three of the six problems involve a connected position and the other half an isolated position for the participant (see Tables B.2 and B.3, Appendix $\left.\mathrm{B}^{1}\right)$. Thus, the structural complexity characteristic connected position was included as within-subject factor in the experimental design. The number of nodes was kept constant in all six choice problems. Pilot studies conducted by the authors before the experiment indicated that several other structural complexity features like the exact number of links and the number of visual crossings between links do not affect participants' choices. Still, to avoid biases due to such other structural factors, the order of choice problems was rotated among participants.

\footnotetext{
${ }^{1}$ Note that choice problems 1,3 , and 5 involve a connected start position.
} 
To control for individual differences, particularly in social preferences, for participants in between-subject treatments social and both where payoff for other participants had to be considered, an additional part was added to the experiment. This was exactly the same as the first part, but for each choice option the payoff for the participant as well as for the others were mentioned explicitly. This is illustrated in Figure B.1, Appendix B. Charness, Frechette, and Kagel (2004) showed that people behave differently in gift-exchange games when they receive a comprehensive payoff table, and the objective of this additional manipulation is to test in how far participants take others' payoff into account when the complexity of doing so is removed. The payoffs for all choice problems are given in Table B.4 in the same appendix. Thus, for the between-subject treatments social and both, payoff information was also incorporated as a within-subjects factor.

The experiment took place in a computer lab with students and employees of various faculties of Maastricht University, the Netherlands. In each of the four between-subject conditions there were 28 participants in batches of 7. After each choice, feedback was given to the participant about the payoff she earned for herself and if relevant for the other participants in the room, and the maximum and minimum number of points that could have been earned in the specific choice problem. Participants were informed how the payoffs they earn in the experiment are converted into cash euros afterwards, see Sections A.1 and A.2, Appendix A for details. They could only start the experiment after answering control questions correctly and after two really paid-out practice rounds with only three choice alternatives, see Table B.1, Appendix B. At the end they were asked to comment on the way they made their choices in a debriefing part.

\subsubsection{Mixed Logit Estimations}

We perform a comprehensive parametric test of our hypotheses by estimating a mixed (i.e., random parameters) logit model (Hensher et al. 2005). This enables us to establish the roles of several attributes of link alternatives in the network formation process, while allowing for heterogeneity across 
individuals. We assume that (without numerical payoff information), the utility that individual $i$ derives from choosing alternative $j$ in choice problem $t$ is given by:

$$
\begin{aligned}
U_{t j}^{i} & =\sum_{k \in K} \beta_{k}^{i} \cdot P_{k t j}^{i}+\sum_{k \in K} \gamma_{k}^{i} \cdot D_{k t j} \\
& +\sum_{k \in K} \zeta_{k} \cdot C_{t} \cdot P_{k t j}^{i}+\sum_{k \in\{2,3\}} \eta_{k} \cdot C_{t} \cdot D_{k t j} \\
& +\sum_{k \in\{1,2\}} \theta_{k} \cdot S^{i} \cdot P_{k t j}^{i}+\sum_{k \in K} \xi_{k} \cdot S^{i} \cdot D_{k t j} \\
& +\sum_{k \in\{1,3\}} \varphi_{k} \cdot T^{i} \cdot P_{k t j}^{i}+\sum_{k \in K} \chi_{k} \cdot T^{i} \cdot D_{k t j}+\varepsilon_{t j}^{i},
\end{aligned}
$$

where:

$K \quad$ is the set of indices $\{1,2,3,4\}$,

$P_{1 t j}^{i} \quad$ is the own, direct payoff generated for an individual $i$ when in choice problem $t$ she chooses alternative $j$,

$P_{2 t j}^{i} \quad$ is the own, indirect payoff,

$P_{3 t j}^{i} \quad$ is the direct payoff generated for the other participants,

$P_{4 t j}^{i} \quad$ is the indirect payoff generated for the other participants,

$D_{1 t j}$ is a dummy variable indicating link deletion,

$D_{2 t j}$ is a dummy variable indicating link creation,

$D_{3 t j}$ is a dummy variable indicating a central node, i.e., with three or more direct links,

$D_{4 t j}$ is a dummy variable indicating an isolated node, i.e., without direct links,

$C_{t}$ is a dummy variable indicating a connected start position, i.e., not isolated,

$S^{i} \quad$ is a dummy variable indicating social tradeoff, i.e., treatment social or both,

$T^{i} \quad$ is a dummy variable indicating value transferability, i.e., treatment transfer or both, and

$\varepsilon_{t j}^{i} \quad$ is a stochastic variable drawn from a standard Gumble distribution. 
In the random parameter $\beta_{1}^{i}$, superscript $i$ allows for heterogeneity due to individuals' personal preferences as follows:

$$
\beta_{1}^{i}=\beta_{1}+\nu_{1}^{i}
$$

where $\nu_{1}^{i}$ is a stochastic variable drawn from a normal distribution. Analogously, random parameters are included for the other main effects of the payoff and descriptive traits on choice, but in quest of parsimony not for the interaction effects.

Note that in the variables, superscript $i$ allows for heterogeneity due to the between-subject treatment individuals participate in (e.g., in none and social participants do not receive any indirect payoffs and in none and transfer participants do not generate any payoffs for the other participants in the room). Furthermore, notice that interactions between $C$ and $D_{1}, C$ and $D_{4}, S$ and $P_{3}, S$ and $P_{4}, T$ and $P_{2}$, or $T$ and $P_{4}$ do not provide additional information to $D_{1}, D_{4}, P_{3}, P_{4}, P_{2}$, or $P_{4}$, respectively, and therefore are not included.

Then, under the usual assumptions, the unconditional probability that individual $i$ will choose alternative $j$ equals the expected value of the logit probability over all possible values of the random parameters. The model is estimated by Maximum Likelihood with NLOGIT 3.0, Econometric Software, Inc., implementing 500 Halton draws in the Monte Carlo simulation.

Finally, to investigate the impact of providing participants with an explicit payoff overview, which should remove the complexity effects, a similar model is estimated for the data with and without numerical payoff information:

$$
\begin{aligned}
U_{t j}^{i} & =\sum_{k \in K} \beta_{k}^{i} \cdot P_{k t j}^{i}+\sum_{k \in K} \gamma_{k}^{i} \cdot D_{k t j} \\
& +\sum_{k \in K} \zeta_{k} \cdot C_{t} \cdot P_{k t j}^{i}+\sum_{k \in\{2,3\}} \eta_{k} \cdot C_{t} \cdot D_{k t j}
\end{aligned}
$$




$$
\begin{aligned}
& +\sum_{k \in\{1,2\}} \theta_{k} \cdot S^{i} \cdot P_{k t j}^{i}+\sum_{k \in K} \xi_{k} \cdot S^{i} \cdot D_{k t j} \\
& +\sum_{k \in\{1,3\}} \varphi_{k} \cdot T^{i} \cdot P_{k t j}^{i}+\sum_{k \in K} \chi_{k} \cdot T^{i} \cdot D_{k t j} \\
& +\sum_{k \in K} \psi_{1 k} \cdot I_{t}^{i} \cdot P_{k t j}^{i}+\sum_{k \in K} \psi_{2 k} \cdot I_{t}^{i} \cdot D_{k t j} \\
& +\sum_{k \in K} \psi_{3 k} \cdot I_{t}^{i} \cdot C_{t} \cdot P_{k t j}^{i}+\sum_{k \in\{2,3\}} \psi_{4 k} \cdot I_{t}^{i} \cdot C_{t} \cdot D_{k t j} \\
& +\sum_{k \in\{1,3\}} \psi_{5 k} \cdot I_{t}^{i} \cdot T^{i} \cdot P_{k t j}^{i}+\sum_{k \in K} \psi_{6 k} \cdot I_{t}^{i} \cdot T^{i} \cdot D_{k t j}+\varepsilon_{t j}^{i},
\end{aligned}
$$

where $I_{t}^{i}$ is a dummy variable indicating by 1 that participant $i$ was provided with payoff information when solving choice problem $t$. Note that interactions between $I$ and $S \cdot P$ or $I$ and $S \cdot D$ do not provide additional information to $S \cdot P$ or $S \cdot D$, respectively, and therefore are not included.

\subsection{Results}

\subsubsection{Some Illustrative Descriptive Results}

Before turning to a more formal analysis, we first present some illustrative results for the choices made in the different conditions. Hereby the focus is on choice problem 5 (Table $4.2^{2}$ ).

The use of descriptive traits as proposed by Hypothesis 1 can best be illustrated by the results under complexity condition social (second row Table 4.2). Although changing nothing is the status quo, the first option in the list, and the single option that is both normatively optimal for themselves and for the others, only $10.7 \%$ of the respective participants opts for this alternative. However, in the second part of the experiment, when payoff information is given, thus eliminating network complexity, this proportion grows to $53.6 \%$. This corresponds to the use of the descriptive trait link

\footnotetext{
${ }^{2}$ Above this table with choice percentages in the different conditions, the respective choice problem and its payoffs are replicated for the reader's comfort.
} 


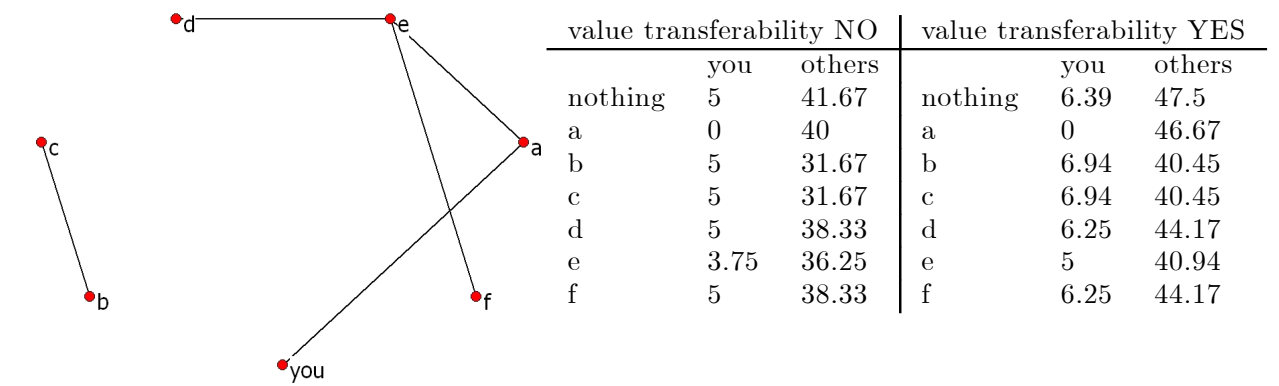

\begin{tabular}{|c|c|c|c|}
\hline \multicolumn{2}{|c|}{ none / payoff info NO } & \multicolumn{2}{|c|}{ transfer / payoff info NO } \\
\hline choice & $\%$ & choice & \\
\hline nothing & 43.3 & nothing & 17.9 \\
\hline $\mathrm{a}$ & 0.0 & a & 0.0 \\
\hline b & 40.0 & b & 60.7 \\
\hline $\mathrm{c}$ & 10.0 & $\mathrm{c}$ & 7.1 \\
\hline $\mathrm{d}$ & 3.3 & $\mathrm{~d}$ & 0.0 \\
\hline $\mathrm{e}$ & 0.0 & $\mathrm{e}$ & 3.6 \\
\hline $\mathrm{f}$ & 3.3 & $\mathrm{f}$ & 10.7 \\
\hline \multicolumn{2}{|c|}{ social / payoff info NO } & \multicolumn{2}{|c|}{ social / payoff info YES } \\
\hline choice & $\%$ & choice & $\%$ \\
\hline nothing & 10.7 & nothing & 53.6 \\
\hline a & 0.0 & a & 0.0 \\
\hline b & 39.3 & $\mathrm{~b}$ & 21.4 \\
\hline $\mathrm{c}$ & 7.1 & $\mathrm{c}$ & 7.1 \\
\hline $\mathrm{d}$ & 17.9 & $\mathrm{~d}$ & 7.1 \\
\hline $\mathrm{e}$ & 0.0 & e & 3.6 \\
\hline $\mathrm{f}$ & 25.0 & $\mathrm{f}$ & 7.1 \\
\hline \multicolumn{2}{|c|}{ both / payoff info NO } & \multicolumn{2}{|c|}{ both / payoff info YES } \\
\hline choice & $\%$ & choice & $\%$ \\
\hline nothing & 35.7 & nothing & 25.0 \\
\hline a & 0.0 & a & 0.0 \\
\hline $\mathrm{b}$ & 28.6 & $\mathrm{~b}$ & 46.4 \\
\hline $\mathrm{c}$ & 14.3 & $\mathrm{c}$ & 25.0 \\
\hline $\mathrm{d}$ & 7.1 & $\mathrm{~d}$ & 3.6 \\
\hline $\mathrm{e}$ & 7.1 & $\mathrm{e}$ & 0.0 \\
\hline $\mathrm{f}$ & 7.1 & $\mathrm{f}$ & 0.0 \\
\hline
\end{tabular}

Table 4.2: Descriptive Results Choice Problem 5

creation as a choice heuristic, where creating a link is qualitatively associated with higher payoff.

In complexity condition none (first row Table 4.2), without value transferability and social tradeoff, all participants choose one of the normatively optimal alternatives, i.e., nothing, b, c, d, or f. However, in condition transfer, where value transferability is included, only $67.8 \%$ of the respective participants choose one of the normatively optimal alternatives, i.e., b or 
c, whereas $96.4 \%$ of the choices would be optimal if only direct payoff is considered. Although causality is undecided, this pattern is in line with Hypothesis 2.

In complexity condition social (second row Table 4.2), where social tradeoff is included, only $10.7 \%$ of the respective participants turns out to opt for the option not to change anything, which reveals social preferences in the sense that it maximizes the payoffs for the other participants, given maximum own payoff. All these participants still maximize their own payoff though. However, in the second part of the experiment, when payoff information is given, thus eliminating network complexity, $53.6 \%$ of the same participants prefers this option. This pattern corresponds with Hypothesis 3.

In complexity condition both (third row Table 4.2), with both value transferability and social tradeoff, only $42.9 \%$ of the respective participants chooses one of the options with optimal own payoff, i.e., b or c, whereas the rest seems to reveal social preferences in the sense that they reduce their own payoff in order to increase others' payoffs. Note that $21.3 \%$ even chooses a Pareto inferior option, i.e., a, d, e, or f, and $92.9 \%$ still maximizes their own, direct payoff. However, in the second part of the experiment, when payoff information is given, thus eliminating network complexity, the proportion with optimal own payoff increases to $71.4 \%$. Also, only $3.6 \%$ chooses a Pareto inferior option. These findings further corroborate Hypotheses 2 and 3.

Further descriptive results, primarily from the debriefing part, are given in Appendix C.

\subsubsection{Mixed Logit Results}

In the following subsections, a comprehensive parametric test of the hypotheses is conducted by estimating a mixed logit model across all experimental conditions for the first part of the experiment without numerical payoff information and then a similar model including interaction effects of such payoff 
information. A p-value of 0.05 is taken as cut-off value for significance.

\section{With Description of Choice Consequences}

Table 4.3 summarizes the estimation results for the first part of the experiment in which participants were asked to make network formation decisions based on a complete description of how the payoffs depend on these choices and no numerical payoff overview was provided.

\begin{tabular}{|c|c|c|c|}
\hline variable & par. & $\begin{array}{r}\text { est. mean } \\
(p-v a l u e)\end{array}$ & $\begin{array}{r}\text { est. st. dev. } \\
\text { (p-value) }\end{array}$ \\
\hline own direct payoff & $\beta_{1}^{2}$ & $.33(.00)$ & $.00(1.0)$ \\
\hline own indirect payoff & $\beta_{2}^{i}$ & $-.16(.53)$ & $.02(.96)$ \\
\hline others' direct payoff & $\beta_{3}^{i}$ & $.02(.68)$ & $.00(.99)$ \\
\hline others' indirect payoff & $\beta_{4}^{i}$ & $-.08(.57)$ & $.00(.98)$ \\
\hline link deletion & $\gamma_{1}^{i}$ & $-1.01(.00)$ & $.00(1.0)$ \\
\hline link creation & $\gamma_{2}^{i}$ & $3.10(.00)$ & $1.79(.00)$ \\
\hline central node & $\gamma_{3}^{i}$ & $-2.30(.00)$ & $1.34(.00)$ \\
\hline isolated node & $\gamma_{4}^{i}$ & $2.65(.00)$ & $.01(.99)$ \\
\hline connected $*$ own direct payoff & $\zeta_{1}$ & $-.43(.00)$ & \\
\hline connected ${ }^{*}$ own indirect payoff & $\zeta_{2}$ & $1.12(.00)$ & \\
\hline connected $*$ others' direct payoff & $\zeta_{3}$ & $.02(.77)$ & \\
\hline connected $*$ others' indirect payoff & $\zeta_{4}$ & $.35(.06)$ & \\
\hline connected $*$ link creation & $\eta_{2}$ & $-3.16(.00)$ & \\
\hline connected $*$ central node & $\eta_{3}$ & $-2.36(.00)$ & \\
\hline social tradeoff $*$ own direct payoff & $\theta_{1}$ & $-.05(.56)$ & \\
\hline social tradeoff $*$ own indirect payoff & $\theta_{2}$ & $.72(.03)$ & \\
\hline social tradeoff $*$ link deletion & $\xi_{1}$ & $-.19(.66)$ & \\
\hline social tradeoff $*$ link creation & $\xi_{2}$ & $.64(.22)$ & \\
\hline social tradeoff $*$ central node & $\xi_{3}$ & $.36(.46)$ & \\
\hline social tradeoff $*$ isolated node & $\xi_{4}$ & $-.26(.62)$ & \\
\hline transferability $*$ own direct payoff & $\varphi_{1}$ & $-.20(.01)$ & \\
\hline transferability $*$ others' direct payoff & $\varphi_{3}$ & $.15(.02)$ & \\
\hline transferability $*$ link deletion & $\chi_{1}$ & $.31(.47)$ & \\
\hline transferability $*$ link creation & $\chi_{2}$ & $-.44(.39)$ & \\
\hline transferability $*$ central node & $\chi_{3}$ & $2.76(.00)$ & \\
\hline transferability $*$ isolated node & $\chi_{4}$ & $-1.17(.03)$ & \\
\hline
\end{tabular}

Table 4.3: Mixed Logit Estimations Without Payoff Information

We find support for the use of descriptive traits additional to the normative payoff in making decisions (Hypothesis 1): link deletion and central node have a significant negative effect on choice and link creation and isolated node a significant positive effect, implying that compared to doing nothing, individuals have an aversion for deleting links and a preference for creating links, and compared to a node with one or two links, individuals 
prefer isolated choice alternatives and dislike nodes with more links, additional to the related payoff consequences.

Moreover, we find support for Hypothesis 2, since the indirect payoff does not significantly influence choice, and preliminary support for Hypothesis 3, since others' payoff does not significantly influence choice.

In case of a connected position for the decision maker and value transferability, the role of the own, direct payoff even significantly decreases, which is in line with Hypotheses 4.1 and 5.1 respectively. However, the complexity conditions do not simply strengthen the basic use of descriptive traits in all cases: under a connected position the tendency to link creation decreases, suggesting that individuals need the feeling of safety from the zero payoff by being connected before being able to optimize their payoff, and under value transferability respondents reverse their preference for isolated nodes and avoidance of central nodes, suggesting that people systematically overestimate value transferability and therefore underestimate relative costs of links. The data do not support Hypothesis 6.1 since under social tradeoff, there is no significant switch from normative to descriptive traits.

Surprisingly, in case of a connected position for the decision maker, there seems to be a switch of focus from direct to indirect payoff, which contradicts Hypothesis 4.2. The role of indirect payoff is also significantly larger in case of social tradeoff, which is the opposite interaction effect from what is predicted by Hypothesis 6.2.

Hypothesis 4.3 is not supported by the data since there is no significant effect of any of the interaction terms of connected position and others' payoff. Hypothesis 5.2 is rejected by the data since the interaction term of value transferability and others' payoff is significantly positive.

Respondents' heuristic behavior significantly varies with respect to the preference for link creation and the avoidance of central nodes. Note that since the own, direct payoff is also included as an explanatory variable in the choice estimation, the found heuristic effects cannot be attributed to the selection of choice problems that participants were presented with. 


\section{With Numerical Payoff Information}

The estimation results for all experimental treatments including the interaction effects of a comprehensive payoff overview are given in Table 4.4.

With this model we find the same support for the use of descriptive traits (Hypotheses 1, 4.1, and 5.1) and the focus on own, direct payoff (Hypotheses 2 and 3) in the case without payoff information. Moreover, we again find the surprising effect of indirect payoff in case of structural complexity of a connected position for the decision maker and in case of social tradeoff, contradicting Hypotheses 4.2 and 6.2. Also the role of other participants' payoff is again significantly larger in case of value transferability, which is the opposite interaction effect from what is predicted by Hypothesis 5.2. Once more we do not find significant results for the effects predicted by Hypotheses 4.3 and 6.1. Lastly, we again find heterogeneity among participants with respect to their preference for link creation and avoidance of central nodes.

More importantly, with this model we find evidence for the role of payoff information indicating that substitution of the exact value of a link choice alternative by more accessible, related heuristic attributes (particular parts of normative traits as well as descriptive traits) is dependent on complexity. Especially, the significant positive effect of others' payoff under payoff information and a connected position for the decision maker confirms that without payoff information, participants are more inclined to be affected by the payoff consequences for other participants than actually reflected in their choices. This completes the proof for Hypothesis 3. Note that $\psi_{13}$ and $\psi_{14}$ are not significant, but for Hypothesis 3 to be supported it is sufficient that $\psi_{33}$ and $\psi_{34}$ show that numerical payoff information makes a difference.

\section{Robustness}

In this section, we check whether our estimation results are robust for several control variables. 


\begin{tabular}{|c|c|c|c|}
\hline variable & par. & est. mean (p) & est. s.d. (p) \\
\hline own direct payoff & $\beta_{1}^{i}$ & $0.33(.00)$ & $0.00(.97)$ \\
\hline own indirect payoff & $\beta_{2}^{i}$ & $-0.14(.57)$ & $0.01(.98)$ \\
\hline others' direct payoff & $\beta_{3}^{i}$ & $0.02(.66)$ & $0.00(.97)$ \\
\hline others' indirect payoff & $\beta_{4}^{i}$ & $-0.08(.58)$ & $0.00(1.0)$ \\
\hline link deletion & $\gamma_{1}^{i}$ & $-1.00(.00)$ & $0.00(.99)$ \\
\hline link creation & $\gamma_{2}^{i}$ & $3.01(.00)$ & $1.73(.00)$ \\
\hline central node & $\gamma_{3}^{i}$ & $-2.39(.00)$ & $1.42(.00)$ \\
\hline isolated node & $\gamma_{4}^{i}$ & $2.61(.00)$ & $0.04(.93)$ \\
\hline connected $*$ own direct payoff & $\zeta_{1}$ & $-0.43(.00)$ & \\
\hline connected $*$ own indirect payoff & $\zeta_{2}$ & $1.11(.00)$ & \\
\hline connected $*$ others' direct payoff & $\zeta_{3}$ & $0.01(.84)$ & \\
\hline connected $*$ others' indirect payoff & $\zeta_{4}$ & $0.34(.07)$ & \\
\hline connected $*$ link creation & $\eta_{2}$ & $-3.02(.00)$ & \\
\hline connected $*$ central node & $\eta_{3}$ & $-2.36(.00)$ & \\
\hline social tradeoff $*$ own direct payoff & $\theta_{1}$ & $-0.04(.62)$ & \\
\hline social tradeoff $*$ own indirect payoff & $\theta_{2}$ & $0.66(.04)$ & \\
\hline social tradeoff $*$ link deletion & $\xi_{1}$ & $-0.19(.66)$ & \\
\hline social tradeoff $*$ link creation & $\xi_{2}$ & $0.60(.23)$ & \\
\hline social tradeoff $*$ central node & $\xi_{3}$ & $0.39(.44)$ & \\
\hline social tradeoff $*$ isolated node & $\xi_{4}$ & $-0.18(.74)$ & \\
\hline transferability $*$ own direct payoff & $\varphi_{1}$ & $-0.19(.01)$ & \\
\hline transferability * others' direct payoff & $\varphi_{3}$ & $0.15(.02)$ & \\
\hline transferability $*$ link deletion & $\chi_{1}$ & $0.30(.48)$ & \\
\hline transferability $*$ link creation & $\chi_{2}$ & $-0.54(.28)$ & \\
\hline transferability $*$ central node & $\chi_{3}$ & $2.81(.00)$ & \\
\hline transferability $*$ isolated node & $\chi_{4}$ & $-1.10(.04)$ & \\
\hline payoff info $*$ own direct payoff & $\psi_{11}$ & $0.31(.04)$ & \\
\hline payoff info $*$ own indirect payoff & $\psi_{12}$ & $1.65(.03)$ & \\
\hline payoff info $*$ others' direct payoff & $\psi_{13}$ & $0.01(.91)$ & \\
\hline payoff info $*$ others' indirect payoff & $\psi_{14}$ & $-0.03(.91)$ & \\
\hline payoff info $*$ link deletion & $\psi_{21}$ & $-1.00(.15)$ & \\
\hline payoff info $*$ link creation & $\psi_{22}$ & $0.20(.87)$ & \\
\hline payoff info $*$ central node & $\psi_{23}$ & $-1.21(.24)$ & \\
\hline payoff info $*$ isolated node & $\psi_{24}$ & $-1.54(.04)$ & \\
\hline payoff info $*$ connected $*$ own direct payoff & $\psi_{31}$ & $-0.41(.02)$ & \\
\hline payoff info $*$ connected $*$ own indirect payoff & $\psi_{32}$ & $-1.49(.05)$ & \\
\hline payoff info $*$ connected $*$ others' direct payoff & $\psi_{33}$ & $0.19(.04)$ & \\
\hline payoff info $*$ connected $*$ others' indirect payoff & $\psi_{34}$ & $0.72(.02)$ & \\
\hline payoff info $*$ connected $*$ link creation & $\psi_{42}$ & $-1.34(.30)$ & \\
\hline payoff info $*$ connected $*$ central node & $\psi_{43}$ & $3.12(.04)$ & \\
\hline payoff info $*$ transferability $*$ own direct payoff & $\psi_{51}$ & $-0.26(.23)$ & \\
\hline payoff info $*$ transferability $*$ others' direct payoff & $\psi_{53}$ & $0.10(.45)$ & \\
\hline payoff info $*$ transferability $*$ link deletion & $\psi_{61}$ & $-0.17(.87)$ & \\
\hline payoff info $*$ transferability $*$ link creation & $\psi_{62}$ & $3.19(.00)$ & \\
\hline payoff info $*$ transferability $*$ central node & $\psi_{63}$ & $-2.99(.03)$ & \\
\hline payoff info $*$ transferability $*$ isolated node & $\psi_{64}$ & $-1.11(.33)$ & \\
\hline
\end{tabular}

Table 4.4: Mixed Logit Estimations Including Payoff Information

Learning Effects First of all, the main model for all between-subject treatments without payoff information is re-estimated including the control dummy variable experience, which tracks whether the respective participant 
at the moment of choice was solving either one of her last three problems or one of her first three, as an additional interaction. We find that the behavioral effects do not change and that the overall model fit does not improve, but the interactions of experience and some descriptive traits turn out to be significant. The respective signs of the parameter estimations are such that the use of these heuristics is stronger for more experienced individuals, so the behavioral effects in network formation decisions as explored in the current chapter are not transitory.

Order Effects Next, the main model for all between-subject treatments without payoff information is re-estimated including the control dummy variable primacy, which tracks whether the respective alternative was listed either as one of the first four or as one of the last four choice options, as an additional choice predictor. Again we find that the behavioral effects remain and that the overall model fit hardly improves, whereas the respective parameter estimate is positive and significant.

Random Error Effects Finally, we compare our model to a simpler, more restricted model based on the prior predictions as described in Section 4.2.1 without our behavioral effects. In line with Charness and Jackson (2006), we add a random component to the individual decision strategy. The variance of this random component is allowed to vary between complexity conditions. Thus, we assume that without payoff information, the utility that individual $i$ derives from choosing alternative $j$ in choice problem $t$ is given by:

$$
U_{t j}^{i}=\beta_{1}^{i} \cdot\left(P_{1 t j}^{i}+P_{2 t j}^{i}\right)+\beta_{2}^{i} \cdot\left(P_{3 t j}^{i}+P_{4 t j}^{i}\right)+\varepsilon_{t j}^{i},
$$

where the random parameter $\beta_{1}^{i}$ is allowed to linearly depend on the complexity conditions $C_{t}, S^{i}$, and $T^{i}$. This rival model turns out to perform worse in terms of model fit (i.e., the Akaike Information Criterion increases from 3.47 to 3.84 ), strengthening our claim of systematic, heuristic effects of complexity on link choice behavior. 


\subsection{Discussion}

\subsubsection{Results}

This study shows that network complexity influences individual link choice behavior in a systematic way, since it affects individuals' use of heuristics in which the normative target attribute of a link choice alternative (i.e., payoff for themselves and possibly for other individuals) is assessed by substituting more accessible properties of that alternative. These heuristic attributes can either be parts of the normative traits: the payoff from their direct neighbors (Hypothesis 2) and their own payoff (Hypothesis 3), or descriptive traits: whether a link choice alternative involves link deletion or creation and whether it concerns an isolated or a central node (Hypothesis 1). Specifically, individuals tend to neglect indirect and others' payoff whereas they do significantly consider their own, direct payoff, they have an aversion for deleting links and a preference for creating links, and they prefer isolated choice alternatives and dislike nodes with more than two direct links, additional to the related payoff consequences. Furthermore, we find that complexity of one type can moderate individuals' dealing with a complex feature of another type. In particular, facing the structural complexity of a connected position (Hypothesis 4.1) or facing value transferability (Hypothesis 5.1), individuals tend to rely more strongly on descriptive traits. A summary of the results is provided in Table 4.5.

\begin{tabular}{|l|l|}
\hline Hypothesis & Result \\
\hline behavioral effects on network formation & \\
1: use of descriptive traits & supported \\
2: focus on direct payoff & supported \\
3: focus on own payoff & supported \\
\hline moderating effects of network complexity & \\
4: structural complexity & partially supported \\
5: value transferability & partially supported \\
6: social tradeoff & not supported \\
\hline
\end{tabular}

Table 4.5: Summary Experimental Results 
An explanation for the lack of support of the hypotheses related to social tradeoff is suggested by participants' replies to the debriefing questions. It seems that individuals consciously decide to completely neglect any payoff they generate for other people as soon as they are confronted with social tradeoff in a complex network setting. Therefore, a connected position and value transferability are not able to still strengthen their tendency to focus on own payoff (Hypotheses 4.3 and 5.2), and it is not necessary for them to also use other heuristics to deal with the added complexity of social tradeoff (Hypotheses 6.1 and 6.2).

The opposite effects for Hypotheses 4.2, 5.2, and 6.2 are more difficult to account for. It might be that individuals need a trigger to realize that it makes sense to consider payoff for others, namely that value is transferred over links (Hypothesis 5.2). Vice versa, the fact that their choices also influence other participants' payoff might be a trigger to make individuals realize that indirect payoff is also relevant (Hypothesis 6.2). With respect to Hypothesis 4.2, it might be that individuals need the feeling of safety from the zero payoff by being connected before being able to consider indirect payoff as to optimize their payoff.

The model estimations with inclusion of payoff information confirm that individuals' heuristic focus on the own, direct part of the normative trait and consideration of descriptive traits is based on the complexity of the network setting. This is also illustrated by the descriptive statistics presented in Section 4.4.1: the same individuals make significantly different decisions in the part of the experiment with simplifying payoff information than in the part without. In particular, this finishes the proof of Hypothesis 3, since apparently not all insignificant effects of payoff for others on choice are simply due to a lack of social preferences.

\subsubsection{Implications}

The current research pioneers the issue of behavioral effects in individual decisions of network formation. Our results should raise interest in future 
research into this realm, for they have important implications for experimental research as well as application areas of network formation.

For instance, experimental research practice is often disposed to make the payoff consequences of choices as transparent as possible for participants as to prevent biased findings due to their wrong understanding of the instructions. However, we claim that this modifies participants' behavior in a systematic way, since it eliminates complexity that they otherwise would handle by using heuristics. As we have shown in Section 4.4.2 (page 107), these changes in behavior cannot accurately be captured by models not explicitly allowing for behavioral effects, even if these models allow for variations in unobserved error according to network complexity.

Furthermore, in one of the applications of network formation as mentioned in the introduction, word-of-mouth communication among consumers, it matters for the supplier of the respective product or service to be aware of behavioral effects as found in this study. For instance, consumers have a tendency to talk with isolated peers and they neglect communication benefits from beyond their direct neighbors in their communication decisions. Also, she can exploit the finding that this heuristic behavior is dependent on the complexity of the network environment.

One of the directions that follow-up studies could take concerns the question in how far the complexity types and behavioral effects we considered are specific for the network context. For example, would the tendency to link creation have an equivalent in other choice settings? Moreover, future experiments could generate further insights in the linking choice process of individuals by concentrating on specific effects from the rich range of heuristic tendencies explored here. 


\section{Chapter 5}

\section{Conclusion}

This chapter concludes on the dissertation's aim (Section 5.1) and suggests managerial implications (Section 5.2) and directions for further research (Section 5.3).

\subsection{OCCNs: An Economic Exploration of their Formation and Value}

The topic of Online Consumer-to-Consumer Communication Networks was studied in this dissertation, because the importance of consumers is increasingly recognized (C), a prevailing way in which consumers achieve this is by communicating with other consumers $(\mathrm{C})$, human behavior is affected by the entire pattern of relationships among actors $(\mathrm{N})$, and online communication is growingly popular $(\mathrm{O})$.

Communication in OCCNs is prominently characterized by informational and social benefits as well as time and effort costs for the participants. Therefore, as illustrated in Figure 1.1, we took an economic perspective on the underlying network formation process as a determinant of value. The focus was on the strategic aspect, where participants choose to create links based on a cost-benefit tradeoff when the effects of these choices also depend 
on others' choices, as well as on the behavioral aspect, where the complexity of the network setting can influence the mental decision rules that individual participants use for link formation.

In Chapter 2, we studied the strategic aspect by introducing a gametheoretic model of OCCN formation incorporating the mentioned characteristic benefits and costs. We distinguished between social and informational value as motivations for bilateral exchange decisions, where informational value can be transferred relatively easily to third parties through indirect links, whereas social value is more individual and therefore not transferable. Moreover, we introduced link specificity in the communication context, meaning that the more direct connections an individual has to maintain with other individuals, the less she is able to specify her attention per link within her total time available. Therefore, her additive value per link for others declines and she also derives less additive value from each link with others.

For homogeneous participants and a fixed level of specificity, the model predicts which network structures emerge dependent on the level of focus on informational versus social value in the community. We first characterized the set of stable structures in the case with only social value to indicate the separate impact of link specificity and demonstrate that it includes a wide range of non-standard architectures. Then in the case with both social and informational value, the joint effect of link specificity and value transferability was shown to reduce the stable set to fragmented architectures consisting of small star components. Finally, the best and worst stable structures from a welfare point of view were described and the role of the relative community focus on informational versus social value was illustrated.

Chapter 3 generalized this model as to allow for heterogeneous participants and a variable level of specificity. By simulations, we showed which networks emerge for three levels of link specificity, three levels of focus on informational versus social value from communication, and four valuation patterns: homogeneity and expertise heterogeneity, each with and without the presence of one especially highly valued expert. It was illustrated that 
the structure properties of these networks heavily depend on these parameter values, which results in efficiency and equity differences among settings. For example, we found that the effects of an expert in a community depend on whether the rest of the community is homogeneous or not. Also, when the informational and social aspects of communication are equally valued, more focus on informational value turned out to be beneficial for community welfare. Finally, enhancing communication in the community by decreasing link specificity does not always lead to higher welfare from communication.

In Chapter 4, we investigated the individual link choice process in its behavioral aspect using experimental economics. It was demonstrated that individuals systematically simplify more complex components of network payoff in their linking decisions. Specifically, they focus on only part of the normative payoff, namely on their own, direct payoff, and tend to ignore indirect payoff and payoff for others in the network. Additionally, individuals use descriptive behavioral traits of link choice alternatives to guide their choices: they are sensitive to whether an alternative involves link deletion or creation and whether it concerns an isolated or a central node. Furthermore, we found that complexity of one type can moderate individuals' dealing with a complex feature of another type.

\subsection{Managerial Implications}

Our game-theoretical approach of the strategic aspect in OCCN formation provides a framework for assessing the effectivity of several managerial tools to affect the value that members derive from communication and therefore indirectly also the firm's welfare. The parameters of interest as studied are the informational versus social orientation in the OCCN, the community's valuation pattern with in particular the presence or absence of an expert, and the level of link specificity in the OCCN.

The local efficiency results of Section 3.5 illustrated that in several parameter settings, social preferences in the sense that members use community 
welfare as their objective function rather than their own payoff can enhance welfare or equity to a consequential extent. Moreover, the experiments discussed in Chapter 4 demonstrated that people often are willing to take others' payoff into account when making network formation decisions if only it is clear what the payoff consequences of these decisions are. Therefore, providing more insight into this is an alternative area managers can think of in trying to affect the welfare and equity in their community.

Also, the behavioral effects in individual decisions of OCCN formation that we demonstrated by the use of experimental economics have important implications for managers working in this area. For example, it matters for a supplier of the product or service related to the OCCN to be aware of consumers' tendency to talk with isolated peers and to neglect communication benefits from beyond their direct neighbors in their communication decisions. Also, a manager can exploit the finding that this heuristic behavior is dependent on the complexity of the network environment.

\subsection{Further Research}

\subsubsection{The Strategic Aspect}

With respect to the game-theoretical approach of the strategic aspect of OCCN formation, it is possible to derive more general analytical results also for parameter settings that we currently have covered by simulation studies only.

In addition, follow-up studies can investigate the effects of an agent who is given incentives by the community manager to serve welfare rather than own payoff. Preliminary simulations indicate that this will lead to more efficient pairwise stable networks, even if this special agent is not an expert in the sense that other community members derive more value from communication with her.

Furthermore, more complex forms of valuation heterogeneity can be ex- 
plored. For example, the informational and social valuations could be systematically varied, resulting in different effects of the informational versus social orientation in the OCCN. Also, besides expertise heterogeneity as studied here - agents represent different values for their fellow customers it is possible to study judgement heterogeneity, where agents have different opinions on the values of their fellow customers (e.g., Galeotti et al. 2006). Preliminary simulations indicate that this leads to agent permutations rather than structure differences.

Another extension of the current model could be to relax the assumption that agents divide their available effort equally among all their relationships, thus dropping the common 1-0 formulation of links to recognize the notion of strong and weak ties (e.g., Constant, Sproull, and Kiesler 1996).

The managerial implication practice of affecting parameters of the model might bring up other ways of controlling the welfare and equity of OCCNs asking for further research. Empirical testing forms an alternative promising area: the applicability of the used payoff function with diverse parameter settings could be examined, also the strategic model could be tested experimentally, contributing to an emerging literature as surveyed by Kosfeld (2004), and finally, the model could be orderly confronted with the rich availability of OCCN data (e.g., Fisher et al. 2006, Holme et al. 2004).

\subsubsection{The Behavioral Aspect}

With respect to the experimental approach of the behavioral aspect of OCCN formation, a general implication holds for experimental research practice, which is often disposed to make the payoff consequences of choices as transparent as possible for participants as to prevent biased findings due to their wrong understanding of the instructions. However, we claim that this modifies participants' behavior in a systematic way, since it eliminates complexity that they otherwise would handle by using heuristics. As we have shown in Section 4.4.2, these changes in behavior cannot accurately be captured by models not explicitly allowing for behavioral effects, even if 
these models allow for variations in unobserved error according to network complexity.

One of the directions that follow-up studies could take concerns the question in how far the complexity types and behavioral effects we considered are specific for the network context. For example, would the tendency to link creation have an equivalent in other choice settings? Moreover, future experiments could generate further insights in the linking choice process of individuals by concentrating on specific effects from the rich range of heuristic tendencies explored in Chapter 4.

Revisiting Figure 1.1 (page 8), there is one dashed arrow suggesting an interesting further research direction. We found in the behavioral aspect of OCCN formation that individuals systematically simplify more complex components of network payoff in their linking decisions. It could be investigated how this in turn affects the strategic OCCN formation process and therefore the value that participants (and managers) derive from communication.

Consequently, we hope that this dissertation stimulates future research in the appealing area of OCCNs and moreover illustrated the usefulness of an economic approach of their formation and value. 


\section{Appendix A}

\section{Instructions}

\section{A.1 Social Tradeoff? NO Value Transferabi- lity? NO YES}

In this experiment you are asked to respond to eight choice problems. You can earn points depending on the choices you make in these problems. The total number of points that you have at the end of the experiment determines your monetary payoff.

In each problem, you see a picture of a network in which you and several other nodes are interconnected by links. In order to generate points, you are allowed to change at most one link. You have the following options to do this: (1) you can delete one link that already exists between you and any other node, (2) you can create one link between you and any other node if there is not yet any link between you and this node, or (3) you can choose not to change anything.

You can determine the number of points you receive due to your choice for a specific problem, as follows. For each node you are directly linked with (we call such a node a neighbor) you obtain points. For each path that links you to some other node you obtain points. However, there 
is also some cost associated with being connected: the number of points you receive for each of your direct neighbors equals 10 divided by two components: (i) the number of direct neighbors you have, and (ii) the number of direct neighbors this neighbor has. the number of points you receive for each path that links you to some other node equals 10 divided by three components: (i) the number of direct neighbors you have in the network, (ii) the number of direct neighbors this other node has in the network, and (iii) the square of the number of direct neighbors that any of the further nodes on the path between you and the other node has in the network.

[example, see Section A.3]

After each of the eight problems, the number of points that you earned will be reported.

Note that there are no real people behind the other nodes in a network: you are the only one able to change a link and earn points by this.

At the end of the experiment, points are exchanged for euros in the following way: amount in euros you receive $=4+\mathbf{0 . 4} 0.3$ (total number of points that you earned - $\mathbf{3 3 . 6 8} 45.17$ ).

\section{A.2 Social Tradeoff? YES Value Transferabi- lity? NO YES}

In this first part you are asked to respond to eight choice problems. You can earn points depending on the choices you make in these problems. Moreover, your choices can also generate points for the other participants in the room. The total number of points that you have at the end of the experiment determines your monetary payoff.

In each problem, you see a picture of a network in which you and several other nodes are interconnected by links. In order to generate points, you are allowed to change at most one link. You have the following options to 
do this: (1) you can delete one link that already exists between you and any other node, (2) you can create one link between you and any other node if there is not yet any link between you and this node, or (3) you can choose not to change anything.

You can determine the number of points you receive due to your choice for a specific problem, as follows. For each node you are directly linked with (we call such a node a neighbor) you obtain points. For each path that links you to some other node you obtain points. However, there is also some cost associated with being connected: the number of points you receive for each of your direct neighbors equals 10 divided by two components: (i) the number of direct neighbors you have, and (ii) the number of direct neighbors this neighbor has. the number of points you receive for each path that links you to some other node equals 10 divided by three components: (i) the number of direct neighbors you have in the network, (ii) the number of direct neighbors this other node has in the network, and (iii) the square of the number of direct neighbors that any of the further nodes on the path between you and the other node has in the network.

[example, see Section A.3]

After each of the eight problems, the number of points that you earned will be reported.

The other nodes in the choice problems receive points in the same way as you do. There are no real people behind these nodes and you are the only one able to change a link in a network. However, the points that the other nodes receive due to your choices do have a consequence for the other participants in this room. In fact, these points will be divided equally among them.

[example continued, see Section A.3]

The number of points that you generated for the other participants will also be reported after each problem. 
At the end of the experiment, points are exchanged for euros in the following way: amount in euros you receive for this first part $=4+\mathbf{0 . 0 6}$ 0.07 (total number of points that you earned in this first part - $\mathbf{2 6 5 . 5 1}$ 320.63).

The choices you made in the first part do not influence the payoffs in this part and the choices you will make in this part do not influence the payoffs in the previous part.

In this second part you are asked to respond to eight choice problems. You can earn points depending on the choices you make in these problems. Moreover, your choices can also generate points for the other participants in the room. The total number of points that you have at the end of the experiment determines your monetary payoff.

At the end of the experiment, points are exchanged for euros in the following way: amount in euros you receive for this second part $=0.5+$ 0.030 .035 (total number of points that you earned in this second part $\mathbf{2 6 5 . 5 1} 320.63)$.

\section{A.3 Example}

- Social Tradeoff? NO Value Transferability? NO

For example, in the above network [Figure 4.1, page 85] you have two direct neighbors: a and d. For neighbor a you get 10 points divided by 2 (since you have two direct neighbors) divided by 3 (since a has three direct neighbors). For neighbor d you get 10 points divided by 2 (since you have two direct neighbors) divided by 2 (since $\mathrm{d}$ has two direct neighbors). In total you therefore receive $10 / 6+10 / 4=25 / 6$ points in this example.

- Social Tradeoff? NO Value Transferability? YES

For example, in the above network [Figure 4.1] there are two paths between you and c. For the path via a and b you get 10 points divided 
by 2 (since you have two direct neighbors in the network) divided by 3 * 3 (since a has three direct neighbors in the network) divided by $2 *$ 2 (since $\mathrm{b}$ has two direct neighbors in the network) divided by 1 (since $\mathrm{c}$ has one direct neighbor in the network). For the path via d, a, and b you get 10 points divided by 2 (since you have two direct neighbors in the network) divided by $2 * 2$ (since $\mathrm{d}$ has two direct neighbors in the network) divided by $3 * 3$ (since a has three direct neighbors in the network) divided by $2^{*} 2$ (since $\mathrm{b}$ has two direct neighbors in the network) divided by 1 (since $\mathrm{c}$ has one direct neighbor in the network). In total you therefore receive $10 / 72+10 / 288=25 / 144$ points for the paths between you and c. In the same way you get $10 / 36+10 / 144$ points for the paths between you and b, 10/6 + 10/24 points for the paths between you and a and $10 / 4+10 / 36$ points for the paths between you and $d$. In total you therefore receive 775/144 points in this example.

\section{- Social Tradeoff? YES Value Transferability? NO}

For example, in the above network [Figure 4.1] you have two direct neighbors: a and d. For neighbor a you get 10 points divided by 2 (since you have two direct neighbors) divided by 3 (since a has three direct neighbors). For neighbor d you get 10 points divided by 2 (since you have two direct neighbors) divided by 2 (since $\mathrm{d}$ has two direct neighbors). In total you therefore receive $10 / 6+10 / 4=25 / 6$ points in this example.

[continued] In the example above, node c has one direct neighbor: b. Therefore, she receives 10 points divided by 1 (since $\mathrm{c}$ has one direct neighbor) divided by 2 (since $\mathrm{b}$ has two direct neighbors), which implies 5 points. In the same way, node b gets $10 / 2+10 / 6=20 / 3$ points, node a gets $10 / 6+10 / 6+10 / 6=5$ points, and node $\mathrm{d}$ gets $10 / 4+10 / 6=25 / 6$ points. In total therefore $5+20 / 3+5+25 / 6$ $=125 / 6$ points will be divided equally among the other participants in the room in this example. 
- Social Tradeoff? YES Value Transferability? YES

For example, in the above network [Figure 4.1] there are two paths between you and c. For the path via a and b you get 10 points divided by 2 (since you have two direct neighbors in the network) divided by 3 * 3 (since a has three direct neighbors in the network) divided by $2 *$ 2 (since $\mathrm{b}$ has two direct neighbors in the network) divided by 1 (since $\mathrm{c}$ has one direct neighbor in the network). For the path via d, a, and b you get 10 points divided by 2 (since you have two direct neighbors in the network) divided by $2 * 2$ (since $\mathrm{d}$ has two direct neighbors in the network) divided by $3 * 3$ (since a has three direct neighbors in the network) divided by $2 * 2$ (since $\mathrm{b}$ has two direct neighbors in the network) divided by 1 (since $\mathrm{c}$ has one direct neighbor in the network). In total you therefore receive $10 / 72+10 / 288=25 / 144$ points for the paths between you and c. In the same way you get $10 / 36+10 / 144$ points for the paths between you and b, 10/6+10/24 points for the paths between you and a and 10/4 +10/36 points for the paths between you and d. In total you therefore receive $775 / 144$ points in this example.

[continued] In the example above, there is one path between nodes a and c. Therefore, c receives 10 points divided by 1 (since $\mathrm{c}$ has one direct neighbor) divided by $2 * 2$ (since $\mathrm{b}$ has two direct neighbors) divided by 3 (since a has three direct neighbors) $=5 / 6$ points for the paths between her and a. In the same way c gets $10 / 2$ points for the path between her and $\mathrm{b}, 10 / 72+10 / 288$ points for the paths between her and $\mathrm{d}$, and 10/72 $+10 / 288$ points for the paths between her and you. In total c therefore receives $445 / 72$ points. In the same way node b gets $10 / 2+10 / 6+10 / 36+10 / 144+10 / 36+10 / 144$ points, node a gets $10 / 12+10 / 6+10 / 6+10 / 24+10 / 6+10 / 24$ points, and node d gets $10 / 72+10 / 288+10 / 36+10 / 144+10 / 6+10 / 24+10 / 4+$ $10 / 36$ points. In total therefore $445 / 72+265 / 6+20 / 3+775 / 144=$ $2995 / 48$ points will be divided equally among the other participants in the room in this example. 


\section{Appendix B}

\section{Choice Problems}

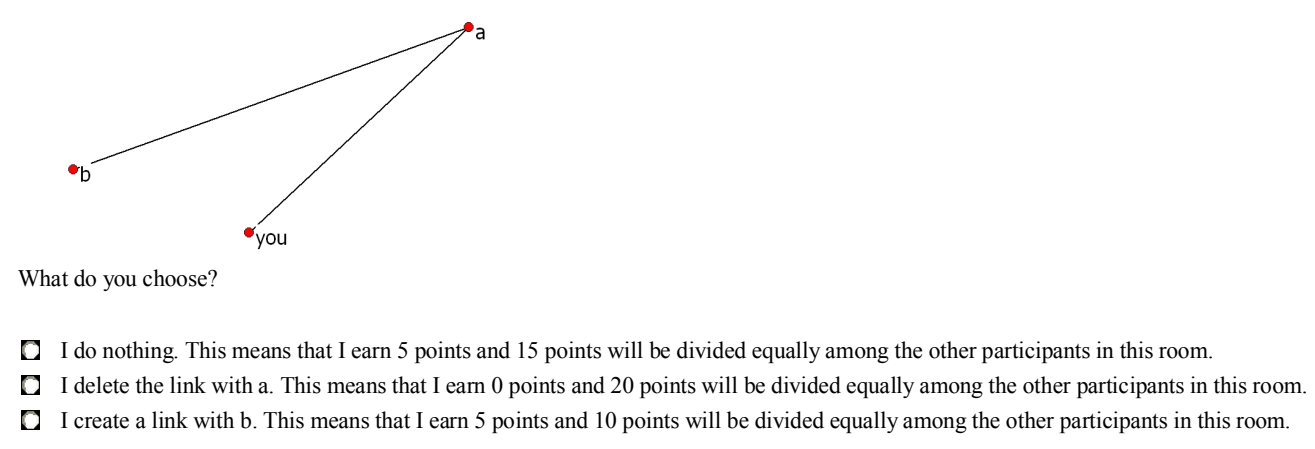

Figure B.1: Illustration Payoff Information 


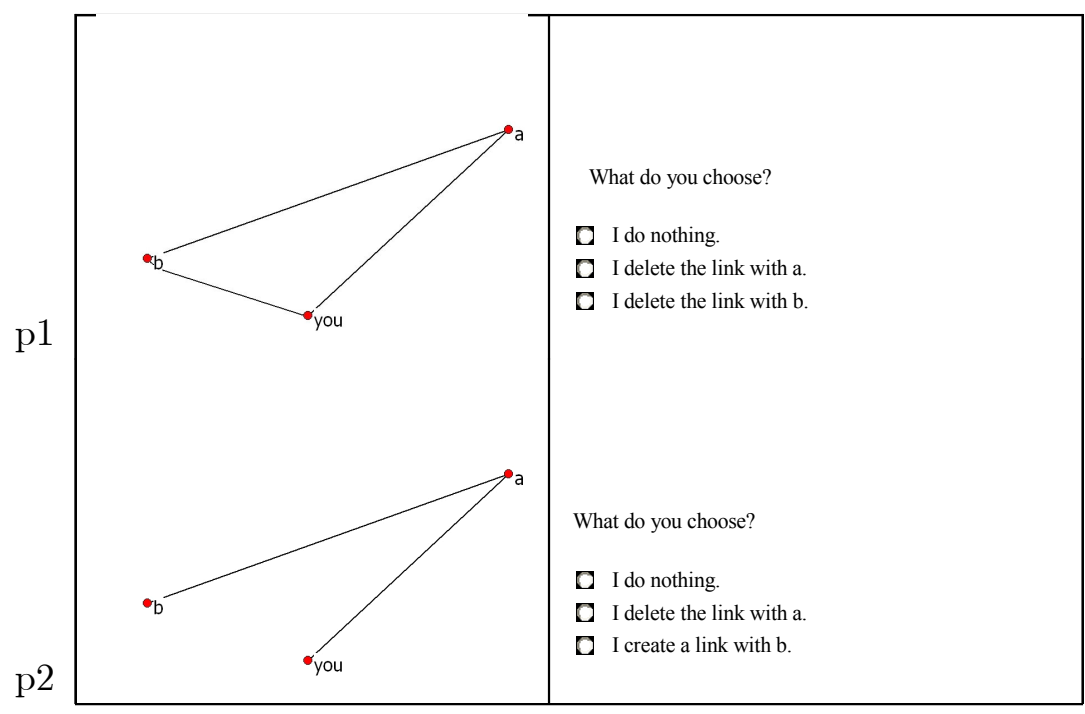

Table B.1: Practice Rounds 


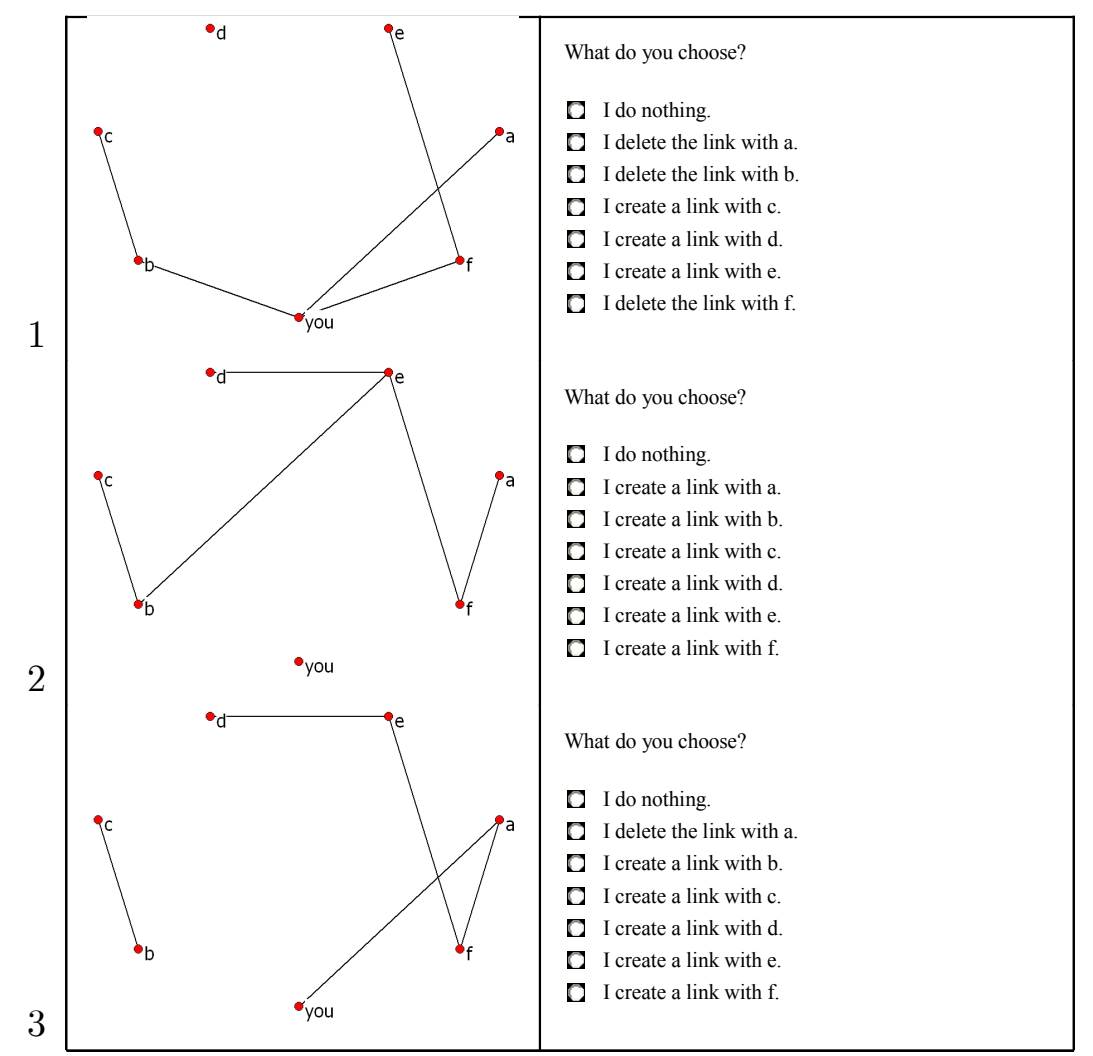

Table B.2: Choice Problems 1 - 3 


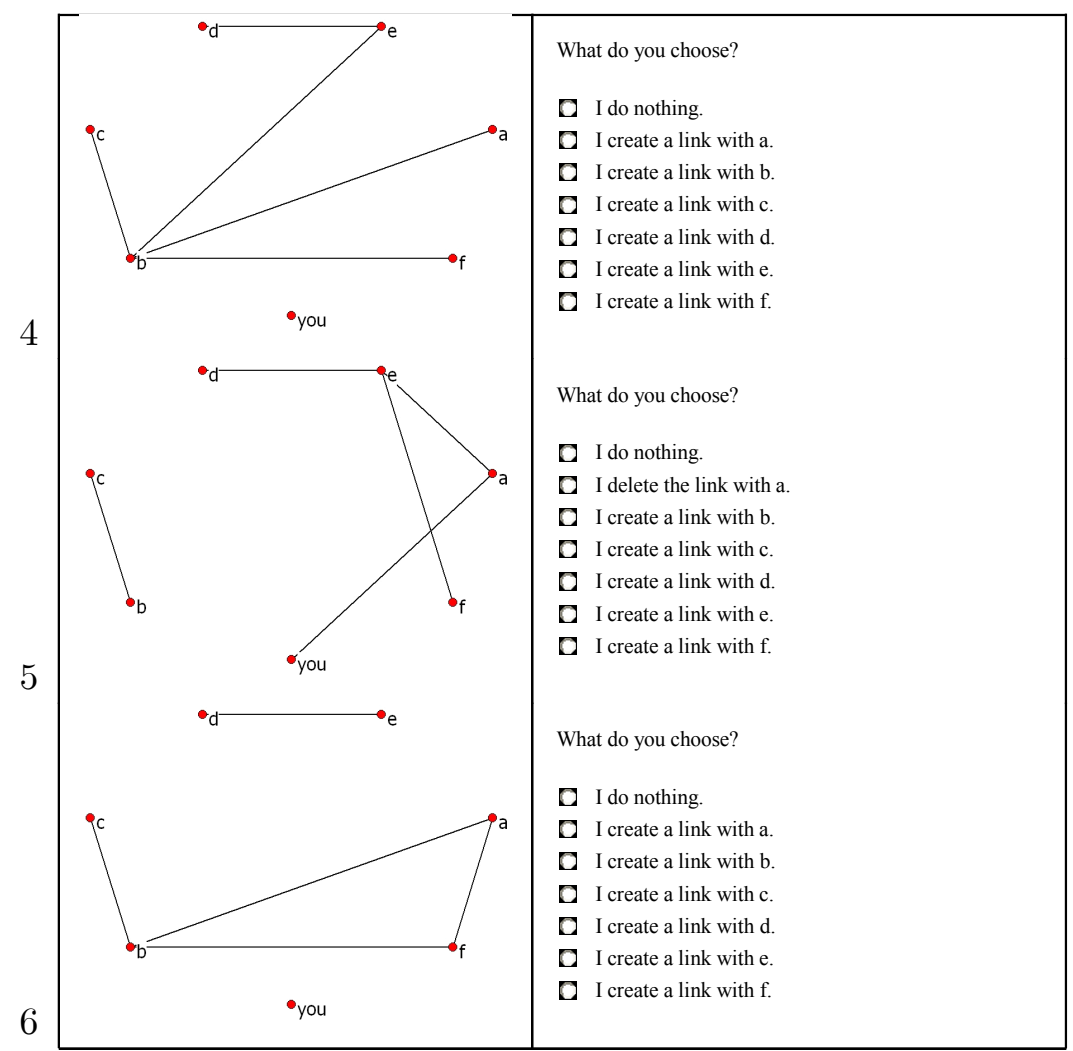

Table B.3: Choice Problems 4 - 6 


\begin{tabular}{|c|c|c|c|c|c|c|}
\hline \multirow{2}{*}{1} & \multicolumn{3}{|c|}{ indirect payoffs: NO } & \multicolumn{3}{|c|}{ indirect payoffs: YES } \\
\hline & & you & others & & you & others \\
\hline & nothing & 6.67 & 26.67 & nothing & 8.33 & 32.92 \\
\hline & a & 5 & 25 & $\mathrm{a}$ & 7.5 & 30.31 \\
\hline & b & 7.5 & 37.5 & b & 8.75 & 42.5 \\
\hline & $\mathrm{c}$ & 6.25 & 21.25 & $\mathrm{c}$ & 7.5 & 26.48 \\
\hline & d & 7.5 & 27.5 & d & 8.75 & 34.45 \\
\hline & e & 6.25 & 21.25 & $\mathrm{e}$ & 7.5 & 26.48 \\
\hline & $\mathrm{f}$ & 7.5 & 37.5 & $\mathrm{f}$ & 8.75 & 42.5 \\
\hline \multirow[t]{8}{*}{2} & & you & others & & you & others \\
\hline & nothing & 0 & 33.33 & nothing & 0 & 41.25 \\
\hline & $\mathrm{a}$ & 5 & 33.33 & $\mathrm{a}$ & 6.58 & 41.51 \\
\hline & b & 3.33 & 32.22 & b & 5.03 & 39.72 \\
\hline & $\mathrm{c}$ & 5 & 33.33 & $\mathrm{c}$ & 6.58 & 41.51 \\
\hline & d & 5 & 35 & d & 6.25 & 42.5 \\
\hline & e & 2.5 & 32.5 & $\mathrm{e}$ & 4.06 & 39.14 \\
\hline & $\mathrm{f}$ & 3.33 & 32.22 & $\mathrm{f}$ & 5.03 & 39.72 \\
\hline \multirow[t]{8}{*}{3} & & you & others & & you & others \\
\hline & nothing & 5 & 45 & nothing & 6.72 & 51.09 \\
\hline & $\mathrm{a}$ & 0 & 45 & $\mathrm{a}$ & 0 & 51.25 \\
\hline & b & 5 & 35 & b & 7.11 & 44 \\
\hline & $\mathrm{c}$ & 5 & 35 & $\mathrm{c}$ & 7.11 & 44 \\
\hline & d & 5 & 40 & d & 6.64 & 46.56 \\
\hline & e & 4.17 & 39.17 & $\mathrm{e}$ & 5.83 & 44.58 \\
\hline & $\mathrm{f}$ & 4.17 & 40.83 & $\mathrm{f}$ & 5.38 & 45.59 \\
\hline \multirow[t]{8}{*}{4} & & you & others & & you & others \\
\hline & nothing & 0 & 27.5 & nothing & 0 & 35.31 \\
\hline & a & 5 & 30 & a & 6.05 & 37.15 \\
\hline & b & 2 & 26 & b & 3.5 & 32.7 \\
\hline & $\mathrm{c}$ & 5 & 30 & $\mathrm{c}$ & 6.05 & 37.15 \\
\hline & d & 5 & 27.5 & d & 6.52 & 35.74 \\
\hline & e & 3.33 & 26.67 & $\mathrm{e}$ & 4.93 & 34.24 \\
\hline & $\mathrm{f}$ & 5 & 30 & $\mathrm{f}$ & 6.05 & 37.15 \\
\hline \multirow[t]{8}{*}{5} & & you & others & & you & others \\
\hline & nothing & 5 & 41.67 & nothing & 6.39 & 47.5 \\
\hline & a & 0 & 40 & $\mathrm{a}$ & 0 & 46.67 \\
\hline & b & 5 & 31.67 & b & 6.94 & 40.45 \\
\hline & $\mathrm{c}$ & 5 & 31.67 & $\mathrm{c}$ & 6.94 & 40.45 \\
\hline & d & 5 & 38.33 & d & 6.25 & 44.17 \\
\hline & e & 3.75 & 36.25 & $\mathrm{e}$ & 5 & 40.94 \\
\hline & $\mathrm{f}$ & 5 & 38.33 & $\mathrm{f}$ & 6.25 & 44.17 \\
\hline \multirow[t]{8}{*}{6} & & you & others & & you & others \\
\hline & nothing & 0 & 38.33 & nothing & 0 & 43.33 \\
\hline & $\mathrm{a}$ & 3.33 & 38.89 & a & 4.57 & 43.58 \\
\hline & b & 2.5 & 37.5 & $\mathrm{~b}$ & 3.91 & 42.03 \\
\hline & $\mathrm{c}$ & 5 & 40 & $\mathrm{c}$ & 6.18 & 44.79 \\
\hline & d & 5 & 33.33 & d & 7.5 & 40.83 \\
\hline & $\mathrm{e}$ & 5 & 33.33 & e & 7.5 & 40.83 \\
\hline & $\mathrm{f}$ & 3.33 & 38.89 & $\mathrm{f}$ & 4.57 & 43.58 \\
\hline
\end{tabular}

Table B.4: Payoffs Choice Problems 


\section{Appendix C}

\section{Descriptive Results}

1. Duration: average 40,2 min., stand. dev. 14,8 min.

2. Almost all participants tried to earn as much as possible, whereas 17 subjects indicated other goals: best choices (6), fun / interest (2), optimal own payoffs and not too bad payoffs for the others (4), optimal own payoffs and minimal payoffs for the others (1), structural goals (4).

3. In the first choice problem [practice round], participants chose as follows: at random: 1 , by calculation: 60 , by intuition: 34 , using a rule: 13, namely connect to the one with the least neighbors / shortest paths (13), otherwise: 6, namely mix of intuition and calculation (5), mistake in understanding instructions at first (1).

4. Thereafter, did participants change their strategies? No: 67 , for the strategy was good or convenient and the problems were similar, yes: 47, switch (more) to calculation (12), intuition / experience (22), or rule mentioned in descriptive 3 (11), or consider other participants more (2).

5. In conditions social and both, did participants take into account the points created for other participants? 36 did not, since they didn't think about it (4), didn't care about it (16), didn't know how (5) or didn't like the effort (11), 20 did, where they (conditionally) maximized $(>=8)$ or minimized $(>=3)$ the points for the others, $>=2$ participants 
seem not to understand that dividing among other participants does not include yourself.

6. Strategies in the second part of conditions social and both: (conditionally) maximizing payoffs for the others (25), choosing not too badly for the others (7), (conditionally) minimizing payoffs for the others (6), trying to repeat part 1 (8), unclear (10).

7. Strategic considerations in conditions social and both? No: 18, since they didn't think about it (7), thought that the other participants wouldn't care (5), the other participants are outside control (4), or it would be too difficult (2), yes, but did not influence choices: 9, yes, hoping for a favorable group: 5, or expecting an unfavorable group: 2, yes, unclear how: 22 ( $>=5$ of these seem not to understand that this question is about the others creating points for you and not about you creating points for the others).

8. Difficulties were in the following fields: calculation: 33 , choice complexity: 34, instructions: 27, equivalent options: 5, none: 16 .

9. Further remarks: interesting / nice: 12, want to know more about the experiment: 10, confirming what was said before: 5, suggestions: 10 .

10. Age: average 22,5 yrs., stand. dev.: 3,4 yrs.

11. Male: 48 , female: 66 .

12. Dutch: 40, German: 43, Chinese: 9, other: 22.

13. Faculty of Economics \& Business Administration: 90, other: 24.

14. 90 participants did not participate in a similar experiment before; 24 did.

15. 112 participants would like to participate in future experiments, 2 would not.

16. In conditions social and both: 40 participants did not know any of their fellow session participants, 12 knew one and 4 knew more. 


\section{Bibliography}

Achrol, R.S. and P. Kotler (1999), "Marketing in the Network Economy," Journal of Marketing 63(4), 146-163.

Algesheimer, R., U.M. Dholakia, and A. Herrmann (2005), "The Social Influence of Brand Community: Evidence from European Car Clubs," Journal of Marketing 69(3), 19-34.

Alstyne, M. van and E. Brynjolfsson (2005), "Global Village or CyberBalkans? Modeling and Measuring the Integration of Electronic Communities," Management Science 51(6), 851-868.

Asvanund, A., K. Clay, R. Krishnan, and M.D. Smith (2004), "An Empirical Analysis of Network Externalities in Peer-to-Peer Music-Sharing Networks," Information Systems Research 15(2), 155-174.

Bagozzi, R.P. and U.M. Dholakia (2006), "Antecedents and Purchase Consequences of Customer Participation in Small Group Brand Communities," International Journal of Research in Marketing 23(1), 45-61.

Bala, V. and S. Goyal (2000), "A Noncooperative Model of Network Formation," Econometrica 68(5), 1181-1229.

Belleflamme, P. and F. Bloch (2004), "Market Sharing Agreements and Collusive Networks," International Economic Review 45(2), 387-411. 
Berninghaus, S.K., K.-M. Ehrhart, and M. Ott (2006), "A Network Experiment in Continuous Time: The Influence of Link Costs", Experimental Economics 9(3), 237-251.

Bonner, S.E. (1994), "A Model of the Effects of Audit Task Complexity", Accounting, Organizations and Society 19(3), 213-234.

Broniarczyk, S.M. and J.W. Alba (1994), "Theory versus Data in Prediction and Correlation Tasks", Organizational Behavior and Human Decision Processes 57(1), 117-139.

Brown, M., A. Falk, and E. Fehr (2004), "Relational Contracts and the Nature of Market Interactions", Econometrica 72(3), 747-780.

Callander, S. and C.R. Plott (2005), "Principles of Network Development and Evolution: An Experimental Study", Journal of Public Economics 89(8), 1469-1495.

Calvó-Armengol, A. and Y. Zenou (2004), "Social Networks and Crime Decisions: the Role of Social Structure in Facilitating Delinquent Behavior," International Economic Review 45(3), 939-958.

Camerer, C. (1998), "Bounded Rationality in Individual Decision Making", Experimental Economics 1(2), 163-183.

Charness, G., G.R. Frechette, and J.H. Kagel (2004), "How Robust is Laboratory Gift Exchange?", Experimental Economics 7(2), 189-205.

Charness, G. and M.O. Jackson (2006), "Group Play in Games and the Role of Consent in Network Formation", forthcoming in Journal of Economic Theory.

Constant, D., L. Sproull, and S. Kiesler (1996), "The Kindness of Strangers: The Usefulness of Electronic Weak Ties for Technical Advice", Organization Science 7(2), 119-135.

Corbae, D. and J. Duffy (2007), "Experiments with Network Formation", forthcoming in Games and Economic Behavior. 
Currarini, S. (2007), "Network Design in Games with Spillovers," Review of Economic Design 10(4), 305-326.

Deck, C. and C. Johnson (2004), "Link Bidding in Laboratory Networks", Review of Economic Design 8(4), 359-372.

Dellarocas, C. (2003), "The Digitization of Word of Mouth: Promise and Challenges of Online Feedback Mechanisms," Management Science 49(10), 1407-1424.

Dholakia, U.M., R.P. Bagozzi, and L. Klein Pearo (2004), "A Social Influence Model of Consumer Participation in Network- and Small-GroupBased Virtual Communities," International Journal of Research in Marketing 21(3), 241-263.

Dodds, P.S., R. Muhamad, and J.W. Watts (2003), "An Experimental Study of Search in Global Social Networks," Science 301(5634), 827-829.

Fehr, E. and K.M. Schmidt (2003), "Theories of Fairness and Reciprocity Evidence and Economic Applications", in: Dewatripont, M., L.P. Hansen, and S. Turnovsky, Advances in Economics and Econometrics: Theory and Applications, Eighth World Congress of the Econometric Society 1, Cambridge: University Press, 208-257.

Fisher, D., M. Smith, and H.T. Welser (2006), "You Are Who You Talk To: Detecting Roles in Usenet Newsgroups," Proceedings of the 39th Hawaii International Conference on System Sciences 3, 59.2.

Forbus, K.D. (1993), "Qualitative Process Theory: Twelve Years After", Artificial Intelligence 59(1-2), 115-123.

Frederick, S., G. Loewenstein, and T. O'Donoghue (2002), "Time Discounting and Time Preference: A Critical Review", Journal of Economic Literature 40(2), 351-401.

Galeotti, A., S. Goyal, and J. Kamphorst (2006), "Network Formation with Heterogeneous Players," Games and Economic Behavior 54(2), 353-372. 
Gigerenzer, G., P.M. Todd and the ABC Research Group (1999), Simple Heuristics That Make Us Smart, Oxford: University Press.

Gilovich, T., D. Griffin, and D. Kahneman (2002), Heuristics and Biases: The Psychology of Intuitive Judgment, Cambridge: University Press.

Goyal, S. (2005), "Strong and Weak Links," Journal of the European Economic Association 3(2-3), 608-616.

Goyal, S. and S. Joshi (2003), "Networks of Collaboration in Oligopoly," Games and Economic Behavior 43(1), 57-85.

Goyal, S. and F. Vega-Redondo (2007), "Structural Holes in Social Networks," forthcoming in Journal of Economic Theory.

Granovetter, M. (1995), Getting a Job: A Study of Contacts and Careers, 2nd ed., Chicago and London: University of Chicago Press.

Granovetter, M. (2005), "The Impact of Social Structure on Economic Outcomes," Journal of Economic Perspectives 19(1), 33-50.

Hagel III, J. and A.G. Armstrong (1997), Net Gain: Expanding Markets Through Virtual Communities, Boston MA: HBS Press.

Hanaki, N., A. Peterhansl, and P.S. Dodds (2007), "Cooperation in Evolving Social Networks", Management Science 53(7), 1036-1050.

Hannon, B. (1994), "Sense of Place: Geographic Discounting by People, Animals and Plants", Ecological Economics 10(2), 157-174.

Hauk, E. and R. Nagel (2001), "Choice of Partners in Multiple Two-Person Prisoner's Dilemma Games", Journal of Conflict Resolution 45(6), 770793.

Hennig-Thurau, T., K.P. Gwinner, G. Walsh, and D.D. Gremler (2004), "Electronic Word-Of-Mouth via Consumer-Opinion Platforms: What Motivates Consumers to Articulate Themselves on the Internet?, Journal of Interactive Marketing 18(1), 38-52. 
Hensher, D.A., J.M. Rose, and W.H. Greene (2005), Applied Choice Analysis: A Primer, Cambridge: University Press.

Holme, P., C.R. Edling, and F. Liljeros (2004), "Structure and Time Evolution of an Internet Dating Community," Social Networks 26(2), 155-174.

Iacobucci, D. and N. Hopkins (1992), "Modeling Dyadic Interactions and Networks in Marketing", Journal of Marketing Research 29(1), 5-17.

Iacobucci, D. (ed.) (1996), Networks in Marketing, Thousand Oaks, London, and New Delhi: SAGE Publications.

Jackson, M.O. and A. Van den Nouweland (2005), "Strongly Stable Networks", Games and Economic Behavior 51(2), 420-444.

Jackson, M.O. and A. Wolinsky (1996), "A Strategic Model of Social and Economic Networks," Journal of Economic Theory 71(1), 44-74.

Jap, S.D. (1999), "Pie-Expansion Efforts: Collaboration Processes in BuyerSupplier Relationships", Journal of Marketing Research 36(4), 461-475.

Johnson, E.J. and J.W. Payne (1985), "Effort and Accuracy in Choice", Management Science 31(4), 395-414.

Kahneman, D. (2003), "Maps of Bounded Rationality: Psychology for Behavioral Economics", The American Economic Review 93(5), 1449-1475.

Kirchsteiger, G., M. Niederle, and J. Potters (2005), "Endogenizing Market Institutions: An Experimental Approach", European Economic Review 49(7), 1827-1853.

Kosfeld, M. (2004), "Economic Networks in the Laboratory: A Survey," Review of Network Economics 3(1), 20-41.

Kranton, R.E. and D.F. Minehart (2001), "A Theory of Buyer-Seller Networks", American Economic Review 91(3), 485-508.

Levinthal, D.A. and J.G. March (1993), "The Myopia of Learning”, Strategic Management Journal 14(special issue winter), 95-112. 
Loasby, B.J. (1976), Choice, complexity and ignorance, Cambridge: University Press.

McFadden, D. (2001), "Economic Choices", The American Economic Review 91(3), 351-378.

McKelvey, R.D. and T.R. Palfrey (1995), "Quantal Response Equilibria for Normal Form Games", Games and Economic Behavior 10(1), 6-38.

McLure Wasko, M. and S. Faraj (2005), "Why Should I Share? Examining Social Capital and Knowledge Contribution in Electronic Networks of Practice," MIS Quarterly 29(1), 35-57.

Perrings, C. and B. Hannon (2001), "An Introduction to Spatial Discounting", Journal of Regional Science 41(1), 23-38.

Prahalad, C.K. and V. Ramaswamy (2000), "Co-opting Customer Competence", Harvard Business Review 78(1), 79-87.

Rheingold, H. (2000), The Virtual Community: Homesteading on the Electronic Frontier, London: MIT Press.

Robbins, L. (1932), An Essay on the Nature and Significance of Economic Science, London: Macmillan.

Shafir, E., I. Simonson, and A. Tversky (1993), "Reason-Based Choice", Cognition 49(1-2), 11-36.

Simon, H.A. (1979), "Rational Decision Making in Business Organizations", The American Economic Review 69(4), 493-513.

Swait, J. and W. Adamowicz (2001), "The Influence of Task Complexity on Consumer Choice: A Latent Class Model of Decision Strategy Switching", Journal of Consumer Research 28(1), 135-148.

Van den Bulte, C. and R.K. Moenaert (1998), "The Effects of R\&D Team Co-location on Communication Patterns among R\&D, Marketing, and Manufacturing", Management Science 44(11), S1-S18. 
Von Hippel, E. (1998), "Economics of Product Development by Users: The Impact of 'Sticky' Local Information”, Management Science 44(5), 629-644. Wasserman, S. and K. Faust (1994), Social Network Analysis: Methods and Applications, Cambridge: University Press.

Wellman, B. and S.D. Berkowitz (eds.) (1988), Social Structures: A Network Approach, Cambridge: University Press.

Wellman, B., J. Salaff, D. Dimitrova, L. Garton, M. Gulia, and C. Haythornthwaite (1996), "Computer Networks as Social Networks: Collaborative Work, Telework, and Virtual Community," Annual Review of Sociology 22(1), 213-238.

Wiertz, C. (2005), The Kindness of Strangers: Studies on Customer Behavior in Commercial Virtual Communities, Maastricht: University Press. 


\section{Nederlandse Samenvatting (Summary in Dutch)}

\section{Communicatienetwerken onder consumenten via het internet: een economisch onderzoek naar hun vorming en waarde}

Het belang van consumenten wordt tegenwoordig steeds meer erkend. Tijdens de afgelopen eeuw ontwikkelde de heersende bedrijfsfilosofie zich van het productie-idee, dat consumenten ziet als vanzelfsprekende kopers van het product van een bedrijf, via het verkoopidee, dat consumenten ziet als mogelijke kopers van het product van een bedrijf die overtuigd moeten worden, naar het marketingidee, dat consumenten ziet als individuen met behoeften waaraan het product van een bedrijf moet voldoen. Nog recenter worden consumenten zelfs vaak gezien als actieve partners in het productieproces van een bedrijf, aangezien zij de specialisten zijn van hun eigen voorkeuren en - na aankoop - van het gebruiken van het product.

Een veelvoorkomende manier waarop consumenten deze actieve rol kunnen vervullen is door met andere consumenten te communiceren. Door ervaringen met een product uit te wisselen, helpen consumenten niet alleen zichzelf en elkaar, maar tegelijkertijd helpen ze het bedrijf als dat voldoende kennisgeoriënteerd en flexibel is. Het spreekt vanzelf dat consumenten door elkaar te helpen de ondersteuningskosten van het bedrijf reduceren. Daarnaast voorzien ze het bedrijf van insiderfeedback die nuttig is voor productverbetering en ontwikkelen en bevorderen ze een positievere houding 
ten opzichte van het product. Welvaart van consument en bedrijf zijn in deze context dus sterk gerelateerd.

In de sociale wetenschappen en in marketing in het bijzonder wordt erkend dat menselijk gedrag bestudeerd moet worden vanuit een structuurof netwerkperspectief. Dit omdat gedrag niet alleen beïnvloed wordt door individuele zaken, bijvoorbeeld bedrijf, consument of huishouden, of zelfs paren, bijvoorbeeld koper-verkoper of consument-consument, maar door het gehele patroon van relaties onder meerdere spelers. Daarom behandelen we in dit proefschrift het relevante onderwerp van onderlinge consumentencommunicatie in de vorm van netwerken.

Een steeds populairdere versie van zo'n netwerk is het online consumerto-consumer communication network (OCCN). Via het internet zijn consumenten vrijer om hun communicatiepartners te kiezen op basis van gedeelde interesse dan in de gewone wereld, want ze worden minder gehinderd door bestaande kennissenstructuren. Ook is het veel eenvoudiger voor een bedrijf om inzicht in en zelfs invloed op de communicatie onder consumenten te verkrijgen, wat nodig is om de voordelen ervan optimaal te kunnen benutten. Enkele diverse voorbeelden van OCCN's worden geïllustreerd op de omslag van dit boek.

Communicatie in OCCN's wordt opvallend gekenmerkt door het feit dat het twee verschillende categorieën van waarde en daardoor motivatie aan deelnemers levert: informatieve waarde, die gebaseerd is op de uitwisseling van productgerelateerde kennis, en sociale waarde, die gebaseerd is op het genoegen van onderling contact. Een ander prominent kenmerk van OCCN's, tot nu toe grotendeels genegeerd door de wetenschappelijke literatuur, is het feit dat er ook kosten bij komen kijken voor deelnemers, want ze moeten tijd en moeite besteden aan het onderhouden van hun communicatieverbindingen met andere leden: in de eerste helft van 2008 besteedde een gemiddelde consument $37 \%$ van haar tijd op het internet aan communicatie, terwijl de andere categorieën handel, inhoud en zoeken betreffen.

Sinds haar recente ontstaan heeft de marketingliteratuur over dit onder- 
werp zich voornamelijk toegespitst op de vraag waarom individuen ervoor kiezen deel te nemen en bij te dragen aan gemeenschappen op het internet en kregen tot nu toe de bijzonderheden van deze virtuele netwerken van persoonlijke relaties geen aandacht. Toch kunnen structuren van wie met wie communiceert wel degelijk empirisch vastgesteld worden en hebben ze invloed op uitkomsten zoals de mate waarin waarde over het netwerk verspreid wordt en hoe die waarde onderling verdeeld wordt. Dit zijn belangrijke variabelen aangezien ze bepalen hoe gunstig OCCN's zijn voor zowel consumenten als bedrijven.

De wetenschappelijke discipline economie bestudeert menselijke keuzen met betrekking tot schaarse zaken als middelen om doelen te bereiken. Zoals eerder vermeld is onderlinge consumentencommunicatie kostbaar (schaarste) en haar structuur is in belangrijke mate bepalend voor haar waarde (doel), dus keuzen van consumenten met betrekking tot het vormen van een communicatienetwerk passen perfect in het economische vakgebied. Aangezien marketing een multidisciplinaire wetenschap is die haar theorieën over consumentengedrag baseert op sociologie, antropologie, filosofie, psychologie en economie, is het opvallend dat de economische benadering van OCCNvorming klaarblijkelijk ontbreekt in de bestaande marketingliteratuur.

Het huidige proefschrift heeft als doel dit gat te dichten door een economisch onderzoek naar de vorming en waarde van OCCN's te beginnen. Hiervoor spitsen we ons toe op twee verschillende, maar sterk onderling verweven aspecten: (i) het strategische aspect, waarbij deelnemers ervoor kiezen op basis van een afweging van kosten en baten verbindingen te maken wanneer de gevolgen hiervan mede afhangen van de keuzen van anderen, en (ii) het gedragsaspect, waarbij de complexiteit van de netwerkomgeving van invloed kan zijn op de mentale beslisregels die individuele deelnemers gebruiken bij hun verbindingskeuzen.

Om het netwerkvormingsproces in haar strategische aspect te modelleren gebruiken we het economische gereedschap bij uitstek om strategische interacties te bestuderen, namelijk de speltheorie. Deze subdiscipline beoogt situaties waarin besluitvormers hun keuzen vrij kunnen baseren op de kosten 
en baten van de alternatieven, die mede afhankelijk zijn van de keuzen van andere besluitvormers, wiskundig te vatten. Recentelijk ontwikkelt zich een speltheoretische literatuurstroom die zich toespitst op netwerkvorming, opgevat als een proces waarin individuen ervoor kiezen verbindingen te leggen en te onderhouden als de deelnemers aan de verbinding daar baat bij hebben, wat resulteert in een stabiele netwerkstructuur. Hoewel deze modellen zeer geschikt zijn om toe te passen op een internetomgeving, is dit nog niet eerder gedaan.

Voor deze toepassing nemen we de genoemde kosten- en batengerelateerde kenmerken van OCCN's op in ons speltheoretische model van netwerkvorming. We maken als eersten onderscheid tussen sociale en informatieve waarde als motivatie voor bilaterale uitwisselingsbeslissingen, waarbij informatieve waarde relatief eenvoudig via indirecte verbindingen aan derden kan worden doorgegeven, terwijl sociale waarde individueler is en daarom niet doorgeefbaar. Bovendien introduceren we verbindingsspecificiteit in de communicatiecontext, wat inhoudt dat hoe meer directe connecties met anderen een individu moet onderhouden, hoe minder ze in staat is haar aandacht per verbinding te specificeren binnen de haar beschikbare tijd. Daarom neemt haar toegevoegde waarde per verbinding voor anderen af en ontleent ze ook zelf minder toegevoegde waarde aan elke verbinding met anderen.

Ons speltheoretische model wordt gepresenteerd in Hoofdstuk 2 van dit proefschrift. Voor homogene deelnemers en een vaste mate van verbindingsspecificiteit voorspelt het model welke netwerkstructuren ontstaan afhankelijk van de mate van concentratie op informatieve versus sociale waarde in de gemeenschap. Om de afzonderlijke gevolgen van verbindingsspecificiteit aan te geven, karakteriseren we eerst de verzameling stabiele structuren voor het geval met alleen sociale waarde en tonen aan dat die een grote verscheidenheid aan niet-standaard architecturen omvat. Vervolgens laten we zien dat in het geval met zowel sociale als informatieve waarde, het gezamenlijke effect van verbindingsspecificiteit en doorgeefbaarheid van waarde de stabiele verzameling reduceert tot gefragmenteerde architecturen die uit kleine stervormige componenten bestaan. Tenslotte worden de sta- 
biele structuren omschreven die het beste en slechtste zijn vanuit een welvaartsperspectief en wordt de rol van de relatieve gemeenschapsconcentratie op informatieve versus sociale waarde geïllustreerd.

In Hoofdstuk 3 wordt het model gegeneraliseerd zodat het heterogene deelnemers en een variabele mate van verbindingsspecificiteit toestaat. Aan de hand van simulaties laten we zien welke netwerken ontstaan bij drie niveaus van verbindingsspecificiteit, drie niveaus van concentratie op informatieve versus sociale waarde van communicatie en vier onderlinge waarderingspatronen: homogeniteit en expertise-heterogeniteit, elk met en zonder de aanwezigheid van een bijzonder hooggewaardeerde expert. Er wordt getoond dat de structuureigenschappen van deze netwerken sterk afhangen van deze parameterwaarden, wat resulteert in efficiëntie- en gelijkmatigheidverschillen tussen situaties. Hiermee beoordelen we de effectiviteit van verscheidene managementgereedschappen voor OCCN's.

Om het individuele verbindingskeuzeproces in haar gedragsaspect te onderzoeken, gebruiken we een benadering vanuit de experimentele economie. Deze tak onderzoekt keuzen door ze mensen in een gecontroleerde laboratoriumomgeving te laten maken, waarbij de gevolgen van deze keuzen in geld aan de deelnemers uitbetaald worden. Er bestaat recente en groeiende literatuur waarin wetenschappers het netwerkvormingsproces experimenteel onderzoeken. Deze studies houden zich allemaal bezig met het strategische aspect van netwerkvorming, terwijl wij dit aspect uitsluiten om het gedragsaspect beter te kunnen bestuderen, aangezien de effecten van beide aspecten onderling verward kunnen zijn.

Om onze vraag hoe de complexiteit van de netwerkomgeving de mentale beslisregels kan beïnvloeden die deelnemers bij verbindingsvorming gebruiken te beantwoorden, combineren we de experimenteel-economische benadering met een modellering van individuele keuzen zoals bekend van de discipline keuzeanalyse. Hierdoor kunnen we de gevolgen van belangrijke netwerkeigenschappen op beslissingen onderzoeken zonder sterke aannamen te hoeven maken over de strategische wisselwerking tussen heterogene, foutgevoelige besluitvormers. 
Hoofdstuk 4 beschrijft ons experiment met haar resultaten en toont aan dat individuen gecompliceerdere onderdelen van netwerkuitbetaling systematisch vereenvoudigen in hun verbindingsbeslissingen. In het bijzonder concentreren ze zich slechts op een gedeelte van de normatieve uitbetaling, namelijk op hun eigen, directe uitbetaling, en neigen naar het verwaarlozen van indirecte uitbetaling en uitbetaling voor anderen in het netwerk. Daarnaast gebruiken individuen beschrijvende gedragskenmerken van alternatieven om hun verbindingskeuzen op te baseren: ze zijn er gevoelig voor of een alternatief gaat om het verbreken of juist vormen van een verbinding en of het een geïsoleerd of een centraal iemand betreft. Verder ontdekken we dat complexiteit van een bepaald type de manier waarop individuen omgaan met een complexe eigenschap van een ander type beïnvloedt. Deze gedragseffecten hebben belangrijke implicaties voor onderzoekers en managers die te maken hebben met netwerkvorming. 


\section{Curriculum Vitae}

Marjolein J.W. Harmsen - van Hout was born on April 29th 1979 in Ravenstein, The Netherlands. After completing a Gymnasium-bèta education at the St.-Janscollege in Hoensbroek (Heerlen) in 1997, she studied Econometrics (specializations Econometrics and Mathematical Economics) and Economics (specialization International Competition Policy) at the Faculty of Economics and Business Administration of Maastricht University and graduated for both on April 29th 2003. Her final thesis was titled "Networks: Formation Models and Efficiency Issues". From May 1st 2003 Marjolein worked on her PhD project about Consumer Communication Networks at the Department of Marketing of the same university and was supervised by prof. dr. ir. Benedict G.C. Dellaert (Erasmus University Rotterdam) and prof. dr. P. Jean-Jacques Herings (Department of Economics). Since October 27th 2008 she has been proceeding as a post-doctoral researcher with the institute of Future Energy Consumer Needs and Behavior at the Faculty of Business and Economics and the E.ON Energy Research Center of RWTH Aachen University, Germany. Marjolein is married and lives in Simpelveld, The Netherlands. 